NOTE TO USERS

This reproduction is the best copy available. 



\title{
ROLE OF BLADE-ON-PETIOLE1 AND 2 IN PATTERNING THE ARABIDOPSIS THALIANA LEAF AND INFLORESCENCE
}

by

Mingli Xu

\begin{abstract}
A thesis submitted to
The Faculty of Graduate Studies and Postdoctoral Affairs

in partial fulfillment of the requirements for the degree of
\end{abstract}

Doctor of Philosophy

in

Biology

Department of Biology

Carleton University

Ottawa, Ontario, Canada

August, 2011

(C)2011, Mingli Xu 
Library and Archives

Canada

Published Heritage

Branch

395 Wellington Street

Ottawa ON K1A ON4

Canada
Bibliothèque et

Archives Canada

Direction du

Patrimoine de l'édition

395 , rue Wellington

Ottawa ON K1A ON4

Canada
Your file Votre référence
ISBN: $978-0-494-83236-3$
Our file Notre reférence
ISBN: $978-0-494-83236-3$
NOTICE:

The author has granted a nonexclusive license allowing Library and Archives Canada to reproduce, publish, archive, preserve, conserve, communicate to the public by telecommunication or on the Internet, loan, distribute and sell theses worldwide, for commercial or noncommercial purposes, in microform, paper, electronic and/or any other formats.

The author retains copyright ownership and moral rights in this thesis. Neither the thesis nor substantial extracts from it may be printed or otherwise reproduced without the author's permission.
AVIS:

L'auteur a accordé une licence non exclusive permettant à la Bibliothèque et Archives Canada de reproduire, publier, archiver, sauvegarder, conserver, transmettre au public par télécommunication ou par l'Internet, prêter, distribuer et vendre des thèses partout dans le monde, à des fins commerciales ou autres, sur support microforme, papier, électronique et/ou autres formats.

L'auteur conserve la propriété du droit d'auteur et des droits moraux qui protège cette thèse. $\mathrm{Ni}$ la thèse ni des extraits substantiels de celle-ci ne doivent être imprimés ou autrement reproduits sans son autorisation.
In compliance with the Canadian Privacy Act some supporting forms may have been removed from this thesis.

While these forms may be included in the document page count, their removal does not represent any loss of content from the thesis.
Conformément à la loi canadienne sur la protection de la vie privée, quelques formulaires secondaires ont été enlevés de cette thèse.

Bien que ces formulaires aient inclus dans la pagination, il n'y aura aucun contenu manquant.

\section{Canadä}




\begin{abstract}
The architectural design of plants is selected by nature for optimal survival and reproductive fitness. A major goal of plant developmental biologists is to identify genes that control plant shape and form and to understand how these genes interact to create structural diversity. BLADE-ON-PETIOLE1 (BOP1) and BOP2 are an ancient and conserved set of plant-specific transcriptional co-regulators that play a key role in determining the architecture of leaves, inflorescences, and fruits in the model plant species, Arabidopsis thaliana. Phenotypes in bop1 bop 2 loss-of-function mutants include leafy petioles, asymmetric flowers subtended by a bract, lack of floral-organ abscission, and the partial conversion of flowers to shoots. Conversely, BOP1/2 gain-of-function mutants have severe defects in internode elongation resulting in short, compacted inflorescences. However, the mechanisms behind these architectural variations have yet to be determined. In my thesis, I show that $B O P 1 / 2$ are expressed in initiating lateral organ primordia during both the vegetative and reproductive phases of Arabidopsis development. As these primordia separate from the meristem, $B O P$ expression stably shifts to the proximal boundary region that separates these primordia from the meristem or from adjacent structures. In floral meristems, I show that BOP1 and BOP2 function redundantly with LEAFY, a master regulator of floral-meristem identity, to promote the expression of APETALAl, a key marker of commitment to floral fate. All three activities then converge in the floral meristem during stages 2-3 to down-regulate the expression of inflorescence identity genes including AGAMOUS-LIKE24 to promote the formation of a determinate floral shoot. In leaves, I show that BOP1 and BOP2 in the petiole function redundantly with leaf patterning factors ASYMMETRIC LEAVES1 (AS1) and AS2 to
\end{abstract}


maintain the stable repression of Class I KNOTTED1-LIKE HOMEOBOX (KNOXI) meristematic genes to facilitate simple leaf formation. In inflorescences, I show that the expression of BOP genes and the KNOXI family member KNOTTED-LIKE FROM ARABIDOPSIS THALIANA6 (KNAT6) are confined to pedicel axils (a boundary that separates the pedicel from the stem) by the combined activities of the KNOXI family member BREVIPEDILLUS (BP) and its interacting partner PENNYWISE (PNY). My data show that $B O P 2$ and $K N A T 6$ expression domains are differentially enlarged in $b p$ and $p n y$ mutants, corresponding to the distinctive patterns of short internodes, clustered or downward-oriented siliques, and defects in epidermal cell differentiation characteristic of these mutants. These data indicate that BP-PNY and BOP1/2-KNAT6 have reciprocal functions in the inflorescence. Further evidence shows that BOP1/2 are positive regulators of KNAT6 expression and that this regulation may be direct. Collectively, my work shows that BOP1/2 are key regulators of determinacy in lateral shoot and organ primordia and illustrate that changes in determinacy lead to dramatic architectural variation in these structures. My data also indicate that BOP1/2-KNAT6 (boundary factors) and BP-PNY (meristematic factors) have antagonistic functions in the inflorescence stem: the combined activities of BP-PNY restrict BOP1/2-KNAT6 to pedicel axils to facilitate the production of an inflorescence in which lateral branches and flowers are equally spaced along elongated internodes. Future studies will determine if differences in BOP1/2 activity or expression pattern form a common module for creating architectural diversity in plant species. 


\section{Acknowledgements}

It is a pleasure to thank all the people who made this thesis possible.

I am heartily thankful to my supervisor, Dr. Shelley Hepworth for her encouragement, guidance, and support during my research and study at Carleton University. In the five years since arriving from China to begin my $\mathrm{Ph} . \mathrm{D}$., she has taught me from the beginning about Arabidopsis as a model system and encouraged me to read widely, to improve my technical skills, and to try new techniques. Not only this, she has been patiently training me in critical thinking and academic writing. She was always accessible and willing to help me not only with my research but also with my family. Her perpetual energy and enthusiasm for research has motivated me.

I am also grateful to Dr. Owen Rowland and Dr. Anne-Gaëlle Rolland-Lagan for serving on my committee and providing helpful suggestions. Furthermore, I would like to thank the members of Dr. Hepworth's lab and Dr. Rowland's lab for collaborating with me over the years, and friends from other labs on my way. Special thanks to Jhadeswar Murmu, Paul Tabb, Madiha Khan, Sollapura Vishwanath (Vishwa), Ian Pulsifer, and Ashley Fournier, for thoughtful discussions on lab techniques.

Finally, I would like to acknowledge the support of my mom, dad, sister, husband, and daughter. I owe my deepest gratitude to my husband Tieqiang for his love, support, help and encouragement, and my daughter Ziyi, for her gentleness and understanding that mom has to come home late and work on weekends sometimes. 


\section{Preface}

The purpose of this thesis is to dissect the role of BLADE-ON-PETIOLE1 and 2 in patterning the Arabidopsis thaliana leaf and inflorescence at molecular level. I have carried out most of the experiments to elucidate how BOP1/2 interact with other growth regulators to sculpt the Arabidopsis thaliana leaf and inflorescence. However, some parts were carried out in collaboration with other graduate students or technicians; some Figures in Chapterl are adapted from published sources with permissions from the publishers for better understanding of the knowledge. Detailed information concerning these contributions to my thesis are discussed below.

\section{Papers published or submitted at the time of thesis submission}

1. Xu, M., Hu, T., McKim, S., Murmu, J., Haughn, G.W., and Hepworth, S.R. (2010) Arabidopsis BLADE-ON-PETIOLE1 and 2 promote floral meristem fate and determinacy in a previously undefined pathway targeting APETALA1 and AGAMOUSLIKE24. Plant Journal 63, 974-989. This paper was published in July, 2010 and was reprinted in my thesis as Chapter 2 with permission of the publisher (John Wiley and Sons).

2. Khan, M., Xu, M., Hu, T., Tabb, Murmu, J., McKim, S.M., Story, K., Mercado, J., and Hepworth, S.R. Antagonistic interaction of BLADE-ON-PETIOLE1 and 2 with BREVIPEDICELLUS and PENNYWISE regulates Arabidopsis inflorescence architecture. This manuscript was submitted to the Plant Cell on October $30^{\text {th }}, 2010$ and the submission number is: PLANTCELL/2010/080747. I am a co-first author of this 
manuscript and we are revising it according to the reviewers' comments. In my thesis I only presented the work that I did or work done by technicians or students that I cosupervised. The manuscript is about the role of BOP1/2 in Arabidopsis leaf and inflorescence patterning. However, I divide it into two chapters in my thesis: Chapter 3 is about the role of BOP $1 / 2$ in leaf patterning and Chapter 4 is about the role of BOP $1 / 2$ in inflorescence patterning. Detailed information about the contributions to this thesis is presented below.

\section{Statement of contributions}

I have contributed to the research described in this thesis as followers:

1. In Chapter 2, Dr. Hepworth and I formulated the hypothesis and I carried out all of the experiments described in this Chapter with the following exceptions: Sarah McKim provided the scanning electron micrographs and BiFC data, Tieqiang $\mathrm{Hu}$ provided the qRT-PCR data for $A P 1, A G L 24, S O C 1$, and FUL transcripts, and Jhadeswar Murmu made the pBOP1::BOP1-GFP plants used for ChIP assays. Dr. Hepworth and I analyzed the data and prepared the manuscript.

2. In Chapter3, Dr. Hepworth and I formulated the hypothesis. I co-supervised Tieqiang $\mathrm{Hu}$ (Technician) and Jethro Mercado (undergraduate) with Dr. Hepworth for the following experiments: Jethro Mercado helped with the isolation of bopl bop2 as 1 and bopl bop2 as 2 triple mutants and I analyzed the plants and collect the data; Tieqiang Hu performed the qPCR experiment on $S T M, B P, K N A T 2, K N A T 6$ and $P N Y$ and Dr. Hepworth analyzed the data; Madiha Khan made the bop1 bop2 
KNAT2::GUS and bop1 bop2 KNAT6::GUS lines and I used them for GUS staining and sectioning.

3. In Chapter4, Dr. Hepworth and I formulated the hypothesis and I carried out all of the experiments described in this Chapter except that Madiha Khan isolated the BOP2::GUS bp-2 and BOP2::GUS pny lines and I used them for whole mount GUS staining and sectioning. I initiated the project for this chapter with the crosses bopl bop $2 \times$ bp-1; and bopl bop $2 \times$ pny. I co-supervised Madiha Khan with Dr. Hepworth to isolate bop1 bop 2 bp and bopl bop 2 pny triple mutants. She found that the defects in $b p$ and pny were rescued by bopl bop2. We collaborated on this project and I did the qPCR of $K N A T 6$ and $B P$ in various tissues and I did the expression analysis of $B O P, K N A T 6, B P$ and $C U C 3$ in different genotypes.

4. With the help of Dr. Hepworth, I prepared the manuscript of this thesis.

\section{The acknowledgement of the use of Copyright material}

As the first author of Xu et al. Plant Journal (2010), I reprinted it and enclosed it as Chapter 2 in this thesis with the permission from the publisher John Wiley and Sons. I also adopted some figures from published papers in Chapter 1 with permission from the publishers. I redrew Figure 1.3 from Barton (2010) with permission from the publisher Elsevier; I reprinted Figure 1.4 from Rast and Simon (2008) with permission from the publisher Elsevier; I reprinted Figure 1.5 from Byrne (2006) with permission from the publisher PLoS Genetics. 


\section{List of Abbreviations}

\begin{tabular}{|c|c|}
\hline $\mathrm{ABRC}$ & Arabidopsis Biological Resource Center \\
\hline $\mathrm{AG}$ & AGAMOUS \\
\hline AGL24 & AGAMOUS-LIKE24 \\
\hline ANK & Ankyrin \\
\hline AP1 & APETALA1 \\
\hline AP2 & APETALA2 \\
\hline AP3 & APETALA3 \\
\hline AS1 & ASYMMETRIC LEAVES1 \\
\hline AXR1 & AUXIN RESISTANT1 \\
\hline ATH1 & ARABIDOPSIS THALIANA HOMEOBOX1 \\
\hline BEL1 & BELL1 \\
\hline $\mathrm{BiFC}$ & Biomolecular Fluorescence Complementation \\
\hline BOP1 & BLADE-ON-PETIOLE1 \\
\hline $\mathrm{BP}$ & BREVIPEDICELLUS \\
\hline $\mathrm{BTB} / \mathrm{POZ}$ & $\begin{array}{l}\text { Broad-Complex, Tramtrack, and Bric-a-Brac/POX virus and Zinc } \\
\text { finger }\end{array}$ \\
\hline $\mathrm{bZIP}$ & basic leucine zipper \\
\hline $\mathrm{CAL}$ & CAULIFLOWER \\
\hline $\mathrm{CHX}$ & Cycloheximide \\
\hline ChIP & Chromatin Immunoprecipitation \\
\hline CK & Cytokinin \\
\hline $\mathrm{CL}$ & Continuous Light \\
\hline
\end{tabular}




\begin{tabular}{ll} 
CLV & CLAVATA \\
CO & CONSTANS \\
Col & Columbia \\
CUC & CUP-SHAPED COTYLEDONS \\
Cys & Cysteine \\
CZ & Central Zone \\
EREBP & Ethylene response element binding protein \\
EYFP & Enhanced Yellow Florescent Protein \\
FD & FLOWERING LOCUS D \\
FIL & FILAMENTOUS FLOWER \\
FLC & FLOWERING LOCUS C \\
FMI & Floral Meristem Identity \\
FOI & Floral Organ Identity \\
FT & FLOWERING LOCUS T \\
FUL & Inflorescence Meristem \\
GFP & FRUITFULL \\
GA & Green Florescent Protein \\
GAPC & Gibberellic Acid \\
GUS & Glyceraldehyde-3-phosphate dehydrogenase \\
HM-ZIP & homeodomain leucine-zipper \\
\hline &
\end{tabular}




\begin{tabular}{|c|c|}
\hline $\mathrm{PM}$ & Primary Morphogenesis \\
\hline SM & Secondary Morphogenesis \\
\hline JAG & JAGGED \\
\hline KAN & KANADI \\
\hline KNAT & KNOTTED-LIKE FROM ARABIDOPSIS THALIANA \\
\hline KNOX & KNOTTED-LIKE HOMEOBOX \\
\hline KNOXI & Class I KNOTTED-LIKE HOMEOBOX \\
\hline Ler & Landsberg erecta \\
\hline LFY & LEAFY \\
\hline LOB & LATERAL ORGAN BOUNDARIES \\
\hline LBD & LATERAL ORGAN BOUNDARIES DOMAIN \\
\hline LOF & LATERAL ORGAN FUSION \\
\hline $\mathrm{LD}$ & Long Day \\
\hline LMI & LATE MERISTEM IDENTITY1 \\
\hline \multirow[t]{2}{*}{ MADS-box } & MCM1-AGAMOUS-DEFICIENS-SERUM RESPONSE \\
\hline & FACTOR conserved sequence motif \\
\hline NPA & N-napthylphthalamic acid \\
\hline NPR1 & NON-EXPRESSOR OF PATHOGENESIS RELATED GENES1 \\
\hline $\mathrm{OC}$ & Organizing Centre \\
\hline PAN & PERIANTHIA \\
\hline PCR & polymerase chain reaction \\
\hline PHB & PHABULOSA \\
\hline PHV & PHAVOLUTA \\
\hline
\end{tabular}


PI

PIN1

PNF

PNY

PR1

PTL

PZ

qPCR

RT-PCR

$\mathrm{RZ}$

SA

SAM

SAR

SD

SEM

s.e.m.

SEP

SOC1

STM

STP

SPL

SVP

TGA
PISTILATTA

PIN-FORMED1

POUNDFOOLISH

PENNYWISE

PATHOGENESIS-RELATED1

PETAL LOSS

Peripheral Zone

Quantitative RT-PCR

Reverse transcriptase polymerase chain reaction

Rib Zone

Salicylic Acid

Shoot Apical Meristem

Systemic Acquired Resistance

Short Day

Scanning Electron Microscopy

standard error of mean

SEPALLATA

SUPPRESSOR OF OVEREXPRESSION OF CONSTANS1

SHOOT MERISTEMLESS

STAMINA PISTILLOIDA

SQUAMOSA PROMOTER BINDING PROTEIN-LIKE

SHORT VEGETATIVE PHASE

TGACG-BOX BINDING 


$\begin{array}{ll}\text { TALE } & \text { Three-Amino Acid-Loop-Extension } \\ \text { UFO } & \text { UNUSUAL FLORAL ORGANS } \\ \text { UNI } & \text { UNIFOLIATA } \\ \text { UTR } & \text { Untranslated region } \\ \text { YAB } & \text { YABBY } \\ \text { Y2H } & \text { Yeast-Two-Hybrid } \\ \text { WT } & \text { Wild-Type } \\ \text { WUS } & \text { WUSCHEL }\end{array}$


Genetic Nomenclature in Arabidopsis thaliana

$B O P 1 \quad$ wild-type gene

BOP1 gene product or activity

bopl mutant gene allele (loss-of-function)

bop1-6D mutant gene allele (gain-of-function) 
Chapter 1

General introduction 


\subsection{Overview of thesis}

Plants of a species show a characteristic patterning for leaves, inflorescences, and flowers that is selected for in nature to optimize reproductive success. Forward and reverse genetics approaches in the model plant species, Arabidopsis thaliana, have identified many genes that control plant architecture. However, it remains to be seen how many of these genes function at the molecular level and how their activities interact to generate structural diversity.

The aerial parts of a plant are generated through the activity of the shoot apical meristem (SAM), a self-renewing population of stem cells located at the growing shoot tip. During Arabidopsis vegetative development, the SAM generates leaves without internode elongation to produce a compact rosette. Upon floral induction, internode elongation is allowed and the SAM (now known as the inflorescence meristem, IM) transitions to produce lateral branches and then flowers (reviewed in Bowman and Eshed, 2000; Barton, 2010). However, the patterning of these organs and shoots varies between plant species.

The main objective of this thesis is to investigate the role of two conserved plant-specific transcriptional co-regulators, BLADE-ON-PETIOLE1 (BOP1) and BOP2, which control the architecture of leaves, inflorescences, and flowers in plants. $B O P 1$ and $B O P 2$ (referred to as $B O P 1 / 2)$ are expressed in emerging leaf and floral primordia. As these primordia partition from the SAM, BOP $1 / 2$ expression shifts to the boundary at the base of the organ where expression is maintained thereafter. This pattern of expression suggests a role for these genes in organ initiation and boundary patterning. Loss-of-function bopl bop 2 mutations cause a range of patterning defects including leafy petioles, asymmetric flowers subtended by a bract, and the partial conversion of flowers to shoots. Conversely, gain-of-function BOP1/2 plants are 
restricted in stem growth and have compact internodes. While it is clear that these genes control plant architecture, how they function at the molecular level is largely unknown.

To address this, I used a combination of molecular, genetic and biochemical approaches to elucidate how BOP1/2 interact with developmental-specific regulators to influence (a) floral meristem identity, (b) leaf patterning, and (c) internode elongation. As a background to understand the rationale and significance of these studies, I will first provide an overview of Arabidopsis development with an emphasis on plant architecture. I will then explain how BOP1/2 interact with other regional and developmental-specific factors to promote floralmeristem identity, simple leaf shape, and elongation of internodes in inflorescences.

\subsection{Study species}

In the late 1970's, Arabidopsis thaliana was selected as a model species to study plant development for four main reasons:

a) It is a diploid organism with a small compact genome compared to rice or wheat.

b) It is easy to obtain Arabidopsis mutants and to make transgenic plants.

c) It is easy to grow in a lab because its size is small $(30-40 \mathrm{~cm})$, its life-time is short $(6-8$ weeks), and it has high seed set.

d) It can be self- or cross-fertilized at will (Meyerowitz, 1987).

Since then, the Arabidopsis genome has been completely sequenced and key resources such as a library of T-DNA insertion mutants are readily available from the Arabidopsis Biological Resource Center (ABRC). Although Arabidopsis has no economic value, it is in the same family as cabbage, broccoli, and horseradish. Information discovered about this plant can be applied to modify plants or accelerate breeding for human benefit (Jack, 2004). 


\subsection{The life cycle of Arabidopsis thaliana}

The life cycle of the flowering plant Arabidopsis can be broadly divided into three developmental phases: an embryogenesis phase, a vegetative phase and a reproductive phase. After germination, Arabidopsis undergoes vegetative development and a compact rosette is formed from the activities of the meristem established from embryogenesis (Fletcher, 2002; Figure 1a). Upon perceiving endogenous and environmental cues, the plants stops making leaves and transits to generate flowers, initiating the reproductive phase of development. Reproductive development itself has two distinct stages (Ratcliffe et al., 1998): the first-inflorescence (FI) stage and the second-inflorescence (SI) stage. During the FI stage, several cauline leaves are produced, each in association with a secondary inflorescence (Ratcliffe et al., 1998; Figure 1b). During the SI stage, leaf development is fully repressed and flowers are produced without leaves (Figure 1b). Some biologists consider the FI stage as part of the vegetative phase, since cauline leaves are still produced (Liljegren et al., 1999). In this study, however, we followed the convention of Ratcliffe et al. (1998) who defined the period of cauline leaf growth as part of the reproductive phase because mutations in some genes specifically affect the transition from indeterminate secondary inflorescences to determinate flowers.

As explained above, the above-ground parts of a plant are generated post-embryonically by the apical meristem. Each set of structures produced by this meristem can be viewed as a standard module comprised of three parts: a leaf, an axillary meristem, and an internode. How the parts of each module are elaborated however, depends on the plant species and the phase of development (Sussex, 1989). For example, during the vegetative phase of Arabidopsis development, a leaf with the potential to form an axillary meristem is developed but internode elongation is repressed. During the first-inflorescence stage of reproductive development, the 
module is fully developed and consists of a cauline leaf, an axillary meristem that develops into a secondary inflorescence, and an elongated internode. During the second-inflorescence stage of reproductive development, leaf development is fully suppressed, while the axillary meristem develops as a flower, and the internode is elongated. The position of each module and its subsequent development is strongly influenced by the environment and by genetic cues, allowing the overall architecture of a plant to unfold over developmental time (Sussex, 1989).

\subsection{Establishment and maintenance of the shoot apical meristem}

Unlike animals, where the body plan is completed during embryogenesis, organs and tissues are continuously produced over the life cycle of a plant. New organs or tissues are initiated from groups of stem cells located at the shoot tip or root tip of a plant (Fletcher, 2002). As explained earlier, the above-ground parts of the plant are built from a series of repeating modules that are produced one-at-a-time by the SAM. This strategy works well for plants because they are immobile and must continually respond to the changing environment to thrive. Their modular make-up gives them the flexibility to adapt by changing their patterns of growth and development (Fletcher, 2002).

\subsubsection{Organization of the shoot apical meristem}

Two apical meristems, the shoot apical meristem (SAM) and the root apical meristem (RAM), are established during embryogenesis and determine the architecture of aerial and underground parts of a plant, respectively. The RAM will not be considered further. The SAM is organized into three distinct zones on the basis of morphological differences and cell division rates: the central zone (CZ), the peripheral zone (PZ), and the rib zone (RZ) (Figure 1.2). The CZ cells are large and highly vacuolated, and they lie at the very centre of the SAM. These cells divide 
infrequently relative to other cells in the SAM. Surrounding the CZ is the PZ. The PZ cells are smaller and divide more frequently than the $\mathrm{CZ}$ cells. Importantly, the $\mathrm{PZ}$ cells give rise to lateral organs that build up the aerial body of a plant; thus this zone represents a transitional region of the SAM. Subtending the $\mathrm{CZ}$ is the $\mathrm{RZ}$ and its cells are large and vacuolated. Cell division in this zone is suppressed during vegetative development and activated during reproductive development giving rise to internodes. Plants can maintain a reservoir of stem cells and continuously produce lateral organs on the flanks of the meristem by coordinating cell fate at different zones: the progeny of $\mathrm{CZ}$ cells either stay in the centre of the SAM and maintain their stem cell activity, or they enter the PZ or RZ and provide founder cells for the formation of lateral organs or internodes (reviewed in Liljegren and Yanofsky, 1996; Bowman and Eshed, 2000; Fletcher, 2002; Barton, 2010).

The SAM can also be subdivided into distinct L1, L2 and L3 cell layers (Figure 1.2). The outmost cell layer in Arabidopsis is termed the L1 layer. The L2 cell layer is the sub-epidermal layer of cells underlying the L1 layer. Underneath the L2 layer is the L3 layer, which is comprised of multiple cell layers. The L1 and L2 layers constitute the tunica of the SAM, whereas the L3 layers constitute the corpus of the SAM. Cells from the L1, L2, and L3 layers encompass the CZ and PZ, while the RZ consists of cells from the L3 layer (Fletcher, 2002; William and Fletcher, 2005; Barton, 2010).

\subsubsection{Establishment and maintenance of the shoot apical meristem}

Because of the significance and complexity of the SAM in plant development, biologists are very interested in knowing how the SAM is established and maintained throughout the plant life cycle. Two transcriptional regulators, WUSCHEL (WUS) and SHOOT MERISTEMLESS (STM) from 
the Class 1 KNOTTED1-LIKE HOMEOBOX (KNOX1) family are key factors in the establishment and maintenance of the SAM.

\subsubsection{WUSCHEL functions through CLAVATA signaling in the establishment and maintenance of the SAM}

Molecular and genetic data support the idea that WUS is a master regulator for maintenance of the SAM. WUS encodes a homeodomain (a highly conserved protein motif that binds to DNA and regulates gene expression subsequently) transcription factor, and loss of WUS function results in failure to maintain the $\mathrm{CZ}$, indicating a central role for WUS in maintaining the reservoir of stem cells (Mayer et al., 1998). WUS is not expressed in CZ, but rather in a small group of cells underneath termed the Organizing Centre (OC) (Laux et al., 1996).

Maintenance of the stem cell population relies on a WUS-mediated inductive signal, which must be targeted to the overlying apical cells. Members of the CLAVATA (CLV1, CLV2, and CLV3) family of signaling proteins interact to maintain the size and position of the stem cell population. $C L V 1$ and $C L V 2$ encode receptor-like proteins that are expressed primarily in the L3 corpus layers. The CLV3 gene encodes a small, diffusible secreted ligand that expresses in the L1 and L2 tunica cells of the SAM, corresponding to the CZ (Clark et al., 1993; 1996; Kayes et al., 1998). CLV3 interacts with the CLV1-CLV2 receptor complex in the underlying L3 layer cells. The CLV proteins negatively regulate WUS expression, thereby limiting the size and position of the OC (Williams and Fletcher, 2005).

There is antagonistic activity between WUS and CLV regulators in the establishment and maintenance of stem cells (Fletcher, 2002; William and Fletcher, 2005). Loss-of-function in CLV1, CLV2, or CLV3 genes causes enlarged shoot and floral meristems throughout development, opposite to loss-of-function in WUS. In addition, WUS expression is not restricted 
to the OC and expands laterally in clv3 mutants, while there is no WUS expression in CaMV35S::CLV3 transgenic plants (Fletcher et al., 1999; Brand et al., 2000; Schoof et al., 2000), indicating that CLV3 represses WUS.

Taken together, a WUS-CLV feed-back loop is present in the SAM (Figure 1.3): WUS activity in the $\mathrm{OC}$ specifies the overlying cells as stem cells thereby inducing $C L V 3$ expression. Consequently, activation of the CLV signaling complex feeds back to limit the domain of WUS expression. Any change in CLV3 or WUS expression levels cause subsequent adjustments in other gene expression levels and in SAM activity.

\subsubsection{Class I KNOX proteins function with BEL1-like homeodomain proteins in the establishment and maintenance of the SAM}

Class $1 K N O X$ genes ( $K N O X 1$ genes) are another class of positive regulators of SAM formation and maintenance. KNOXI genes encode the homeodomain-containing proteins SHOOT MERISTEMLESS (STM), BREVIPEDICELLUS (BP) (also known as KNOTTED-LIKE FROM ARABIDOPSIS THALIANA1, KNAT1), KNAT2 and KNAT6. STM is expressed throughout the SAM and has a central role in meristem maintenance since strong alleles of stm are unable to establish and maintain a functional SAM (Long et al., 1996). BP is expressed in the PZ of the SAM and at the boundaries of leaf primordia (Lincoln et al., 1994; Ori et al., 2000) while KNAT6 is expressed in the embryonic SAM and in the adaxial part of the boundary that separates leaf primordia from the SAM (Belles-Boix et al., 2006). Neither $b p$ nor knat6 mutants display any SAM defects, but their mutations enhance the phenotype of plants with weak stm alleles to give a strong stm mutant phenotype (Byrne et al., 2002; Belles-Boix et al., 2006). These findings reveal that BP and KNAT6 have roles in maintaining the SAM in addition to STM. A third gene, KNAT2, is expressed at the base of the SAM but its role in the SAM is 
unclear since its mutation does not enhance the phenotype of weak stm mutants (Byrne et al., 2002; Belles-Boix et al., 2006).

Gain-of-function studies further support the idea that $K N O X 1$ genes promote meristem formation. Misexpression of any KNOX1 family member induces ectopic meristem formation (no organogenesis) on the adaxial surface or margins of leaves (Lincoln et al., 1994; Chuck et al., 1996, Pautot et al., 2001; Gallois et al., 2002; Dean et al., 2004). However, combined misexpression STM and WUS induces ectopic organogenesis on the hypocotyl and cotyledons, demonstrating that the KNOX1 and WUS-CLV systems have complementary roles in meristem formation (Gallois et al., 2002; Barton, 2010).

Biochemical studies show that KNOX1 proteins interact with members of the related BELL1-like class of homeodomain proteins to perform their function in the SAM (Belloui et al., 2001; Byrne et al., 2003; Smith and Hake 2003; Kanrar et al., 2006; Rutgens et al. 2009). Three BELL1-like members are expressed in the SAM: PENNYWISE (PNY), POUNDFOOLISH (PNF) and ARABIDOPSIS THALIANA HOMEOBOX1 (ATH1) (Kanrar et al., 2006; Rutjens et al., 2009). Loss-of function pny, but not pnf, enhances the phenotype of plants with a weak stm allele and when all three loci are mutated, the meristem is not maintained (Byrne et al., 2003; Smith and Hake 2003; Kanrar et al., 2006; Rutgens et al. 2009). These data indicate that SAM establishment and maintenance requires the activity of KNOX1-BELL heterodimers.

A few of the KNOX1 transcriptional targets have been uncovered recently, revealing how KNOX1 proteins function for the establishment and maintenance of the SAM. KNOX1 proteins directly activate cytokinin (CK) biosynthesis genes or gibberellin (GA) deactivation genes to modulate the abundance of the plant hormones CK and GA. Activation of STM can rapidly increase the transcription level of ISOPENTENYL TRANSFEREASE7 (IPT7), a CK biosynthesis 
gene (Yanai et al., 2005). As a result, CK accumulation levels are elevated (Yanai et al., 2005).

Similarly, GA deactivation genes $A t G A 2 o \times 2$ and $A t G A 2 o x 4$ are activated in response to STM induction, resulting in decreased GA accumulation (Jasinski et al., 2005). Therefore, a high CK to GA ratio in the SAM is yielded by activation of CK biosynthesis and GA deactivation genes. Elevating CK biosynthesis can partially rescue stm mutant phenotypes, and reducing CK levels or elevating GA levels can enhance stm mutant phenotypes, again suggesting that STM exerts its effect by regulating CK and GA abundance (Jasinski et al., 2005; Yanai et al., 2005). Interestingly, plants overexpressing CK display high levels of $S T M$ and $B P$ transcripts and phenocopy 35S::STM or 35S::BP plants (Chuck et al., 1996; Rupp et al., 1999; Gallois et al. 2002) suggesting that $C K$ acts in a feed-forward loop targeting $S T M$ and $B P$ for establishment and maintenance of the SAM.

In summary, the SAM is central to plant development because it gives rise to all aerial parts of the plant. WUS and KNOX1 proteins are the major regulators of SAM establishment and maintenance and they work co-operatively to perform this task. WUS function is through a WUS-CLV feed-back loop, whereas KNOX1 proteins form heterodimers with BELL homeodomain proteins and function in part by elevating CK levels and lowering GA levels.

\subsection{Boundary establishment}

When lateral organ primordia are partitioned from the SAM, a boundary develops in the form a narrow groove that separates the primordia from the meristem (Figure 1.4, Aida and Tasaka 2006ab; Rast and Simon, 2008). Boundaries are first established between the embryonic SAM and the cotyledons, and the pattern is repeated for all subsequent lateral organs as they emerge from the SAM. Cells in the boundary zone divide at a slower rate than cells in adjacent domains indicating that growth is restricted in this zone (Breuil-Broyer et al., 2004; Aida and Tasaka, 
2006ab). Members of the CUP-SHAPED COTYLEDON (CUC), LATERAL ORGAN BOUNDARIES DOMAIN (LBD) (defined by an expression domain at lateral organ boundaries), LATERAL ORGAN FUSION (LOF), BOP, and KNOXI gene families (e.g. KNAT6) all display boundary-localized expression patterns and their combined activities are required for: a) organ separation, b) meristem maintenance, and c) boundary cell specialization (reviewed in Aida and Tasaka, 2006ab; Rast and Simon, 2008). Boundaries often contain specialized cell types that are important later in plant development. For example, cells in the axils of leaves have the potential to form axillary meristems (McSteen and Leyser, 2005) whereas cells at the base of floral organs are specialized for abscission (Lewis et al., 2006; McKim et al., 2008). Boundary genes are therefore important regulators of plant architecture.

The NAC (for NAM, ATAF1, and CUC2) domain transcription factors encoded by $C U C 1, C U C 2$, and $C U C 3$ restrict growth and are viewed as the central regulators of boundarycell identity. Genetic data show that the $C U C$ genes are redundantly required for organ separation and meristem initiation (Aida et al., 1997; Vroemen et al., 2003; Aida and Tasaka, 2006ab; Hibara et al., 2006). $C U C 1$ and $C U C 2$ are activated early in embryogenesis and promote meristem initiation by activating STM. Once activated, $S T M$ feeds back to restrict $C U C 2$ to the boundary, thus separating meristem and organ domains in the embryo (Aida et al., 1997; 1999; Takada et al., 2001). Accumulating auxin in the developing cotyledon tips additionally restricts CUC2 expression to the boundary (Aida et al., 2002; Furutani et al., 2004). Inactivation of CUC genes in Arabidopsis results in fused cotyledons (cup-shaped cotyledons) and a blunted stem apex lacking a SAM (Aida et al., 1999; Hibara et al., 2006). CUC genes are expressed earlier than KNAT6 and correct KNAT6 expression requires CUC activity (Belles-Boix et al., 2006) suggesting that $C U C$ genes play a role in establishing the unique transcriptional profile of the 
boundary, but direct transcriptional targets are still unknown. KNAT6 contributes to boundary maintenance, as evidenced by severe defects in cotyledon separation in the stm-2 knat6 double mutant (Belles-Boix et al., 2006).

During reproductive development, the CUC genes promote the formation of axillary meristems since some $c u c$ mutants sometimes lack secondary inflorescences in the axil of cauline leaves (Hibara et al., 2006). LOF1 and LOF2 encode MYB transcription factors (for Myeloblast, an oncogene first identified in avian myloblastosis virus) that are additionally required for separation of the cauline leaf and secondary inflorescence: these structures are fused together in the double mutant (Lee et al., 2009). Combined loss-of-function lof and cuc mutants display enhanced organ fusion defects relative to single mutants and are more often unable to form secondary shoots in the axils of cauline leaves (Lee et al., 2009). Organ fusions are also frequent in the bopl bop 2 mutant during reproductive development (data not shown) and the cellular structure of pedicel-stem junction is disrupted (McKim et al., 2008) indicating that BOP1/2 contribute to organ separation and boundary patterning in reproductive development. However, defects in organ separation and meristem maintenance are not seen in bop1 bop2 mutant seedlings suggesting that $\mathrm{BOP} 1 / 2$ activity is more important during reproductive development.

A common theme among genes expressed in the boundary is their modulation of $K N O X I$ expression. As explained above, $C U C 1$ and $C U C 2$ genes promote $S T M$ expression in the apex of globular stage embryos to establish the embryonic meristem (Aida et al., 1997; 1999; Tasaka et al., 2001). In addition, misexpression of $C U C 1$ and the LBD family member JAGGED LATERAL ORGANS (JLO) direct the ectopic expression of BP when misexpressed in leaves (Takada et al., 2001; Borghi et al., 2007) and CUC and LOF activities are required to reactivate 
STM expression for the formation of axillary meristems in cauline leaf axils (Lee et al., 2009). These data define $C U C, L O F$, and $J L O$ as positive regulators of meristem formation. Conversely, BOP1/2 and ASYMMETRIC LEAVES2 (AS2; another LBD family member) negatively regulate KNOX1 expression in leaves (Ori et al., 2000; Ha et al., 2003; 2007; 2010; Jun et al. 2010;) defining these boundary genes as negative regulators of meristem activity. These data suggest that interplay between boundary genes maintains an appropriate balance between meristem activity and cell differentiation. This balance is important as meristem activity and organ differentiation are two competing processes that must be compartmentalized in the shoot apex for the continuous production of modules by the SAM.

In summary, boundary cells have a unique transcriptional profile and provide a physical barrier between meristematic and non-meristematic cells in the plant. The combined activities of CUC, LOF, LBD, BOP1/2, and KNAT6 proteins are required to restrict growth, regulate meristematic potential, achieve organ separation, and promote the differentiation of boundary cells for functions such as axillary meristem formation or abscission. It remains unclear how genes in the boundary coordinate the competing processes of meristem maintenance and organ production.

\subsection{Leaf initiation and architecture}

Leaf development can be divided into the three stages termed leaf initiation (I), primary morphogenesis (PM), and secondary morphogenesis (SM). During stage I, organ phyllotaxy and growth rate are established, and during the PM and SM stages, the architecture of the leaves is established (Hay and Tsiantis, 2010). Leaf primordia may also be described according to morphological stages in their development, defined as P0, P1, P2, P3, and P4 (Long and Barton, 2000). These stages are defined as follows: P0, when an STM-negative region first appears on 
the flanks of the SAM; P1, when a lateral bulge is seen on the flanks of the SAM; P2, when a visible cleft is seen between the primordia and the SAM; P3, when primordia extend farther apically and a deep cleft separates the primordia and the SAM; and P4, when the primordia has extended and curves over to about the midpoint of the SAM. A summary of the regulators for leaf initiation and leaf patterning (leaf shape and form) is provided next.

\subsubsection{Leaf initiation}

In Arabidopsis, leaf primordia are initiated on the flanks of the SAM in a spiral phyllotactic pattern (Reinhardt et al. 2000; Byrne et al., 2003). Organs are initiated at sites of high auxin response in the PZ called auxin maxima (Reinhardt et al., 2000; 2003; Benkova et al., 2003). The orientation of PIN-FORMED (PIN) auxin efflux facilitators, particularly PIN1, play a crucial role in draining auxin from the SAM epidermis and transporting it towards maxima that predict the site of new organ initiation (Gälweiler et al., 1998; Benková et al., 2003; reviewed in Vernoux et al., 2010). To initiate a new organ, KNOXI meristematic and $C U C$ boundary genes are switched off in an auxin-dependent manner (Vernoux et al., 2000; Furutani et al., 2004; Aida et al., 2002; Vernoux et al., 2010). At the same time, genes that promote differentiation and maintain repression of $K N O X 1$ meristem genes, including $B O P 1 / 2, A S 1$, and $A S 2$ are switched on (Byrne et al., 2000; Ori et al., 2000; Semiarti et al., 2001; Iwakawa et al., 2002; Ha et al., 2003; 2007; Jun et al., 2010), together with genes that promote cell division, such as AINTEGUMENTA (ANT) (Long and Barton, 1998; 2000; reviewed in Rast and Simon, 2008).

Leaf initiation and stem-cell maintenance are two competing processes that are compartmentalized in the shoot apex. The SAM is marked by KNOXI gene expression (Lincoln et al., 1994; Long et al., 1996; Long and Barton, 2000). By contrast, $A S 1, A S 2$, and BOP1/2 are expressed in $\mathrm{P} 0$ and $\mathrm{P} 1$ leaf primordia where their activities maintain the repression $K N O X 1$ in 
the leaf primordia (Byrne et al., 2000; Ori et al., 2000; Semiarti et al., 2001; Iwakawa et al., 2002; Lin et al., 2003; Norberg et al., 2005; Ha et al., 2007; 2010; Jun et al., 2010).

ASl encodes a MYB transcription factor that is expressed on both abaxial and adaxial sides of the leaf primordia (Byrne et al., 2000) whereas AS2 is a member of the LBD family and is expressed on the adaxial side of the leaf primordia (Semiarti et al., 2001; Iwakawa et al., 2002; Jun et al. 2010). AS1 and AS2 bind to separate promoter sites in BP but interact in a complex that directs stable changes in the chromatin structure by recruiting the chromatin remodeling factor HIRA (Xu et al., 2003; Phelphs-Durr et al., 2005; Guo et al., 2008). BOPI and BOP2 encode BTB-ankyrin domain-containing transcriptional co-regulators that are expressed in a partly overlapping domain with $A S 1$ and $A S 2$ (Ha et al., 2004; Hepworth et al., 2005; Norberg et al., 2005; see also Chapter 3). Jun et al. (2010) showed that BOP1 directly promotes $A S 2$ expression to maintain $B P$ repression in leaves.

\subsubsection{Leaf patterning}

After stage I, leaf primordia proceed to the PM and SM stages for maturation. At these stages, leaf primordia are manipulated by various regulators that establish the adaxial-abaxial and proximal distal axes and determine leaf shape and form,

The adaxial-abaxial axis is established early in the development of leaf primordia. The dorsal or adaxial side of the leaf is the side that is next to the SAM whereas the ventral or abaxial side is the side faces away from the SAM (Figure 1.5a). Tricomes are a useful marker for identifying the adaxial side of the leaves since they are more abundant on the adaxial side (Byrne, 2006; Figure 1.5b). Adaxial identity is primarily specified by Class Ш HD-ZIP transcription factors, which share a common homeodomain DNA-binding motif (HD) and a leucine zipper dimerization motif (ZIP) (Figure 1.3). There are five class III HD-ZIP 
transcription factors in Arabidopsis, including PHABULOSA (PHB), PHAVOLUTA (PHV), ATHB8, ATHB15, and REVOLUTA (REV) (Byrne, 2006). The LBD transcription factor encoded by $A S 2$ and BTB-ankyrin transcriptional co-activator encoded by $B O P 1 / 2$, expressed at the adaxial base of leaf primordia (Norberg et al., 2005; Jun et al., 2010) also help specify adaxial leaf identity, mainly through regulating Class Ш HD-ZIP transcription factors (Xu et al., 2003; Ha et al., 2007). Abaxial identity is mainly specified by two families of transcription factors, the YABBY (YAB) family that includes FILAMENTOUS FLOWER (FIL) and the KANADI (KAN) family (Figure 1.3) (Kerstetter et al., 2001; Emery et al., 2003; Eshed et al., 2004).

In addition to adaxial-abaxial polarity, patterning along the proximal-distal axis further defines the architecture of leaves and primarily contributes to the diversity of leaf architectures. There are two major types of leaves, simple or compound, defined based on their degree of complexity along the proximal-distal axis. A leaf with several leaflets (each leaflet may or may not have a short petiolude) on a petiole is termed a compound leaf whereas a leaf with a single undivided flat blade (no leaflet) is termed a simple leaf. Accordingly, meristematic activity that causes growth in the petiole is allowed to different degrees in the compound leaves, whereas it is prohibited in the simple leaves (reviewed in Hasson et al., 2010; Uchida et al., 2010).

There are at least three interconnected pathways that control the simple-compound leaf deviation in plants. The first pathway involves the $K N O X 1$ genes. In simple leaf species such as Arabidopsis, STM and BP are expressed in the SAM but repressed in leaf primordia (Ori et al. 2000; Hay et al., 2006ab). However, in compound leaf species such as tomato and Cardamine, $S T M, K N A T 2$, and $B P$ are at first repressed but reactivated at later stages in the leaf primordia (Hay and Tsiantis, 2006b; Shani et al., 2010). Remarkably, inhibition of KNOX1 genes in tomato 
and Cardamine leaves results in less complicated compound leaves, whereas induction of KNOXI genes in Cardamine leaves results in more complicated compound leaves (Hay and Tsiantis, 2006b; Shani et al., 2010). In Arabidopsis, KNOXI genes are repressed by the overlapping activities of $\mathrm{AS} 1, \mathrm{AS} 2$, and $\mathrm{BOP} 1 / 2$ so that smooth petioles are formed (Byrne et al., 2000; Ori et al., 2000; Hay et al., 2006a; Ha et al., 2007; 2010). As stated above, AS1 and AS2 form a complex that recruits the chromatin-remodeling protein HIRA, leading to stable repression of BP and KNAT2 in developing leaves (Xu et al., 2003; Phelps-Durr et al., 2005; Guo et al., 2008). BOP1/2 is proposed to repress $K N O X 1$ genes indirectly based on the fact that BOP1 directly activates $A S 2$ and AS2 directly represses BP (Guo et al., 2008; Jun et al., 2010).

The second pathway involves the plant hormones auxin and CK. Local auxin response has been shown to trigger organ initiation at discrete sites in the SAM peripheral zone (e.g. Okada et al., 2001; Benková et al., 2003). Consistent with this, auxin response maxima and the polar auxin transport facilitator PIN1 are present in the Cardamine rachis directly corresponding to the sites of leaflet outgrowth. By contrast, these features are not present in the petiole of Arabidopsis, thereby indicating the role of auxin in leaflet promotion. Consistent with this, lossof-function pin1 in Cardamine or applying the auxin transport inhibitor 1-N- naphthylphthalamic acid (NPA) to wild type Cardamine leaves results in partial or complete transformation of compound leaves to simple leaves (Barkoulas et al., 2008). Auxin-dependent signaling may also regulate leaflet formation since the combined loss of AUXIN-RESISTANT1 (AXR1) and AS1 activities leads to the formation of leaflets at the junction between the blade and petiole, mimicking the compound leaf form (Hay et al., 2006b). AXRI encodes a subunit of the RUB1 ubiquitin-activating enzyme that promotes $\mathrm{SCF}^{\mathrm{TIR}} \mathrm{E} 3$ ubiquitin ligase activity and auxindependent gene expression (Leyser et al., 1993). AXR1 and AS1 activities converge to repress 
$B P$ expression in leaves, suggesting that they facilitate simple leaf formation by regulating KNOX1 meristematic genes in leaves (Hay et al., 2006b). Although BOP1/2 function redundantly with AS1 and AS2 in leaf patterning (Ha et al., 2003; 2007; Chapter 3) the combined mutation of bop1 bop 2 and axr1 does not lead to ectopic leaflet formation (Musa, 2010). Therefore, it remains to be seen if $\mathrm{BOP} 1 / 2$ negatively regulates auxin accumulation to promote simple leaf formation. CK is also involved in simple-compound leaf patterning. Up or down regulation of $\mathrm{CK}$ biosynthesis results in more or less complicated compound leaves, respectively, in tomato plants (Shani et al., 2010). As auxin and KNOXI genes are involved in compound leaf formation, how does $\mathrm{CK}$ interact with them in compound leaf formation? Shani and co-workers (2010) showed that the effect of CK on leaf complexity correlates with auxin accumulation and that $\mathrm{KNOX} 1$ acts upstream of $\mathrm{CK}$ in leaf patterning.

A third pathway involves the Arabidopsis organ boundary CUC genes. Hasson et al. (2011) showed that ectopic expression of CUCl triggers ectopic leaflet formation on the petiole, transforming simple leaves to compound leaves. They also showed that the ectopic expression of $C U C 1$ results in ectopic expression of $S T M$ and $B P$ but not $K N A T 2$ at the blade-petiole junction.

In summary, variation in leaf architecture contributes to the diversity of plants. Leaf initiation is a coordinated process that involves the down regulation of KNOXI genes and up regulation of $P I N 1, A S 1 / 2$, and $B O P 1 / 2$ in leaf primordia. Two aspects of leaf patterning occur after leaf initiation: adaxial-abaxial patterning and proximal-distal patterning. Adaxial identity is specified by Class U HD-ZIP transcription factors, as well as BOP1/2 and AS2, whereas abaxial identity is specified by $\mathrm{YAB}$ and KAN transcription factors. Proximal-distal patterning is controlled by KNOX1 homeodomain proteins, the plant hormones auxin and $\mathrm{CK}$, and the organ boundary gene CUC1. In Arabidopsis, the combined activities of BOP1/2, AS1, AS2, and AXR1 
maintain repression of $K N O X 1$ genes in leaves to control leaf shape. Studies in pea show that STAMINA PISTILLOIDA (STP), a homolog of Arabidopsis UNUSUAL FLORAL ORGANS (UFO), and UNIFOLIATA (UNI), the homolog of Arabidopsis LEAFY (LFY), are required for pea compound leaf development (Hofer et al., 1997; Taylor et al., 2001). UFO and LFY promote floral meristem identity and floral organ identity in Arabidopsis, however, a role for UFO and LFY in Arabidopsis simple leaf development has not been found.

\subsection{Reproductive plant architecture}

In response to appropriate endogenous and environmental cues, Arabidopsis plants transition to reproductive development during which time the architecture of the plant changes dramatically. Modules produced during by the apical meristem are elaborated differently during reproductive development. In Arabidopsis, internodes are not elongated until floral induction. In the firstinflorescence stage, cauline leaves with axillary meristems that differentiate as secondary inflorescences (an indeterminate shoot-type) are produced. In the second-inflorescence stage, leaf development is fully repressed and axillary meristems differentiate as flowers (a determinate shoot-type). Variables that contribute to the diversity of inflorescence architecture include the fate and determinacy of the axillary meristem, internode length, the phyllotaxy and the orientation of the lateral structures (inflorescences and flowers). These various architectural traits are controlled by the interacting activities of several classes of regulators in Arabidopsis that are explained in the sections that follow.

\subsubsection{The decision to flower}

The transition from vegetative to reproductive development requires the SAM to adopt an IM fate, such that inflorescences and flowers are produced at the expense of leaves. Therefore, acquiring an IM fate is the first step of reproductive development. The transition from SAM to 
IM identity is tightly controlled by several pathways: the long-day photoperiod pathway, the vernalization pathway, the gibberellin (GA) pathway, the thermosensory pathway, and the autonomous pathway (reviewed in Jack, 2004; Liu et al., 2009a; Figure 1.6). In Arabidopsis, the long-day photoperiod pathway promotes flowering whereas GA accelerates flowering especially under short-day conditions. The vernalization pathway facilitates the response to cold temperature induction whereas the autonomous pathway regulates flowering by perceiving endogenous signals related to the age of the plant. Input from these pathways is integrated by a small set of genes (termed floral integrators) that include FLOWERING LOCUS T (FT), SUPPRESSOR OF OVEREXPRESSION OF CONSTANSI (SOC1) and LEAFY (LFY), which are expressed at low levels during vegetative development but strongly up regulated in the SAM upon floral induction (Parcy, 2005). The up regulation of these genes in shoot apices directs the formation of an inflorescence and causes the activation of floral-meristem identity (FMI) genes in lateral organ primordia so as to confer floral fate. In Arabidopsis, the two key FMI regulators are $L F Y$ and APETALAI (API). These genes have several important functions: a) to promote the expression of genes that specify floral meristem fate, $b$ ) to repress the continued expression of genes that confer inflorescence meristem identity, and c) to activate genes that specify floralorgan identity (FOI). These activities are required to ensure the specification of floral meristems that are determinate and terminate after the production of four whorls of floral organs (sepals, petals, stamens, and carpels).

\subsubsection{Internode elongation and pedicel orientation}

In Arabidopsis, the three-amino-acid loop-extension (TALE) super family of homeodomain proteins consists of two structurally and functionally related subclasses: KNOX homeodomain proteins and BEL1-like (BELL) homeodomain proteins (Hamant and Pautot, 2010). Members 
from these two subclasses such as BP from the KNOX subclass and PENNYWISE (PNY) (also called BELLRINGER, REPLUMLESS and VAAMANA) from the BELL subclass control internode length and pedicel orientation (Douglas et al., 2002; Venglat et al., 2002; Byrne et al., 2003; Smith and Hake, 2003). Molecular and genetic analyses have revealed both redundant and antagonistic interactions among them. During Arabidopsis vegetative development, STM, BP, KNAT6, PNY, PNF, and ATH1 function redundantly to establish and maintain the SAM (Byrne et al., 2000; 2002; 2003; Ori et al., 2000; Belles-Boix et al., 2006). However, during reproductive development, KNAT2, KNAT6, and ATH1 function antagonistically to BP and PNY in regulating internode elongation and pedicel orientation (Ragni et al., 2008).

\subsubsection{BP and PNY-PNF promote internode formation}

In addition to their roles in establishing and maintaining the SAM, BP and PNY-PNF homeodomain proteins regulate inflorescence architecture. Loss-of-function $b p$ mutants display reduced internode length and horizontal or downward-pointing siliques (Douglas et al., 2002; Venglat et al., 2002). In contrast, loss-of-function pny mutants display internodes of irregular length and clusters of siliques (Byrne et al., 2003; Smith and Hake, 2003). Strikingly, the inflorescence of $b p$ pny double mutants is extremely short compared to its parents, indicating redundant roles for BP and PNY in promoting internode elongation (Smith and Hake, 2003; Ragni et al., 2008). Mutations in $P N F$, a gene closely related to $P N Y$, do not obviously affect internode elongation but the pny pnf double mutant fails to produce an inflorescence and continues to produce leaves indefinitely (Smith et al., 2004). This suggests a redundant role for PNY and PNF in co-coordinating internode elongation and the decision to flower.

Since there are synergistic interactions between BP, PNY, and PNF, what are the mechanisms that explain the functions of these proteins in internode patterning? This question is 
only partly answered. Consistent with the overlapping activities of these genes, $B P$ and $P N Y$ are expressed in the cortex of internodes and pedicels whereas $P N F$ is only expressed in the IM (Lincoln et al., 1994; Byrne et al., 2003; Smith et al., 2004). Yeast-two-hybrid assays have revealed heterodimers of $\mathrm{BP} / \mathrm{PNY}$ and $\mathrm{BP} / \mathrm{PNF}$ providing a partial explanation for redundancy in their activities (Kanrar et al., 2006).

In addition to promoting internode elongation, BP and PNY affect cell differentiation in the internodes. Histological analyses of pny and $b p$ stem cross-sections reveal an abnormal pattern of cell differentiation and lignin accumulation, albeit in slightly different patterns for the two mutants (Venglat et al., 2002; Mele et al., 2003; Smith and Hake, 2003). In bp mutants, lignin is ectopically deposited in discrete regions of the stem epidermis and cortex. In the epidermis, lignin is ectopically deposited in stripes of abnormally-differentiated epidermal tissue that extend below nodes, whereas in the cortex, lignin is prematurely deposited in intrafascicular bundles indicating the accelerated differentiation of secondary xylem (Douglas et al., 2002; Mele et al., 2003; Venglat et al., 2002). In pny stems, there are a greater number of vascular bundles resulting in a continuous ring of lignified stem cortex (Smith and Hake, 2003). Further, DNA microarray and in vitro binding studies performed by Mele et al. (2003) indicate that $B P$ is a direct negative regulator of lignin biosynthetic genes. Similar studies have not been done with $P N Y$. One interpretation of these data is that $B P$ and $P N Y$ in the stem cortex serve to coordinate internode elongation with the developmental timing of xylem differentiation.

\subsubsection{KNAT2-KNAT6 and ATH1 inhibit internode elongation}

In the vegetative SAM, STM, BP, KNAT6, PNY, PNF, and ATH1 function redundantly to maintain the SAM. Surprisingly, KNAT2, KNAT6, and ATH1 function antagonistically to BP and PNY in the inflorescence. In WT inflorescences, reporter gene studies show that KNAT2 and 
KNAT6 expression domains are restricted to the pedicel axil. However, in $b p$ and pny mutants, the expression domains of both genes expand to stems and pedicels (Ragni et al., 2008). Not surprisingly, knat 2 knat6 double mutations rescue internode length and pedicel orientation in $b p$ and pny single mutants and in bp pny double mutants (Ragni et al., 2008). Mutations in KNAT6 were more effective than mutations in $K N A T 2$ in rescue of $b p$ and $p n y$ defects indicating unequal requirements for these genes (Ragni et al., 2008). These data indicate that BP-PNY and KNAT2KNAT6 function antagonistically to regulate growth patterns in the inflorescence stem. It is not yet known if knat 2 knat 6 can rescue the lignification pattern of $b p$ and pny or if knat 2 knat6 mutations rescue the pny pnf block in inflorescence formation.

Mutation of the BELL homeodomain protein encoded by $A T H 1$ also fully rescues pny inflorescence defects and at least partially restores the pny pnf bolting defect when mutated (Rutjens et al., 2009) indicating antagonistic functions for ATH1 and PNY/PNF in the inflorescence. It is not known if $A T H 1$ mutations also rescue $b p$ inflorescence defects. How these factors function antagonistically is not clear but earlier characterization of $A T H 1$ has shown that it promotes the expression of FLOWERING LOCUS C (FLC), a potent repressor of flowering (Proveniers et al., 2007; Liu et al., 2009a). Thus, regulation of ATH1 may provide an important link between coordination of internode elongation and flowering that has not yet been investigated. This idea is discussed further below.

\subsubsection{A role for AS2 in inflorescence patterning}

AS1 and AS2 have been shown to repress $B P$ expression during leaf development (Byrne et al., 2000; Ori et al., 2000; Semiarti et al., 2001; Iwakawa et al., 2002). Consistent with this, ectopic expression of $A S 1$ and $A S 2$ results in reduced internode length and downward pointing siliques, resembling loss-of-function $b p$ mutants (Lin et al., 2003; Xu et al., 2003). Remarkably, over- 
expression of $B O P 1$ or $B O P 2$ also causes internode length and pedicel orientation defects (Norberg et al., 2005; Ha et al., 2007) similar to loss-of-function $b p$ mutants or overexpression of AS1/2. However, inactivation of $A S 2$ in $35 S:: B O P 1$ plants only slightly rescues its inflorescence architecture defects (Ha et al., 2007) indicating that the effect of BOP1/2 on inflorescence architecture is largely independent of AS2.

Taken together, the length of internodes and orientation of pedicels in inflorescences is regulated by the activities of KNOX and BELL homeodomain proteins. BP, PNY and PNF function redundantly to promote internode elongation and proper pedicel orientation, whereas KNAT2, KNAT6, and ATH1 have opposing functions. How these groups of regulators exert their antagonistic functions remain to be elucidated. Gain-of-function AS1/2 and BOP1/2 also perturb inflorescence architecture. However, in the inflorescence, BOP $1 / 2$ does not primarily exert its effect through AS2.

\subsubsection{Coordination of internode elongation and flowering}

In many plant species, including Arabidopsis, the switch to flowering and elongation of the first internode are tightly linked. Several lines of data suggest that coordination of these two events requires PNY and PNF activities (Smith et al., 2004; Kanrar et al., 2008). As explained above, pny pnf double mutants do not flower in response to appropriate inductive signals (Smith et al., 2004). While the transcripts of several floral integrators accumulate normally in pny pnf apices, $L F Y$ and $A P 1$ transcripts (FMI genes) do not accumulate. While overexpression of $L F Y$ rescues pny pnf flowering, overexpression of the floral integrator $F T$ does not, suggesting that FT requires the activities of PNY and PNF to be functional (Kanrar et al., 2008). Mutation of the BELL-like gene $A T H l$ partially rescues the pny pnf non-flowering phenotype (Rutjens et al., 2009) suggesting that its misexpression in pny pnf mutants may interfere with floral evocation, 
but this remains to be tested. Plants overexpressing $A T H 1$ are very late flowering due to up regulation of the floral repressor gene FLOWERING LOCUS C (FLC) (Proveniers et al., 2007; Liu et al., 2009a), suggesting a link between flowering and internode elongation. PNY and PNF also regulate flower identity together with LFY, UFO, and WUS (Yu et al., 2009). Identification of direct targets of the TALE proteins, as with the case of AGAMOUS $(A G)$ for PNY (Bao et al., 2004), would help us to understand the mechanism that coordinates the internode elongation and flowering.

\subsubsection{Specification of floral meristem identity}

Upon floral induction, the IM produces secondary inflorescences and then flowers (Figure 1.1). In Arabidopsis, the architecture of a secondary inflorescence differs from a flower in three aspects. First, a secondary inflorescence has an indeterminate meristem with the potential to produce numerous flowers, whereas flower meristems (FMs) are determinate and produce a set number of floral organs before the stem-cell population is terminated. Second, inflorescence meristems produce flowers arranged in a spiral pattern with internode elongation between organs, whereas floral meristems produce floral organs arranged in a whorled pattern without internode elongation between organs. Third, a secondary inflorescence has a cauline leaf at its base, whereas an Arabidopsis flower is bractless (Huala and Sussex, 1992; Weigel et al., 1992; Levin et al., 1995).

The morphological changes that occur during Arabidopsis floral development can be

divided into 12 stages according to a series of landmark events (Smyth et al., 1990). Floral primordia are generally considered as floral meristems (FM) as they produce floral organs. Stage 0 FMs are morphologically invisible, but can be distinguished from other cells in the IM by the expression of marker genes such as $L F Y$ (Hempel et al., 1997). Stage 1 FMs as seen as lateral 
bulges on the flanks of the IM. Stage 2 FMs are separated from the IM by a groove and are enlarged into rounded structures. In Stage $3 \mathrm{FMs}$, the pedicel begins to elongate and sepal primordia develop on the meristem periphery. They develop into stage 4 FMs when the sepal primordia grow to cover the FM. After stage 4, stamen and petal primordia develop, while the inner most cells in the FM gives rise to the carpel primordia.

As explained in the introduction to this section, the expression of FMI markers such as $L F Y$ and $A P 1$ in lateral organ primordia confers floral fate and they activate a chain of events resulting in the production of a flower (Irish and Sussex, 1991; Weigel et al., 1992; Bowman et al., 1993; reviewed in Krizek and Fletcher, 2005). Mutations or combinations of mutations in FMI genes cause a transition to indeterminacy, flowers with bracts or internode elongation between successive floral organs, which are all characteristics of secondary inflorescences (Parcy, 2005, Liu et al., 2009a).

\subsubsection{LFY}

LFY is viewed as the central regulator of FMI. However, its expression is dependent on input from several flowering time pathways, so it also behaves as a floral integrator (Weigel et al., 1992; Parcy 2005, Liu et al., 2009a). Thus far, SOC1 is the only transcriptional regulator that is found to bind to the $L F Y$ promoter directly (Lee et al., 2008). SOCl is directed into the nucleus by AGL24, another MADS-box (for MCM1-AGGAMOUS-DEEFICIENS-SEERUM RESPONSE FACTOR) protein with a domain of expression overlapping with SOC1. Based on these observations, Lee et al. (2008) suggested that SOC1-AGL24 heterodimers directly activate $L F Y$ expression in vivo. The floral integrator FT and its co-factor FLOWERING LOCUS D (FD) also activate $L F Y$ expression, but this activation could be indirect since FT/FD activates $S O C I$ (Abe et al., 2005) and SOC1 directly promotes $L F Y$ expression (Lee et al., 2008). 
$L F Y$ transcripts are very low in vegetative leaf primordia but accumulate to high levels in floral primordia from the earliest stages (Blazquez et al., 1997; Hempel et al., 1997). LFY expression first occurs in P0 primordia. At stage 3, $L F Y$ expression concentrates in the dome of the FM where its activity turns on genes that confer floral organ identity (Weigel et al., 1992; Hempel et al., 1997; Liu et al., 2009a).

Consistent with its role as a master regulator of FMI, mutations in $L F Y$ cause the complete conversion of early-arising flowers into inflorescences. In strong lfy mutants, more flowers are transformed into secondary inflorescences than in weak alleles, and late-arising flowers retain partial characteristics of secondary inflorescences (e.g. secondary flowers arise in the axils of the outer floral organs, floral organs are separated by internodes, and flowers are subtended by bracts) (Schultz and Haughn, 1991; Huala and Sussex, 1992; Weigel et al., 1992). Once $L F Y$ is activated in floral primordia, it directs the activation of additional FMI genes including the MADS-domain transcription factors encoded by APl and CAULIFLOWER (CAL) and the homeodomain-leucine zipper protein encoded by $L M I 1$. These factors reinforce $L F Y$ expression in a feed-forward loop that makes the switch from inflorescence to flower production unidirectional (Parcy et al., 1998; Wagner et al., 1999; Ferrandiz et al., 2000; Williams et al., 2004; Saddic et al., 2006).

\subsubsection{AP1}

$A P 1$ is first activated in stage $1 \mathrm{FMs}$ and is the earliest known marker of commitment to floral fate (Hempel et al., 1997). LFY is a direct regulator of AP1 (Parcy et al., 1998; Wagner et al., 1999) but its expression is also directed by a complex of FT/FD (Abe et al., 2005; Wigge et al., 2005) and by SQUAMOSA PROMOTER BINDING PROTEIN-LIKE (SPL) proteins that control age-related flowering time (Wang et al., 2009; Yamaguchi et al., 2009). 
Unlike $L F Y, A P 1$ is not expressed in vegetative tissues, nor does it appear in the floral meristem until stage 1 (Mandel et al., 1992; Bowman et al., 1993; Hempel et al., 1997). In apl-1 mutants, secondary FMs arise in the axils of the first-whorl floral organs and this pattern is repeated several times, resulting in highly branched floral structures (Irish and Sussex, 1990; Bowman et al., 1993). These structures represent the partial conversion of flowers to inflorescences (indeterminate shoots because the FMs that used to give rise to a single flower now produce several flowers). When apl-1 is added to strong or weak alleles of $l f y$, the difference between strong and weak alleles of lfy largely disappears and the later-arising flowers are more shoot-like than in single mutants, indicating redundant roles for $L F Y$ and $A P 1$ in promoting FMI (Huala and Sussex, 1992; Weigel et al., 1992).

\subsubsection{Inflorescence meristem identity genes}

LFY and AP1 activities are required to block the continued expression of inflorescence meristem-identity genes in the floral meristem so that shoots become determinate and develop as flowers (Yu et al., 2004; Liu et al., 2007; 2009b). There are three main IM identity genes in Arabidopsis that encode MADS-domain transcription factors: SOC1, AGL24, and SHORT VEGETATIVE PHASE (SVP). All three genes are expressed in the IM and early FMs (stages 02) but the continued expression of any one of these genes in FMs partially transforms flowers into shoots with inflorescence-like characteristics, similar to mutation of lfy or apl-1 (Irish and Sussex, 1991; Weigel et al., 1992; Yu et al., 2004; Liu et al., 2007; 2009a). Consistent with this, loss-of-function agl24 is sufficient to rescue floral branching in ap1-1 mutants. Chromatin immunoprecipitation experiments suggest that $A G L 24, S O C 1$, and $S V P$ are directly repressed by AP1 whereas repression by LFY is probably indirect (Yu et al., 2004; Liu et al. 2007; Gregis et al., 2008). Paradoxically, in the early stages of FM development (stages 1 and 2) SOC1, SVP, 
and AGL24 expression in the FM promotes FMI (Samach et al., 2000; Yu et al., 2002; Michaels et al., 2003; Gregis et al., 2008). Several lines of evidence support this idea. First, SOCl and AGL24 contribute to $L F Y$ up regulation (Lee et al., 2008), Second, apl agl24 svp triple mutants develop a cauliflower-like apex that produces shoots in place of flowers, indicating that mutations in agl24 and svp enhance ap1-1 FMI defects (Gregis et al., 2008), similar to apl cal mutants that are also compromised for FMI (Ferrándiz et al., 2000).

In summary, LFY and AP1 are the master regulators of FMI. This task requires both the activation of secondary FMI genes and the repression of IM identity genes. Both activities are essential for the maintenance of FM fate so that flowers have a determinate structure.

\subsubsection{Floral patterning}

Floral organs begin to differentiate at floral stage 3. Sepals are seen to emerge first on the edges of the FM, followed by petal and stamen primordia. The FM is consumed by the formation of two central carpels that fuse to form the gynecium (Smyth et al. 1990). In contrast to floral primordia that arise from the IM in a spiral pattern, floral organ primordia are generated in concentric whorls (Smyth et al., 1990). The establishment of FMI and promotion of FOI are consecutive events, and some genes, such as $L F Y$ and $A P 1$, play roles in both processes (Irish and Sussex, 1990; Schultz and Haughn, 1991; 1993; Weigel, et al., 1992).

Our detailed understanding of how floral organs are patterned started with the characterization of mutants in which floral organs developed normally, but in inappropriate whorls of the flower (Bowman et al., 1991; Coen and Meyerowitz, 1991). It is now known that floral organ identity is determined by the overlapping activities of four classes of homeotic genes termed A, B, C, and E (Figure 1.7). With one exception, all of these genes encode MADS domain transcription factors (Krizek and Fletcher, 2005). According the ABCE model, A-class 
genes (e.g. $A P 1$ and $A P 2$ ) are expressed in the first whorl and specify sepals. In the second whorl, A-class and B-class genes (e.g. PISTILLATA $(P I)$ and AP3) are co-expressed and their products combine to specify petals. In the third whorl, B-class and C-class genes (e.g. AGAMOUS $(A G)$ ) are co-expressed and their products combine to specify stamens. In the fourth whorl, only Cclass genes are expressed and carpels are specified. The Class E genes (SEPALLATA1-4) were discovered later as a result of the Arabidopsis genome sequencing project. These genes function redundantly, are expressed in all whorls, and are required for the specification of all four organ types (Pelaz et al., 2000; Honma et al., 2001; Ditta et al., 2004). To account for the mutant phenotypes observed, the model also stipulates that A-class and C-class activities are mutually exclusive and repress each other. Consistent with this, in A-class mutants, C-class activity expands into all whorls, such that sepals are replaced by carpels and petals by stamens. Similarly, in C-class mutants, A-class activity expands into all whorls, such that carpels are replaced by sepals and stamens by petals. In C-class mutants, the flowers also become indeterminate because there is no mechanism to extinguish the stem-cell population and the production of sepals and petals continues indefinitely (Lenhard et al., 2001; Lohmann et al., 2001; Lohmann and Weigel, 2002).

LFY plays a central role in promoting FOI in all four whorls because its activity turns on the expression of key A-Class, B-Class, and C-Class genes in the FM. The A-class gene $A P 1$ is expressed in the perianth (sepal and petal) whorls of the flower and is a direct target of LFY activation (Parcy et al., 1998; Wagner et al., 1999). The A-class gene AP2 is also expressed in the outer floral whorls but its spatial domain of expression is determined post-transcriptionally by miR172 (Wollmann et al., 2010; Chen, 2004).

The B-class gene $A P 3$ is directly activated by LFY in the petal and stamen whorls but in 
this case, LFY requires the activity of an F-box co-factor called UNUSUAL FLORAL ORGANS (UFO). Most F-box proteins form the substrate adaptor component of an SCF complex which acts an E3 ubiquitin ligase to mark targets with polyubiquitin so that they are recognized the $26 \mathrm{~S}$ proteosome and degraded (Deshaies, 1999). The requirement for UFO is surprising since the LFY protein has an activation domain and does not require a co-factor to turn on AP1 (Parcy et al., 1998; Wagner et al., 1999). Nevertheless, several lines of evidence suggest that LFY and UFO activities are both needed to activate $A P 3$ expression. First, flowers of strong lfy and $u f o$ mutants do not have petals and stamens (Weigel et al., 1992; Wilkinson and Haughn, 1995), similar to that of ap3 mutant. Consistent with this, AP3 expression is significantly downregulated in these mutants (Weigel and Meyerowitz, 1993; Levin and Meyerowitz, 1995). Second, over expression of $A P 3$ and $P I$ can partially restore the petals and stamens in lfy and ufo mutants and Lamb et al. (2002) demonstrated that LFY binds directly to the AP3 promoter. Work by Chae et al. (2008) found that the LFY-UFO complex directly binds to the AP3 promoter, suggesting that UFO is a co-factor for LFY in activating AP3. The other B-class gene $P I$ is expressed at the same domain as $A P 3$ and functions as a heterodimer with AP3 in floral patterning (Riechmann et al., 1996; Sundstrom et al., 2006).

The C-class gene $A G$ is expressed in the central dome of the FM beginning at stage 3 and specifies stamen and carpel identity. LFY, together with the meristem factor WUS, are the primary activators of $A G$. Both LFY and WUS have binding sites in the $A G$ second intron, which is the main regulatory region for $A G$ (Busch et al., 1999). WUS is co-expressed with AG from floral stage 3 to stage 5 for this function but it is turned off from floral stage 6 by AG to ensure that the stem-cell population in the FM terminates (Lenhard et al., 2001; Lohmann et al., 2001). 
The bZIP transcription factor PERIANTHIA (PAN) expressed in IMs and FMs also activates $A G$ expression directly (Das et al., 2009; Meier et al., 2009).

The SEP1-4 genes are expressed in overlapping domains that encompass all four whorls of the flowers. They function in association with Class A, B, and C genes to specify FOI (Krizek and Fletcher, 2005). Several lines of evidence support this model. First, sep1 sep2 sep 3 sep 4 quadruple mutants produce flowers with four whorls of leaf-like organs, resembling the phenotype of $a b c$ mutants (Pelaz et al., 2000; Ditta et al., 2004). Second, the ectopic expression of B-class and $S E P$ genes together is required for the successful conversion of leaves into petals (Honma and Goto, 2001; Pelaz et al., 2001b).

All of the floral homeotic genes with the exception of $A P 2$ encode MADS domain transcription factors, a type DNA-binding domain that is conserved among eukaryotes. How do these factors work in combination to specify FOI? Biochemical experiments show that MADS domain proteins form dimers in vitro and interact with a conserved DNA target sequence called a 'CArG box'. The B-class proteins AP3 and PI only bind to DNA as heterodimers, indicating that different complexes of MADS box proteins can bind selectively to sites in the promoters of their target genes (Reichmann et al., 1996; Honma and Goto, 2000). Large-scale interaction studies in yeast have led to the model that the assembly of MADS box proteins into higher order complexes containing four members might be the principal way in which $\mathrm{A}, \mathrm{B}, \mathrm{C}$, and E-class genes function combinatorially (Hongma and Goto, 2001; reviewed in Theissen, 2001). For example, AP1 interacts with SEP3, and AG interacts with SEP1, SEP2, and SEP3 (Pelaz et al., 2001a). Moreover, a large complex of AP3/PI/SEP3/AG was also discovered (Honma and Goto, 2001). SEP3 is proposed to act as a scaffold for the formation of quartet complexes of MADS box proteins (Immink et al., 2009). 
In summary, the overlapping activities of four classes of floral homeotic genes, termed A, B, C, and E specify Arabidopsis floral organ identity by forming multimeric complexes of four proteins that activate genes required for sepal, petal, stamen, and carpel differentiation (Coen and Meyerowitz, 1991; Krizek and Fletcher, 2005). The expression of several key A, B, and C class genes $(A P 1, A P 3$, and $A G)$ are directly dependent on LFY for their activation in specific domains of the FM (Busch et al., 1999; Wagner et al., 1999; Lamb et al., 2002) whereas the AP2 expression domain is determined by miR172 (Chen, 2004; Wollman et al., 2010). The E-class SEP genes are activated independently of LFY and play an important role as the "glue" that mediates the formation of MADS-domain protein "quartets" for the specification of FOI (Theissen, 2001; Pelaz et al., 2000; 2001b; Ditta et al., 2004; Immink et al., 2009).

\subsection{BOP signaling mechanism}

BOP1 and BOP2 belong to the NON-EXPRESSOR OF PATHOGENESIS-RELATED GENES1 (NPR1) family of proteins, all of which contain an N-terminal BTB/POZ (for Broad-Complex, Tramtrack, and Bric-a-Brac/POX virus and Zinc finger) domain and a C-terminal set of four ankyrin repeats (Cao et al., 1997; see Hepworth et al., 2005 for a phylogenetic tree of the six NPR1 family members in Arabidopsis). The BTB/POZ domain is found to interact with Cullin 3 proteins that target substrates for ubiquitin-mediated degradation whereas the ankyrin domain interacts with TGA (TGACG-motif binding) bZIP (basic leucine zipper) transcription factors (Hepworth et al., 2005). NPR1 is the founding member of the gene family, and it was identified by mutation as a central regulator of systemic acquired resistance (SAR) in plants (Cao et al., 1997). SAR is an induced immune response triggered by local infection that protects the entire plant against subsequent bacterial, fungal, and viral infection (Dong, 2004). BOP1/2 have no apparent function in plant disease resistance and instead regulate the architecture of leaves, 
inflorescence, fruits, and flowers (Hepworth et al., 2005; Norberg, et al., 2005; Ha et al., 2003; 2007; McKim et al., 2008). Genes or transcripts with homology to $B O P 1$ and $B O P 2$ are found in various plant species including moss, trees, dicots and monocots (McKim, 2009). In moss, BOP proteins promote cell differentiation (Saleh et al., 2011). These findings suggest that BOP proteins are ancient and conserved and play a fundamental role in controlling plant architecture and development. Because of the conserved domain structure of BOP1/2 and NPR1, studies pertaining to NPR1 serve as a paradigm for understanding how BOP1/2 might be regulated and how they might control transcription at the biochemical level.

Briefly, NPR1 promotion of SAR in plants is regulated primarily at the posttranscriptional level (Cao et al. 1998; Spoel et al. 2010). In response to challenge with pathogen, the plant hormone salicylic acid (SA) accumulates systemically in plants leading to changes in the intracellular redox potential of cells that convert NPR1 into an active monomer that is allocated to the nucleus. In the nucleus, NPR1 is recruited to the promoter of its target genes primarily through association with members of the TGA subclass of bZIP transcription factors thus activating plant defense (Zhang et al., 1999; Despres et al., 2000; Mou et al., 2003; reviewed in Dong, 2004). Evidence suggests that this basic mechanism may apply in part to BOP1 and BOP2. First, BOP1/2 also forms complexes in yeast with TGA bZIP family members, including TGA8/PAN that functions in the same pathway as BOP1/2 to control sepal number in flowers (Hepworth et al., 2005). Second, BOP1-GFP fusion proteins localize to both the cytoplasm and the nucleus of cells (Hepworth et al. 2005; Jun et al. 2010). Third, BOP1/2 function as transcriptional activators (Jun et al. 2010; Xu et al. 2010). However, it remains unclear if BOP1/2 nuclear localization, stability, transcriptional activation, and interactions with TGA factors are regulated by redox-dependent modification of Cys residues since only two of 
several Cys regulatory residues in NPR1 are conserved in BOP1 and BOP2 (Hepworth et al., 2005; reviewed in Spoel et al., 2010).

Analysis of NPR1 expression before and after SA treatment suggests that NPR1 activity is primarily posttranscriptionally regulated, as $N P R I$ is constitutively expressed and its transcripts are increased only two-fold after SA treatment (Cao et al., 1998). Kinkema and coworkers (2000) first found that nuclear localization of NPR1 is essential for its activity during SAR. Later on, Mou and co-workers (2003) found that NPR1 normally resides in the cytoplasm in oligomeric form, holding together by intermolecular disulfide bonds. Upon pathogen challenge or SA treatment, the cellular redox state changes, leading to the reduction of these disulfide bonds, and resulting in nuclear localization of monomeric NPR1 and activation of its target genes. These authors also showed that mutations of cysteine at Cys82 and Cys216 to Alanine in NPR1 lead to increased monomer accumulation, constitutive nuclear localization and expression of $P R I$ (a target gene) without induction, thereby confirming that monomerization of NPR1 mediates its nuclear localization and activity. However, the fact that plants treated with SA not only release NPR1 monomer but also accumulate NPR1 oligomer, and that removing reducing agent from NPR1 protein solutions results in the oligomer reformation, indicated that NPR1 undergoes conformational changes under different cellular environment (Tada et al., 2008).

Further molecular, genetic and biochemical analysis demonstrated that reversion of NPR1 monomers to oligomers is carried out through S-nitrosylation, probably on Cys156 (Tada et al., 2008). As such, S-nitrosoglutathione, a natural NO donor, can effectively reduce monomer NPR1 levels without changing the total NPR1. Moreover, the atgnsor l-3 mutant, which displays increased S-nitrosylation activity, cannot effectively induce nuclear localization of NPR1 and 
$P R I$ expression upon SA treatment, indicating that there is little or no NPR1 monomer available due to the oligomerization of NPR1 by S-nitrosylation (Tada et al., 2008). These findings confirm that NPR1 is oligomerized by S-nitrosylation.

Localization of NPR1 monomer in the nucleus is necessary but not sufficient for its transcriptional activity. Spoel and co-workers (2009) demonstrated that under non-inducing conditions the nuclear localized monomer NPR1 is degraded by the CUL3-mediated activity, preventing the costly activation of SAR. By contrast, during SAR, NPR1 is phosphorylated at Ser11 and Ser15 in the nucleus, and the phosphorylation of NPR1 stimulates its transcriptional activity. NPR1 has no DNA binding domain but has ankyrin repeats that interact with TGA transcription factors (Zhang et al., 1999; Despres et al., 2000). After SA treatment, NPR1 binds to TGA transcription factors (such as TGA2), forming a enhanceosome on the $P R 1$ promoter to activate the transcription of $P R 1$ (Zhang et al., 1999; Despres et al., 2000; Rochon et al., 2006; Pape et al., 2010).

In moss, $B O P$ expression is regulated post-transcriptionally by miRNA534, which controls the timing of juvenile-to-adult phase change (Saleh et al., 2011). It is possible that BOP is targeted by miRNAs in Arabidopsis since it has various effects on plant development, resembling the mutation of the miRNA targets AP2 (Chen et al., 2004) and SPL3/9 (Wang et al., 2009; Wu et al., 2009; Yamaguchi et al., 2009) but no such miRNAs have been identified for BOP in Arabidopsis (Saleh et al., 2010). Future work will need to address whether Cys residues in the BOP1 and BOP2 post-transcriptionally control BOP activity through oxidation-reduction, nitrosylation and/or phosphorylation to regulate association with DNA-binding partners or transcriptional activation potential. 


\subsection{Thesis rationale}

The overall goal of our work is to understand the mechanisms that control plant architecture. Our lab is interested in how two conserved BTB-ankyrin domain-containing transcriptional co-regulators, BOP1 and BOP2, control the architecture of Arabidopsis leaves, inflorescences and flowers (Ha et al., 2003; Hepworth et al., 2005; Norberg et al., 2005; Ha et al., 2007; McKim et al., 2008). These genes are expressed in initiating lateral organ primordia produced during both vegetative and reproductive phases of development and then partition to the boundaries that separate these organs from the meristem. In support of a central role for these genes in regulation of plant architecture, homologs are found in a wide variety of species (McKim, 2009) and they promote phase change in moss which is a lower plant (Saleh et al., 2010). Both loss and gain of BOP function in Arabidopsis cause dramatic changes in the patterning of leaves, inflorescences, and flowers (Ha et al., 2003; Hepworth et al., 2005; Norberg et al., 2005; Ha et al., 2007; McKim et al., 2008) suggesting that the temporal and spatial pattern of $B O P$ expression is an important determinant of plant architecture. While it is clear that these genes control plant architecture, how they function at the molecular level is poorly understood.

To address this gap in knowledge, I used a molecular genetics approach to address the role and mechanism of BOP $1 / 2$ activity in:

(1) floral meristems;

(2) leaves; and

(3) the node/internode junction of inflorescences

The results of these investigations form the basis of Chapters 2, 3, and 4 in my thesis. Chapter 5 provides a summary of these finding and future direction. Appendix A contains a glossary of the regulatory genes discussed in the thesis. Appendix B explains how double mutant analysis is 
used to infer genetic interactions between genes, a method that forms the basis of many of the experiments in this thesis. 


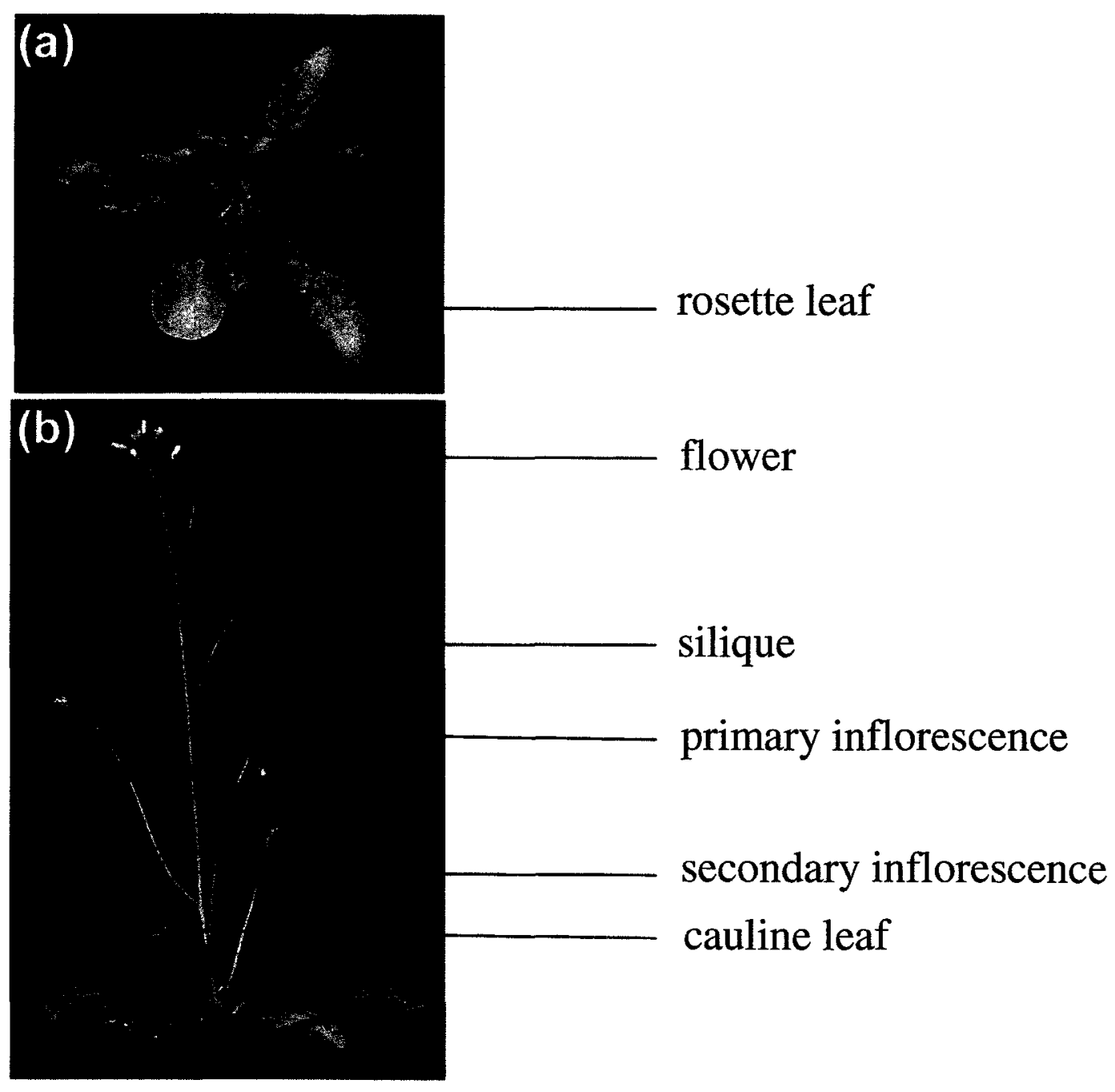

Figure 1.1 Arabidopsis thaliana developmental stages.

(a) Vegetative stage. Rosette leaves are produced without internode elongation. Axillary meristems may later develop in the axils of these leaves to produce a paraclade.

(b) Reproductive stage. In the first stage of reproductive development, cauline leaves form with secondary inflorescences in their axils. In the second stage of reproductive development, leaf development is repressed and axillary meristems develop as single flowers that mature into fruits (siliques). Internode elongation occurs during reproductive development so that lateral branches and flowers are evenly distributed along the primary inflorescence. 


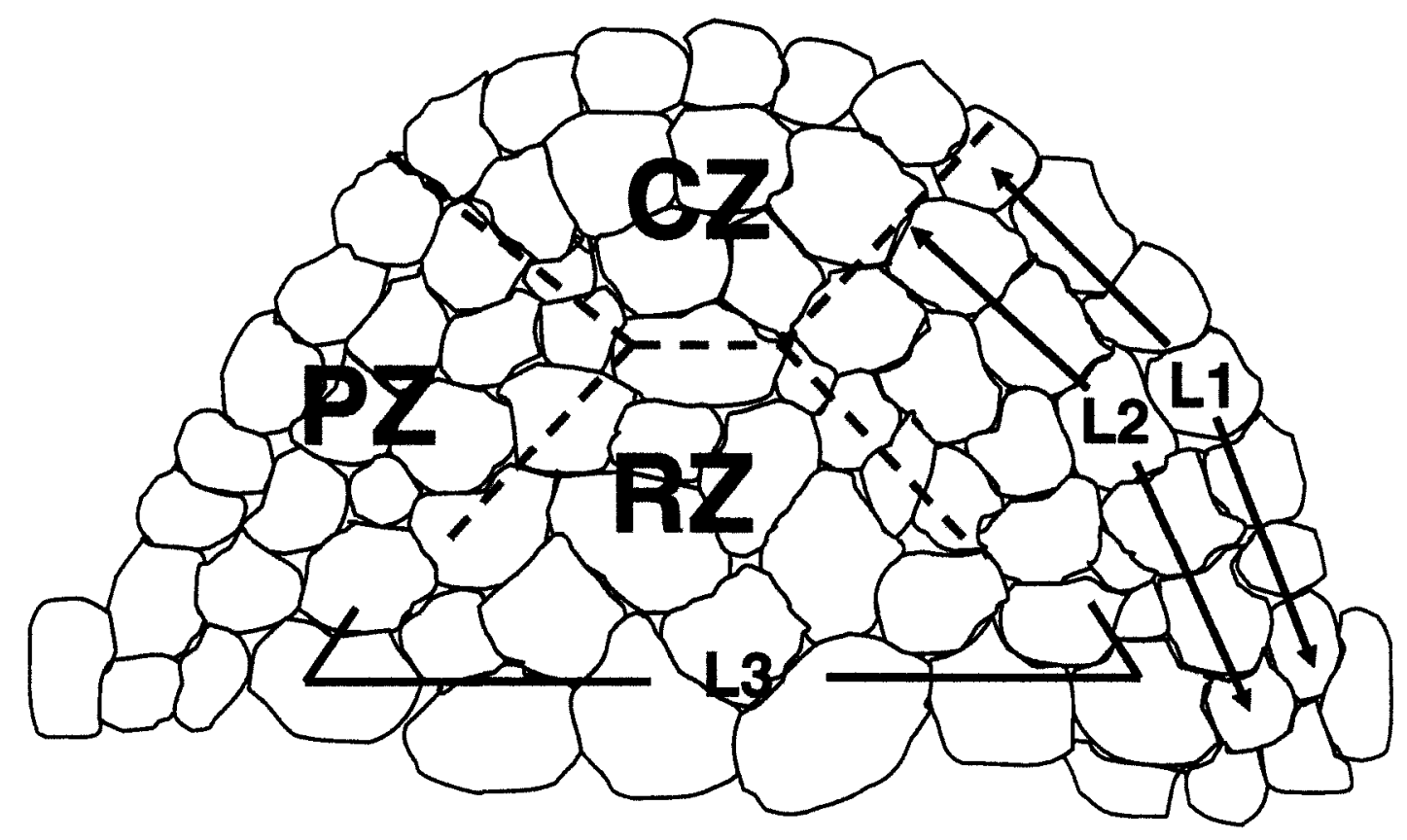

Figure 1.2 Organization of shoot apical meristem.

At the apex of the meristem, cells in the central zone (CZ) divide slowly to replenish the fund of pluripotent stem cells. The central zone is flanked by the peripheral zone (PZ), where organ primordia are initiated. The rib zone (RZ) lies beneath the central zone. The dashed lines indicate the boundaries between the different zones. It is not clear precisely how many cells constitute each region. Nevertheless, the outer $\mathrm{L} 1$ cell layer and the sub-epidermal L2 cell layer are a single cell thick, and the internal L3 cells lie beneath them. 


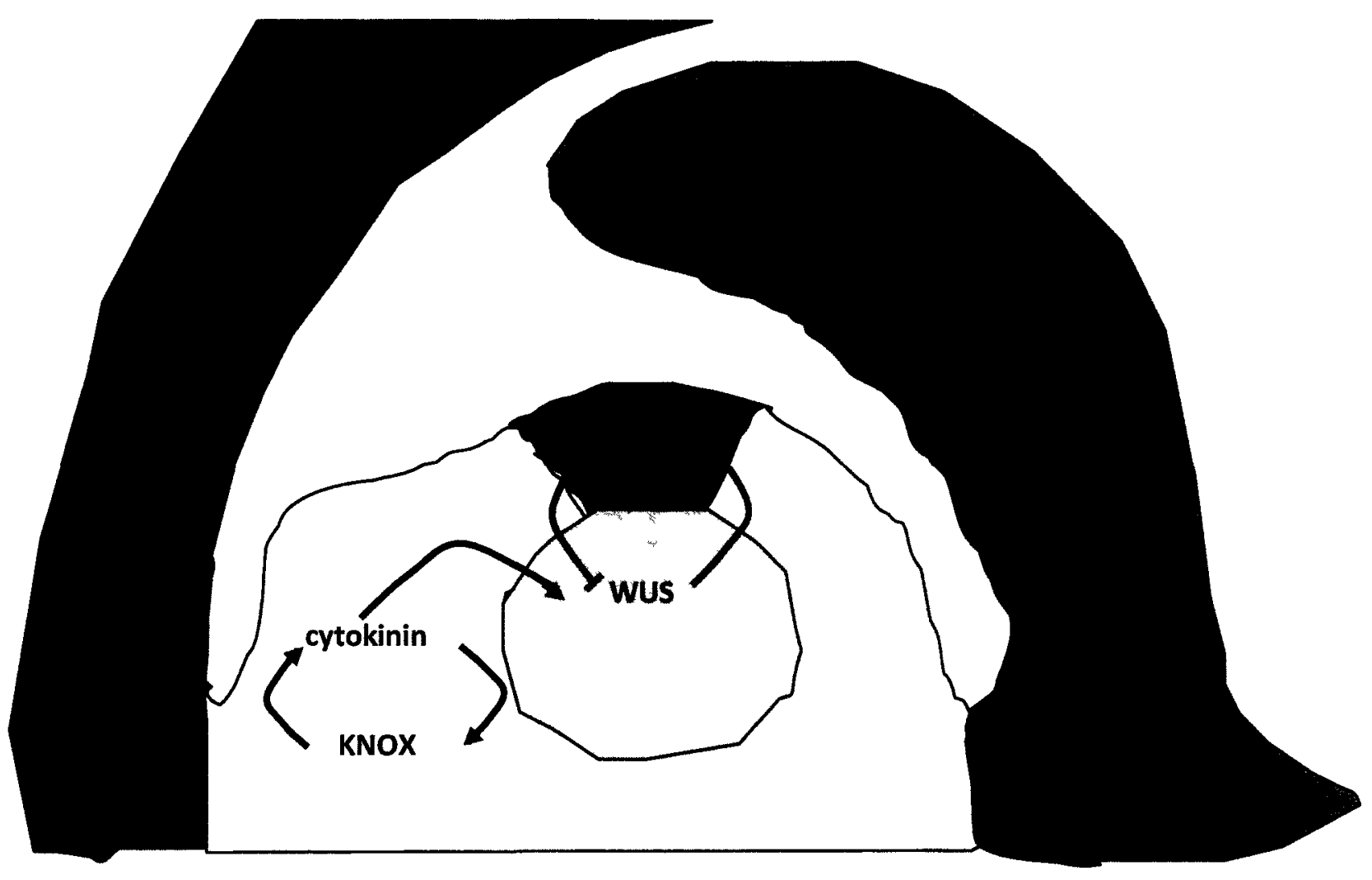

Figure 1.3 The shoot apical meristem is established and maintained by WUS-CLV3 signaling and KNOX-auxin/cytokinin signaling.

Diagram showing the expression domains of transcription factors involved in maintenance of the SAM and abaxial-adaxial leaf patterning. The transcription factor WUS is expressed in the organizing center induces the expression of a diffusible peptide CLV3 in the overlying central zone cells. CLV3 activates a signaling pathway that represses WUS expression, thereby creating a feed-back loop that maintains the size of the stem-cell population. Class I KNOX proteins modulate cytokinin levels which in turn activate KNOX and WUS expression. KAN and YAB activities promote abaxial leaf identity while HD-ZIPIII proteins in association with BOP1/2 and AS1-AS2 promote adaxial leaf identity. This figure is redrawn from Barton (2010) with permission from the publisher (Elsevier). 
(a)

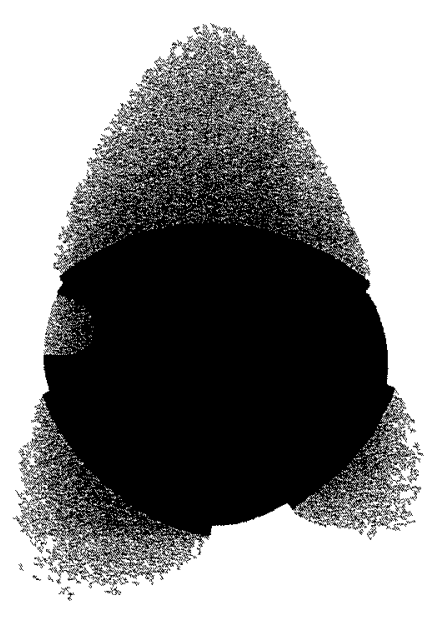

(b)

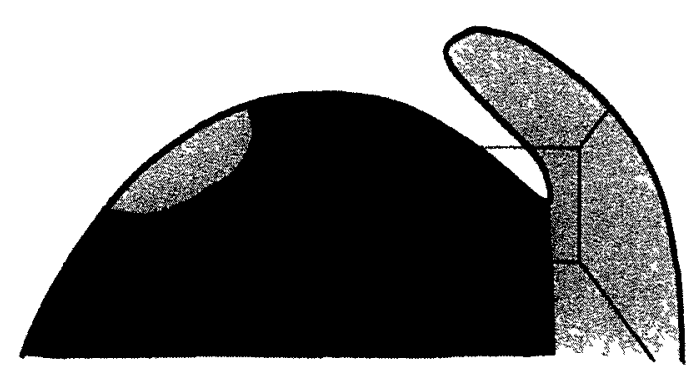

Figure 1.4 Schematic representations of the SAM and boundaries.

(a) Top view of the SAM. Red: meristematic region; blue: boundary domain; green: primordia.

CZ: Central Zone. Dotted lines indicate the stem cell domain.

(b) Side view of the SAM.

This figure is reprinted from Rast and Simon (2008) with permission of the publisher (Elsevier). 


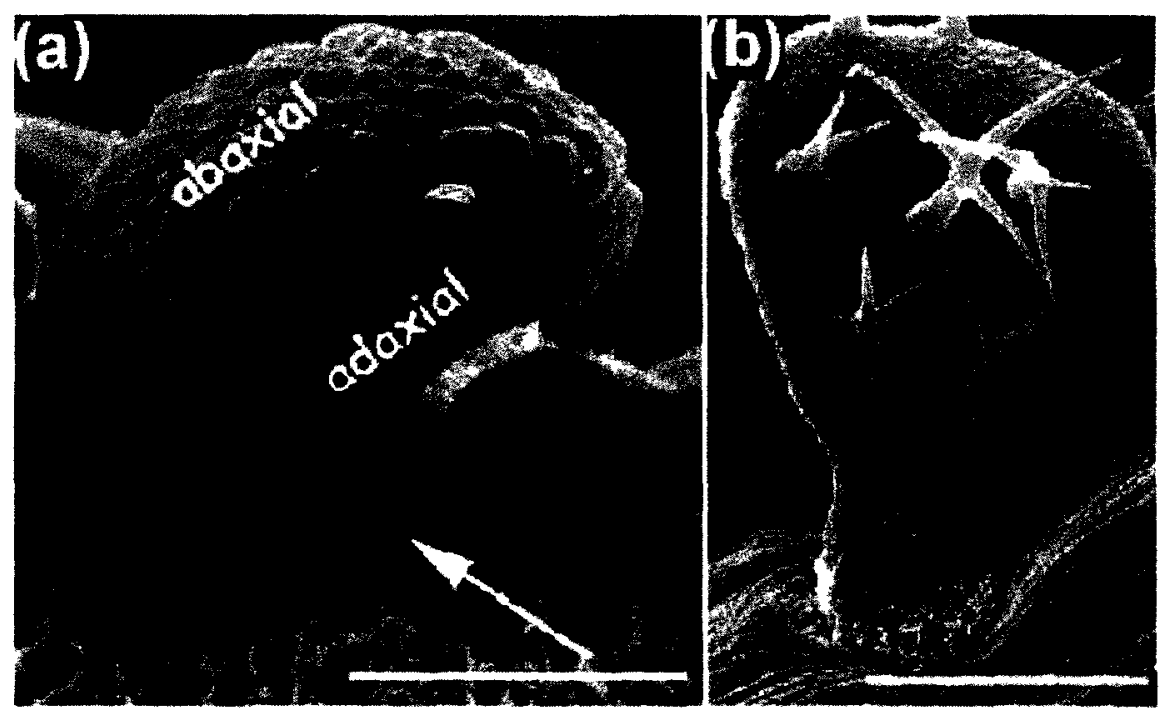

Figure 1.5 Adaxial-abaxial polarity in leaves.

SEM micrographs depicting:

(a) The adaxial and abaxial sides of an young Arabidopsis leaf. The adaxial side of the leaf is adjacent to the central SAM (arrow) whereas the opposite, abaxial, side of the leaf is farther from the SAM. Scale bar, $50 \mu \mathrm{m}$.

(b) A developing vegetative leaf. Trichomes are marker of adaxial leaf identity in early leaves. Scale bar, $250 \mu \mathrm{m}$.

This figure is reprinted from Byrne (2006) with permission of the publisher (PLoS Genetics). 


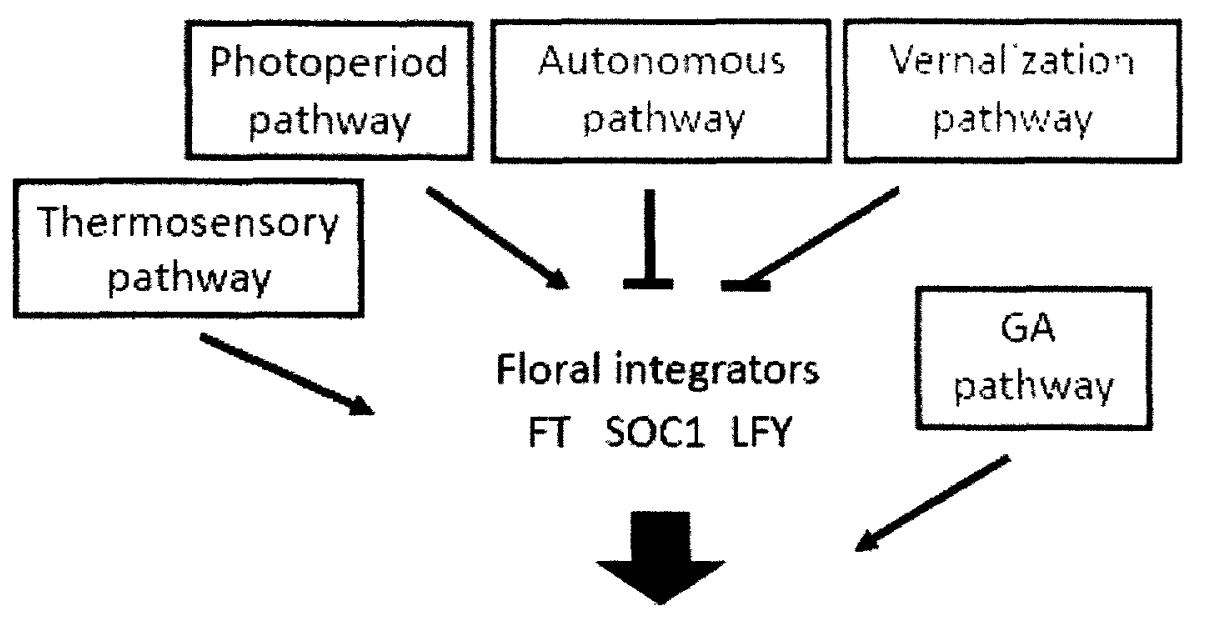

Floral meristem
dentity (FMI) factors
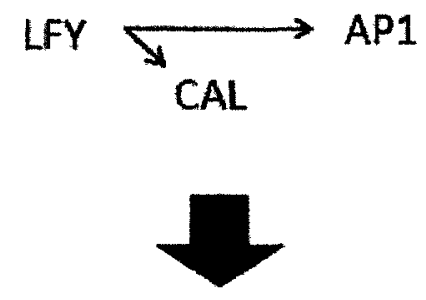

Floral organ identity (FOI) genes

Figure 1.6 Simplified diagram showing the pathways that control FMI.

Positive and negative inputs from five different flowering-time pathways converge to regulate the expression of a small number of genes with floral integrator activity. Upregulation of these genes in shoot apices promotes inflorescence meristem (IM) identity and the activation of floralmeristem identity (FMI) genes in lateral organ primordia. FMI genes in turn promote floral fate by turning on the expression of three overlapping sets of genes that combinatorially specify floral-organ identity (FOI) leading to the formation of flowers. 


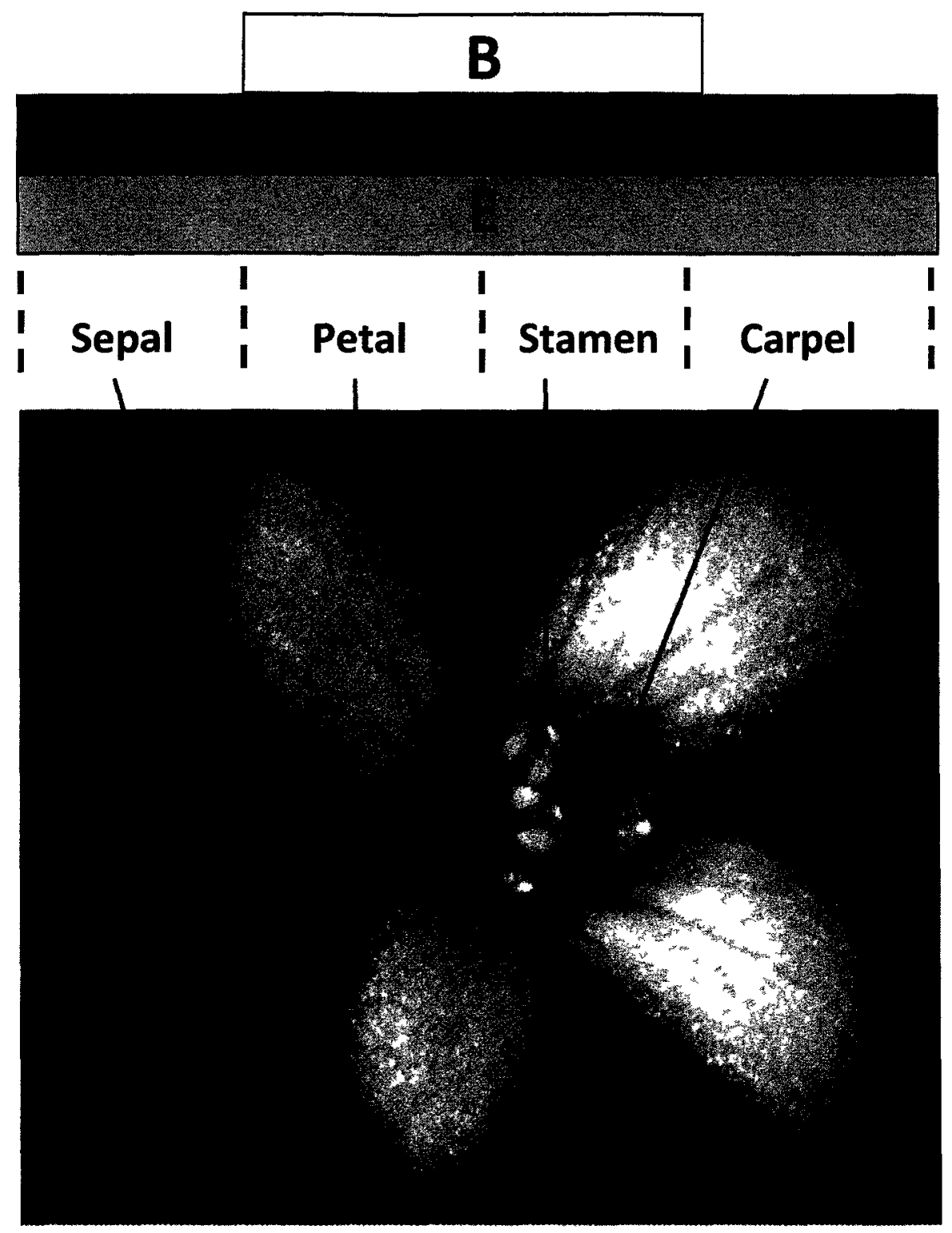

Figure 1.7 The ABCE model of floral development in Arabidopsis.

Floral-organ identity is defined by the overlapping expression domains of three sets of genes, termed Class A, B, and C that function in association with Class $\mathrm{E}$ genes that are expressed in all four whorls. Class A genes alone specify sepal identity; Class A+B genes specify petal identity; Class $\mathrm{B}+\mathrm{C}$ genes specify stamen identity; and Class $\mathrm{C}$ genes alone specify carpel identity. Class $\mathrm{E}$ genes are required in all four whorls of the flower. 


\section{Chapter 2}

\section{Arabidopsis BLADE-ON-PETIOLE1 and 2 promote floral meristem fate and determinacy in a previously undefined pathway targeting APETALA1 and AGAMOUS-LIKE24}

This chapter is presented essentially as published in Plant Journal (2010) 63, 974-989

M. Xu., T. Hu, S. McKim, J. Murmu, G.W. Haughn, and S.R. Hepworth

I carried out all of the experiments described in this Chapter with the following exceptions: Sarah McKim provided the scanning electron micrographs and BiFC data, Tieqiang Hu provided the qRT-PCR data for $A P 1, A G L 24, S O C 1$, and FUL transcripts, and Jhadeswar Murmu made the $p B O P 1: \because B O P 1-G F P$ plants used for ChIP assays. This article is reprinted with permission of the publisher (John Wiley and Sons). 


\begin{abstract}
The transition to flowering is a tightly controlled developmental decision in plants. In Arabidopsis, LEAFY $(L F Y)$ and APETALA1 (API) are key regulators of this transition and expression of these genes in primordia produced by the inflorescence meristem confers floral fate. Here, we examine the role of architectural regulators $B L A D E-O N-P E T I O L E 1(B O P 1)$ and $B O P 2$ in promotion of floral meristem identity. Loss-of-function bopl bop 2 mutants show subtle defects in inflorescence and floral architecture but in combination with lfy or apl, synergistic defects in floral meristem fate and determinacy are revealed. The most dramatic changes occur in bopl bop2 ap 1-1 triple mutants where flowers are converted into highly branched inflorescencelike shoots. Our data show that BOP1/2 function distinctly from LFY to upregulate $A P 1$ in floral primordia and that all three activities converge to down-regulate flowering-time regulators including $A G A M O U S-L I K E 24$ in stage 2 floral meristems. Subsequently, BOP1/2 promote Aclass floral-organ patterning in parallel with LFY and AP1. Genetic and biochemical evidence support the model that $\mathrm{BOP} 1 / 2$ are recruited to the promoter of $A P 1$ through direct interactions with TGA bZIP transcription factors, including PERIANTHIA. These data reveal an important supporting role for BOP $1 / 2$ in remodeling shoot architecture during the floral transition.
\end{abstract}




\subsection{Introduction}

The switch from vegetative to reproductive development in Arabidopsis is a tightly controlled process mediated by multiple genetic pathways in response to developmental cues and environmental signals (Kobayashi and Weigel, 2007; Turck et al., 2008). Inputs from floweringtime pathways converge to regulate the expression of a small number of genes with floral integrator activity including LEAFY (LFY), FLOWERING LOCUS T $(F T)$ and SUPPRESSOR OF OVEREXPRESSION OF CONSTANSI (SOC1) whose up-regulation in shoot apices promotes the production of an inflorescence (Parcy, 2005). The MADS-box flowering-time genes AGAMOUSLIKE24 (AGL24), SOC1, and SHORT VEGETATIVE PHASE (SVP) encode key determinants of inflorescence meristem identity in Arabidopsis (Hartmann et al., 2000; Michaels et al., 2003; Yu et al., 2004; Liu et al., 2007). Early in the transition to flowering, the inflorescence meristem produces cauline leaves, which generate secondary inflorescences in their axils. Subsequently, leaf development is fully repressed and lateral shoots acquire floral fate (Sablowski, 2007).

Two key regulators of floral meristem identity in Arabidopsis are LFY and APETALA1 $(A P 1)$. Expression of these genes in lateral organ primordia confers floral fate (Blázquez et al., 2006). The initial up-regulation of $L F Y$ specifies floral meristems by activating floral meristem identity genes including the MADS-box transcription factors encoded by $A P 1$ and CAULIFLOWER (CAL) and the homeodomain leucine-zipper (HD-ZIP) transcription factor encoded by LATE MERISTEM-IDENTITY1 (LMII) (Kempin et al., 1995; Liljegren et al., 1999; Saddic et al., 2006). LFY is a direct regulator of API (Parcy et al., 1998; Wagner et al., 1999) but activation is also directed by a complex of FT/FD (Abe et al., 2005; Wigge et al., 2005) and by SQUAMOSA PROMOTER BINDING PROTEIN-LIKE (SPL) proteins that control age related flowering time (Wang et al., 2009; Yamaguchi et al., 2009). 
Prior to their specification of floral organ identity, AP1 and LFY activities block the continued expression of inflorescence meristem identity genes in the floral meristem so that these shoots become determinate and develop as flowers (Yu et al., 2004; Liu et al., 2007; Liu et al., 2009a). Over-expression $A G L 24, S O C 1$, or $S V P$ partially transforms floral meristems into inflorescence meristems leading to branched flowers and floral bracts. These phenotypes are thereby suppressed in lfy and apl by loss-of-function mutations in agl24, socl, or $s v p$ (Yu et al., 2004; Liu et al., 2007). After commitment to flowering, activation of AGAMOUS (AG) in the dome of the floral meristem leads to repression of the stem-cell organizer WUSCHEL (WUS) ensuring that shoot determinacy is complete (Lenhard et al., 2001; Lohmann et al., 2001).

$B L A D E-O N-P E T I O L E 1(B O P 1)$ and BOP2 encode BTB-ankyrin transcriptional coregulators that are expressed in lateral organ boundaries and that control the architecture of leaves, fruits, and flowers. bop1 bop2 mutants develop leafy petioles and receptacle defects in flowers. Flowers have bracts and two petalloid structures typically replace the abaxial sepal (Hepworth et al., 2005; Norberg et al., 2005; McKim et al., 2008). The role of BOP1/2 is best understood in leaves where they are direct activators of the lateral organ boundary marker ASYMMETRIC LEAVES2 (Jun et al., 2010). Co-misexpression of meristematic genes such as BREVIPEDICELLUS $(B P)$ and the blade-promoting transcription factor encoding $J A G G E D$ $(J A G)$ in bop1 bop2 mutants promotes indeterminacy in leaf petioles leading to ectopic leaflet formation (Ha et al., 2003, 2004, 2007; Norberg et al., 2005).

Two previous reports have provided preliminary evidence that the $B O P$ genes also promote floral meristem identity. In situ experiments by Karim et al. (2009) have suggested that the redundant activities of BOP1/2 and PUCHI, an EREBP transcription factor, promote $L F Y$ expression in lateral meristems. In bop1 bop2 puchi triple mutants, extra inflorescence like 
shoots arise with the eventual production of bopl bop2-like flowers. Norberg et al. (2005) also showed that bopl bop 2 enhances $1 f y-26$ in bract formation and floral meristem identity defects but the mechanism was not addressed.

Here, we use a genetics approach to examine the role of BOP1/2 in the floral transition. Loss-of-function bopl bop 2 mutants show minor defects in inflorescence and floral architecture but in combination with lfy or apl, synergistic defects in floral fate and shoot architecture are revealed. We show that BOP1/2 function in parallel with LFY to control determinacy in floral shoots through activation of $A P 1$ and repression of $A G L 24$ in developing flowers. To establish mechanism, we show that $\mathrm{BOP} 1 / 2$ are recruited to the promoter of $A P l$ in part through direct interactions with the TGA bZIP factor PERIANTHIA (PAN). These data reveal an important supporting role for BOP1/2 in remodeling shoot architecture during the floral transition.

\subsection{Materials and methods}

\subsubsection{Plant material and growth conditions}

Plants were grown on agar plates or in soil at $21^{\circ} \mathrm{C}$ in long-day $(16 \mathrm{~h})$ or continuous $(24 \mathrm{~h})$ light. Wild-type was the Columbia-0 (Col-0) ecotype of Arabidopsis thaliana. Mutant alleles were obtained from the Arabidopsis Biological Resource Center unless otherwise stated. bop1-3 bop21 and pan-1 mutants were previously described (Hepworth et al., 2005). The ap1-1 allele was introgressed into Col-0. The agl24-3 allele (SALK_095007) was provided by Richard Amasino and $f t-1$ (introgressed into Col-0), $f d-2$, and $f d-2$ lfy-12 were gifts from Hao Yu and Philip Wigge. Mitsuhiro Aida provided the bopI bop2 puchi mutant and Detlef Weigel provided spl9 and spl9 pSPL9:GFPSPL9 lines. All mutant combinations were constructed by crossing and confirmed by genotyping. For genotyping lfy-2, a 168-bp product was amplified by PCR; BamHI cleaves only the wild-type product. For genotyping $f t-1$, a 183-bp product was amplified by PCR; SacII 
cleaves only the wild-type product. Floral stages were determined according to Smyth et al. (1990). Primers are listed in Table 2.1.

\subsubsection{Scanning electron microscopy (SEM)}

Samples were prepared for SEM as described (Hepworth et al., 2005). Images were acquired on Hitachi VP-6400 (http://www.hitachi-hitec.com) or Tescan Vegall XMU VPSEM (http://www.tescan-usa.com) microscopes.

\subsubsection{BOP2::GUS reporter lines, GUS staining and in situ hybridization}

pBOP 2::GUS containing the BOP2 5'-UTR (nt -4015 to +16 ) fused in-frame with the GUS gene uidA was created using the strategy described (Hepworth et al., 2002). Wild-type plants were transformed by floral dipping (Clough and Bent, 1998). Tissues were stained for GUS activity, fixed, embedded, and sectioned as described in Sieburth and Meyerowitz (1997). Sections were adhered to glass slides and de-waxed with tert-butanol prior to imaging. In situ hybridization and probe synthesis was performed essentially as described (Hepworth et al., 2005). The $A G$ probe was based on Drews et al. (1991).

\subsubsection{RT-PCR and quantitative RT-PCR (qPCR)}

Total RNA was isolated from inflorescence apices using Trizol reagent (Invitrogen, http://www.invitrogen.com). Total cDNA was synthesized from $1 \mu \mathrm{g}$ of RNA template using Superscript III reverse transcriptase (Invitrogen) followed by RT-PCR using Taq polymerase (Invitrogen). GAPC served as a control transcript (Hepworth et al., 2005). Band intensities were quantified with AlphaImager software (Cell Biosciences, http://www.cellbiosciences.com). qPCR was performed in triplicate with SYBR Green (Sigma, http:// www.sigmaaldrich.com) and IQ Supermix (BioRad, http://www.bio-rad.com) using a Rotor-Gene 6000 (Qiagen, 
http://www.qiagen.com) thermocycler. Conditions were optimized for each primer pair and data quality was verified by melting curve analysis. qPCR was performed with triplicates and relative transcript levels were calculated from mean of threshold cycle values and standard curves. Values were normalized to $G A P C$ and then to the wild-type control. Experiments were repeated twice with independently-isolated RNA to ensure reproducibility.

\subsubsection{ChIP assay}

$1 \mathrm{~g}$ of 25-day-old bopl bop2 BOP1::BOP1-GFP apices were used for ChIP as described in Saleh et al. (2008) omitting the nuclear isolation step. Anti-GFP antibodies were used for immunoprecipitation (ab290; Abcam, http://www.abcam.com). Fold-enrichment of DNA sequences was determined by qPCR in triplicate with $A C T I N 2 / 7$ as a control according to Liu $e t$ al. (2008). Primers are listed in Table 2.2. Assays were repeated twice to ensure reproducibility.

\subsubsection{Yeast 2-hybrid analysis}

We used the GAL4-based yeast 2-hybrid system described in Kohalmi et al. (1998). For all pairwise interactions, the bait was BOP2 coding region fused in-frame with the GAL4 DNA-binding domain and the preys were PAN, FD, LFY, AGL24, SPL3, SPL9, SEP1, SEP2, SEP3, or SEP4 coding regions fused in-frame to GAL4 transcriptional activation domain. The BOP2 bait and PAN prey constructs were as described previously (Hepworth et al., 2005). All other prey constructs were constructed likewise. The coding sequences of $F D, L F Y, A G L 24, S P L 3, S P L 9$, SEP1, SEP2, SEP3, and SEP4 were amplified by PCR using cDNA derived from inflorescences apices as the template and iProof as the polymerase (Biorad, www.bio-rad.com). PCR products were subcloned into pCR-BluntII-TOPO (Invitrogen, www.invitrogen.com) and sequenced to ensure fidelity. Recognition sites for restriction enzymes were incorporated at the 5 ' ends of the primers used for amplification to facilitate their subsequent directional cloning into the prey 
plasmid pBI-881 (Kohalmi et al., 1998). Co-transformation of yeast with bait and prey plasmids and quantitative determination of $\beta$-galactosidase activity were as described previously (Hepworth et al., 2005).

\subsubsection{Bimolecular fluorescence complementation (BiFC)}

Constructs were based on the pSAT modular vectors modified for BiFC, gifts from Dr. Stanton Gelvin (Citovsky et al. 2006). The pSAT6A vector contained full-length EYFP (Invitrogen, www.invitrogen.com), pSAT1A contained the C-terminal half of EYFP (residues 1-174) and pSAT4A contained the N-terminal end of EYFP (residues 175-stop). The coding sequences of $B O P 2$ and $P A N$ were amplified by PCR from cloned cDNA template using primers incorporating $K p n 1$ sites at the 5 ' end. Products were digested with $K p n I$ and ligated into the corresponding site of each pSAT vector. All constructs were sequenced to confirm fidelity and translational fusion. Protoplasts were transformed according to Yoo et al. (2007) using $20 \mu \mathrm{g}$ of plasmid DNA for single vector controls and $10 \mu \mathrm{g}$ of each plasmid for bimolecular fluorescent complementation co-transformation. Protoplasts were allowed to rest for 12-20 hours before visualization by epifluorescent microscopy.

\subsection{Results}

\subsubsection{Dynamic pattern of $B O P$ expression in the inflorescence apex}

To clarify the dynamics of $B O P$ expression during floral development, we examined the expression pattern of a GUS reporter gene driven by the $B O P 2$ promoter. $B O P 2$ expression quickly cleared from lateral inflorescence meristems and localized to the axil of cauline leaves (Figure 2.1a). Expression was detected in floral anlagen (stage 0 ) and in the adaxial portion of stage 1 floral primordia (Figure 2.1c, d). At late stage 2, expression shifted to the boundary 
between the floral primordium and the cryptic bract (Figure 2.1a, b, d, e, asterisks). BOP2 expression was focused in the dome of the floral meristem at early stage 3 but shifted to the sepal axils and was maintained (Figure 2.1c, $\mathrm{f}, \mathrm{g}$, arrows). BOP $:: G U S$ was expressed in a similar pattern (not shown). These data are in agreement with previous in situ data (Hepworth et al., 2005; Norberg et al., 2005; Karim et al., 2009) and are consistent with a role for BOP1/2 in promotion of floral meristem identity.

\subsubsection{Weak floral meristem identity defects in bop1 bop2 mutants}

Close inspection of bop1 bop2 plants grown in inductive photoperiods revealed phenotypes consistent with mild defects in floral meristem identity. Some of these defects were reported previously but here they are considered collectively. First, visible bracts subtended about $20 \%$ of flowers grown in continuous light (Table 2.3 and Figure 2.2a). Hepworth et al. (2005) showed that bracts are initiated on most flowers at stage 1-2 but that their further development is variable. Second, bopl bop 2 mutants displayed a small but reproducible increase in secondary inflorescences compared with wild-type (Figure 2.2b; Norberg et al., 2005). Cauline leaves were sometimes absent from the base of shoots preceding the node of first flower (Figure 2.2c). Sometimes this was due to ectopic pedicel elongation causing displacement of the cauline leaf but other times the cauline leaf was lacking indicating that its development was repressed. Third, branched flowers arose at a low frequency (Figure 2.2d; Table 2.3; Ha et al., 2007). This phenotype is characteristic of apl mutants, caused by the ectopic initiation of floral meristems in the axils of sepal-whorl organs, but occurs less commonly in lfy mutants (Irish and Sussex, 1990; Schultz and Haughn, 1991, 1993; Weigel et al., 1992). Overall, the range of floral meristem identity defects in bop1 bop 2 closely resembles those in lfy mutants consistent with the notion that like LFY, BOP1/2 promote floral fate. 


\subsubsection{Interactions with LEAFY and LATE-MERISTEM-IDENTITY1}

LFY is the central floral meristem identity regulator in Arabidopsis and its loss-of-function generates a large increase in secondary inflorescences, floral bracts, and some branched flowers (Schultz and Haughn, 1991; Weigel et al., 1992). Enlarged floral bracts and nodes without flowers were reported for bop 1 bop 2 crossed to the strong lfy-26 mutant suggesting that BOP1/2 and LFY contribute independently to floral meristem identity (Norberg et al., 2005). To examine this further, we crossed bopl bop2 mutants to strong (lfy-l) and weak (lfy-2) alleles and assayed the triple mutants for defects in floral meristem identity (Tables 2.3 and 2.5; Figure 2.3). In continuous light, $l f y-2$ plants generated about 3.8 more secondary inflorescences than wild-type but no further increase occurred in triple mutants with bop1 bop2 suggesting that BOP1/2 do not function redundantly with LFY to control the number of secondary inflorescences (Table 2.3). Comparison of lfy-2 mutants to bop1 bop2 lfy-2 triple mutants showed a significant increase in plants with branched flowers (54.4\% versus $100 \%)$ and floral bracts (4.2\% versus $100 \%)$. Branching patterns in bopl bop2 lfy-2 flowers were more complex than in $l f y-2$ with enhanced internode elongation between successive floral organs (Figure 2.3a-c). Bracts in bopl bop2 lfy-2 were enlarged and late in the primary inflorescence, nodes containing a bract but no flower developed (Figure $2.3 \mathrm{~d}, \mathrm{~g}$ ). Bracts and floral branching were also elaborated in bop1 bop 2 lfy-1 mutants (Figure 2.3e, f) confirming separate roles for BOP1/2 and LFY in promotion or maintenance of floral meristem identity.

LFY promotes flowering by activating a suite of downstream floral meristem identity regulators. $L M I 1$, which encodes an HD-ZIP transcription factor, is upregulated by LFY in stage 1 flowers before localizing to the cryptic bract (Saddic et al., 2006). Loss-of-function lmil enhances the number of secondary inflorescences in weak lfy mutants, showing that LMI1 
functions in part as a meristem-identity factor downstream of LFY. LMI1 has a second LFYindependent role in leaf and bract repression: in short-day photoperiods lmil mutants develop petiole leaflets similar to bopl bop2 mutants (Hepworth et al., 2005; Saddic et al., 2006). No increase in the number of secondary inflorescences or floral branching occurred in bop1 bop 2 Imil triple mutants (Table 2.3) nor were petiole leaflets in bop1 bop2 mutants enhanced (data not shown). However, the frequency of flowers with bracts was much higher in the triple mutant relative to bop1 bop2 (53.7\% versus 11.2\%; Figure 2.3o-r) indicating that BOP1/2 and LMI1 contribute separately to bract repression and have little impact on the number of secondary inflorescences when LFY is functional.

\subsubsection{Interactions with APETALA1}

The primary target of LFY in promotion of floral fate is $A P I$. Activation of $A P I$ together with $C A L$ and $L M I l$ feed-forward to reinforce $L F Y$ expression so that floral induction is sharp and unidirectional (Saddic et al., 2006). The strong apl-1 (Col) mutant shows elaborate floral branching only in the first few nodes after the switch to flowering (Table 2.3; Irish and Sussex, 1990; Schultz and Haughn, 1993). In bop1 bop2 ap1-1 triple mutants, floral meristems were dramatically converted to partial inflorescences, with curd-like apices similar to ap 1 cal mutants (Figure 2.4a-e; Bowman et al., 1993). Highly branched shoots, often indeterminate, developed in place of all floral nodes. Single peduncles showed enhanced floral branching and internode elongation between successive floral organs (Figure $2.4 \mathrm{f}-\mathrm{j}$; Tables 2.3 and 2.5). Floral bracts in stage 1-2 flowers of the triple mutant were highly developed relative to parental controls and first whorl organs showed spiral instead of whorled phyllotaxy (Figure 2.5). Branching was enhanced in bop1 bop2 ap1-12 mutants but all shoots remained determinate, likely due to residual AP1 function in ap1-12 (Table 2.3 and Figure 2.41-m). These interactions reveal that 
BOP1/2 and AP1 redundantly suppress inflorescence characteristics in floral shoots.

\subsubsection{BOP activity has A-class function in flowers}

After flowers are initiated, LFY and AP1 promote floral patterning. Floral organ identity is determined by the overlapping activities of three classes of homeotic genes termed A, B, and C that specify sepals, petals, stamens and carpels according to the $\mathrm{ABC}$ model (Haughn and Somerville, 1988; Coen and Meyerowitz, 1991). Strong lfy mutants lack petals and stamens due lack of B-class gene activation and sepal whorl organs become carpelloid as a result of $A G$ misexpression (Drews et al., 1991; Weigel and Meyerowitz, 1993; Liu and Meyerowitz, 1995). Comparison of lfy-2 and bopl bop2 lfy-2 triple mutants showed enhancement of lfy floral organ identity defects (Table 2.4 and Figure $2.3 \mathrm{c}, \mathrm{j}-\mathrm{m}$ ). Whereas weak $l f y-2$ mutants develop some petals and stamens, sepal-like or mosaic sepal/carpel organs formed in all whorls of bopl bop2

lfy-2 flowers, with $A G$ misexpression in the outer whorls (Figure 2.3i). Mutation of bopl bop2 similarly enhanced the carpelloid character of outer-whorl organs in strong $l f y-1$ mutants (Table 2.4 and Figure 2.3h, n) similar to lfy apl mutants (Schultz and Haughn, 1993). AP1 also contributes to A-function by specifying sepal and petal identity (Irish and Sussex, 1990; Schultz and Haughn, 1991; Mandel et al., 1992). Flowers in bopl bop2 ap1-12 triple mutants closely resembled those in the strong apl-1 mutant, lacking petals (Figure 2.4g, 1, m; Table 2.4). In bop1 bop2 ap 1-1 triple mutants, sepal-whorl organs gained carpelloid features (Figure 2.4k and Table 2.4) consistent with misexpression of $A G$ in sepal margins (data not shown). Overall, these data reveal that $\mathrm{BOP} 1 / 2$ promote A-class floral patterning.

\subsubsection{BOP1/2 and LFY are distinct regulators of $A P 1$ in floral meristems}

Given that BOP activity promotes floral meristem identity, we reasoned that $L F Y$ and/or $A P 1$ might be targets of regulation. In situ hybridization by Karim et al. (2009) showed a dramatic 
lack of $L F Y$ expression in bop1 bop2 puchi apices, providing one explanation for the severe flower-to-shoot reversions observed in bop1 bop 2 apl-1 triple mutants. We therefore monitored $L F Y$ expression in the inflorescence apices of wild-type, bop1 bop2, ap1-1, and bop1 bop2 ap1-1 plants grown in continuous light, using bop1 bop 2 puchi triple mutants as a control. Apices were examined at three time-points: 14-day-old seedlings committed to flowering, 1-cm bolts, and 5$\mathrm{cm}$ bolts. However, $L F Y$ transcript accumulation in both triple mutants was similar to ap1-1 control apices in which $L F Y$ accumulates to WT levels (Figure 2.6; Weigel et al., 1992) indicating that the dramatic flower to shoot phenotypes in bop1 bop2 ap1-1 mutants cannot easily be attributed to lack of $L F Y$ expression.

Rather, in situ hybridization revealed that in combination with all mutant genotypes examined, loss of BOP activity had a significant impact on $A P 1$ transcript levels. $A P I$ is independently activated by LFY and FD/FT in stage 1 flowers and represents the earliest known marker of commitment to floral fate (Hempel et al., 1997; Wagner et al., 1999; Abe et al., 2005; Wigge et al., 2005). As seen previously, $A P l$ transcript in $l f y-1$ and $l f y-2$ apices was reduced (Figure 2.7; Mandel and Yanofsky, 1995a; Ruiz-Garcia et al., 1997; Liljegren et al., 1999). In lfy- 1 apices, API first accumulated in the sepal whorl of stage 3 flowers and slightly earlier in lfy-2 apices (Figure $2.7 \mathrm{~g}-\mathrm{i}, \mathrm{m}-\mathrm{o}$ ). In contrast, little or no $A P 1$ accumulated in bop 1 bop 2 lfy- 1 or bop1 bop 2 lfy-2 flowers at stage 1-2, nor was there expression at stage 3 in bopl bop 2 lfy-1 flowers (Figure 2.7j-1, p-r). Reduced $A P I$ expression was also apparent in bop1 bop2 puchi control apices (Figure $2.7 \mathrm{~s}-\mathrm{j}$ ). These data identify $A P I$ as a major target of BOP regulation.

To examine this further, we used qPCR to monitor the expression of $L F Y$ direct targets $A P I$ and $C A L$ in the inflorescence apices of lfy-1 versus bopl bop 2 lfy- 1 triple mutants with $5-\mathrm{cm}$ bolts. Both genes are expressed specifically in floral primordia (Wagner et al., 1999; Ferrandiz et 
al., 2000; William et al., 2004). A dramatic reduction in AP1 transcript was observed in bop1 bop2 lfy-1 triple mutants; $C A L$ levels were also slightly reduced (Figure 2.8a). We further monitored $A P 1, C A L$, and $L M I 1$ transcript levels: (i) in apices of lfy-l versus bopl bop2 lfy-1; and (ii) in $f d-2$ versus $f d-2$ lfy-12 control apices; using the experimental design of Abe et al. (2005) (1-cm bolts; Figure 2.8b). Dramatically lower levels of $A P 1$ were observed in bop1 bop2 lfy- 1 mutants relative to $l f y-1$ mutants [comparable with $f d-2$ versus $f d-2$ lfy-12 apices (Abe et al., 2005; Wigge et al., 2005)] and in bopl bop2 puchi control apices. CAL and LMII transcripts were also slightly reduced in bopl bop 2 lfy-1 triple mutants compared with lfy-1, suggesting that BOP1/2 also have some effect on genes other than AP1. This finding promoted us to test if BOP activity resides in the FT pathway. However, levels of $A P 1$ and $C A L$ in bopl bop $2 f t-1$ apices were much lower than in $f t-1$ single mutants (Figure $2.8 \mathrm{~b}$ ) and bolting was greatly delayed in bopl bop $2 \mathrm{ft}-1$ triple mutants (apex first visible at $54.2 \pm 0.66$ days, $\mathrm{n}=33$ ) compared with $f t-1$ mutants $(44.4 \pm 0.55$ days, $\mathrm{n}=34)$ and $f t-10$ null mutants $(47.8 \pm 0.55$ days; $\mathrm{n}=24)$. These genetic data indicate that BOP1/2 promote floral meristem identity independently of LFY and FT/FD.

\subsubsection{BOP activity contributes to down-regulation of inflorescence identity genes}

During the transition to flowering, $A G L 24, S O C 1$, and $F U L$ are up-regulated in shoot apices to drive the production of primary and secondary inflorescences (Mandel and Yanofsky, 1995; Hempel et al., 1997; Ferrandiz et al., 2000; Yu et al., 2002; Michaels et al., 2003). Together with AP1, these factors initially promote floral meristem fate but are subsequently down-regulated to permit development of the determinate floral shoot (Yu et al., 2004; Liu et al., 2007, 2009b; Gregis et al., 2008). The architecture of floral nodes in bop1 bop 2 apl and bopl bop 2 lfy-2 triple mutants suggests that BOP activity contributes to this down-regulation. Supporting this, qPCR 
analysis showed elevated $A G L 24, S O C 1$, and $F U L$ transcript in triple mutant apices relative to control apices (Figure 2.8c). In situ hybridization was used to monitor the expression patterns of these genes in more detail.

In wild-type and bopl bop2 apices, $A G L 24$ was expressed in the inflorescence meristem and floral primordia until late stage 2 when expression became restricted to the cryptic bract (Figure 2.9a, b; Michaels et al., 2003). At stage 3, when floral organs begin to differentiate, AGL24 expression sometimes occurred in the dome of bop1 bop2 flowers (Figure 2.9d). In ap11 and $l f y-2$ control apices, ectopic expression of $A G L 24$ was consistently detected in the dome and/or sepal whorl of stage 2 and 3 flowers (Figure 2.9f, g, h, i). Misexpression was dramatically enhanced in bopl bop2 ap1-1 and bopl bop2 lfy-2 triple mutants, detected throughout stage 1-3 floral primordia, consistent with their partial conversion into inflorescences (Figure 2.9j, k). Misexpression of $S O C 1$ and FUL in triple mutant apices was similar to AGL24 (Figures 2.10 and 2.11). These data indicate that BOP $1 / 2, \mathrm{AP} 1$, and LFY activities converge at stage 2 to downregulate genes that confer inflorescence identity.

\subsubsection{Rescue of floral branching but not bract formation by loss-of-function agl24}

Loss-of-function agl24 rescues floral branching in ap 1-1 and bract formation in lfy-6 (Yu et al., 2004; Liu et al., 2007; Ler ecotype) identifying misexpression of $A G L 24$ as a leading cause flower-to-shoot reversion in these mutants. Whilst loss-of-function agl24 did not significantly reduce floral branching in bopl bop2, lfy-2, or ap 1-1 plants (Col ecotype), branching in the triple mutants bopl bop2 ap1-1 and bop1 bop 2 lfy-2 was dramatically rescued as evidenced by steep reductions in the average number of flowers per peduncle and reestablishment of determinacy at all floral nodes in bop1 bop2 ap1-1 plants (Table 2.5; Figures 2.12 and 2.13). These data confirm that shoot architectural defects in bopl bop 2 lfy-2 and bopl bop2 ap1-1 triple mutants are due in 
part to continued expression of $A G L 24$ in floral meristems.

\subsubsection{Evidence that $\mathrm{BOP} 1 / 2$ are recruited to the $A P 1$ promoter by direct interaction with PAN}

BOP1/2 are transcriptional regulators of $A P 1$ and $A G L 24$ in floral meristems raising the possibility that this regulation is direct. $\mathrm{BOP} 1 / 2$ are $\mathrm{BTB}$-ankyrin proteins similar to the pathogen defense regulator NPR1, a transcriptional co-activator that exerts most or all of its function via TGA bZIP transcription factors (Zhang et al., 1999; Despres et al., 2000; Rochon et al., 2006; Boyle et al., 2009). BOP similarly interacts with a subset of TGA factors including PAN and BOP1/2-PAN function in the same genetic pathway to control perianth floral organ number (Hepworth et al., 2005). Several other TGA factors are broadly expressed in inflorescence apices (e.g. Li et al., 2009; Maier et al., 2009) suggesting that BOP1/2 may function through one or more of these factors to promote floral meristem identity. Supporting this, pan-1 mutants display floral meristem identity defects similar to bop 1 bop 2 mutants, albeit at a lower frequency: floral bracts $(2.8 \%$ of plants, $n=71)$, cauline leaves absent from the base of shoots preceding node of first flower ( $5.6 \%$ of plants, $n=71$ ), and branched flowers ( $2.68 \%$ of node 1-5 flowers, $\mathrm{n}=149$ ) (Figure 2.14a-c). Using bimolecular fluorescence complementation (BiFC) assays, BOP-PAN interaction was confirmed in the nucleus of Arabidopsis mesophyll cells (Figure 2.15b-j). Conversely, no interaction in yeast was detected between BOP proteins paired with LFY, FD, AGL24, SPL3, SPL9, or SEPALATTA1-4 (Figure 2.15a; data not shown) representing other direct regulators of $A P 1$ and $A G L 24$ (Wagner et al., 1999; Wigge et al., 2005; Liu et al., 2007, 2008; Gregis et al., 2008; Wang et al., 2009; Yamaguchi et al., 2009). Using the AthaMap tool (Galuschka et al., 2007) we identified potential TGA binding sites in the promoter of AP1 (Figure 2.14d) and tested for BOP1 occupancy at these sites using chromatin 
immunoprecipitation (ChIP) assays. These assays used bop1 bop 2 plants complemented by a pBOP1:BOP1-GFP fusion protein. Strong and selective occupancy of BOP1-GFP was reproducibly detected at sites 1 and 3 in the $A P 1$ promoter (Figure 2.14f). Site 3 maps close to binding sites for FD, LFY, and SPL3/9 in the main control region for APl (Parcy et al., 1998; Wigge et al., 2005; Wang et al., 2009; Yamaguchi et al., 2009). Enrichment of GFP-SPL9 at site 1 in the AP1 promoter served as a positive control (Figure 2.14f; Wang et al., 2009). These results collectively provide strong evidence that $\mathrm{BOP} 1 / 2$ are recruited to sites in the $A P 1$ promoter via TGA binding factors where they function as transcriptional co-regulators thereby expanding the role of TGA transcription factors in development and revealing a previously undefined pathway for promotion of floral fate.

\subsection{Discussion}

In this study, we use a genetics approach to examine the role of architectural regulators BOP1 and BOP2 in promotion of floral fate. These genes are expressed in lateral organ primordia, including floral meristems, together with regulators of floral meristem identity including LFY, LMI1, and AP1. Loss-of-function bop1 bop 2 show only subtle defects in floral fate making their function more easily analyzed in sensitized genetic backgrounds. Double mutants with $l f y, a p l$, and Imil mutants define in detail how BOP activity promotes floral meristem identity (Figure 2.16). We show that BOP $1 / 2$ promote flowering distinctly from LFY, contributing to activation of $A P 1$ and repression of inflorescence identity genes including $A G L 24, S O C 1$, and $F U L$, crucial for generating a determinate floral shoot. At stage 3, BOP1/2 exerts A-class floral patterning activity in parallel with LFY and AP1. Several lines of evidence support the model that BOP1/2 bind in vivo to regulatory sequences in the $A P 1$ promoter through direct interaction with TGA transcription factors, including PAN. 


\subsubsection{BOP activity is required for maintaining floral fate}

Mutations that impair floral meristem identity cause the full or partial reversion of flowers into lateral branches. Delayed commitment to floral fate is associated with extra secondary inflorescences and floral bract outgrowth corresponding to defects in LFY expression or activity (Liu et al., 2009a). In ap 1-1 mutants, $L F Y$ transcript accumulation is normal but maintenance of floral fate is compromised leading to branched flowers (Weigel et al., 1992). The combination of bopl bop 2 with apI-1 also shows robust expression of $L F Y$ throughout the floral transition and normal levels of $C A L$ (data not shown) but extreme floral branching and indeterminate shoot growth, indicating that $\mathrm{BOP} 1 / 2$ plays a strong role in commitment to floral fate. The situation is similar in bop1 bop2 puchi plants (see also Karim et al., 2009). Moreover, bop1 bop2 mutations fail to increase the formation of secondary inflorescences in weak lfy and lmil mutants but rather increase bract formation and floral branching. These data indicate that BOP1/2 function in parallel with LFY and that the redundant activities of BOP $1 / 2$ and AP1 enforce floral fate.

\subsubsection{Down-regulation of flowering-time genes}

In stage 2 floral meristems, down-regulation of inflorescence identity genes prevents continuation of the shoot developmental program and permits LFY and AP1 to initiate differentiation of floral organs (Liu et al., 2009a). AGL24, SOC1, and SVP are directly repressed by AP1, whereas repression by LFY is indirect (Yu et al., 2004; Liu et al., 2007; Gregis et al., 2008). BOP $1 / 2$ contributes to this down-regulation as evidenced by dramatic misexpression of $A G L 24, F U L$, and SOC1 in bop1 bop2 ap1-1 and bop1 bop 2 lfy-2 apices. SVP was not a target of BOP repression (data not shown). Loss-of-function agl24 strongly suppressed floral branching in triple mutants but rescue was incomplete, likely due to continued misexpression of $S O C l$ and FUL. It remains unclear if BOP $1 / 2$ directly repress $A G L 24$, but the promoter contains two TGA 
binding motifs (not shown). In petioles, BOP1/2 promotes determinacy by repression of $B P(\mathrm{Ha}$ et al., 2003, 2007; Chapter 3) but the mechanism appears to differ in flowers as $B P$ is not misexpressed in triple mutant apices nor do $b p$ mutations rescue floral branching (data not shown).

\subsubsection{Bract formation}

Arabidopsis flowers develop in the absence of a visible bract, which is specified but repressed in its development (Hepworth et al., 2006). The number and/or size of bracts was enhanced in all double mutant combinations tested: lfy lmil, bopl bop2 lfy, bop1 bop2 lmil, bopl bop2 apl, and bopl bop 2 agl24-3, indicating that several inter-related pathways contribute to bract repression. Of these, $L M I I$ and $A G L 24$ expression localizes to the cryptic bract in late stage 2 whereas $B O P 1 / 2$ expression localizes to the boundary between the floral meristem and the cryptic bract (this study; Karim et al., 2009; Saddic et al., 2006). LFY and API are expressed in the floral meristem contributing non-cell autonomously to bract repression (Sessions et al., 2000; Hepworth et al., 2006). One mutual target of repression is $J A G$, whose mutation alleviates bract formation in lfy and strong apl mutants (Dinneny et al., 2004; Ohno et al., 2004). BOP1/2 also represses $J A G$ in the cryptic bract but jag mutations fail to rescue bract formation in bopl bop2 mutants supporting the involvement of additional factors (Norberg et al., 2005).

\subsubsection{BOP1/2 and LFY are independent regulators of $A P 1$}

BOP1/2 and LFY are co-expressed in floral anlagen and activate $A P 1$, a key marker of commitment to floral fate. Apical expression of $A P I$ is strongly delayed in lfy mutants and essentially abolished in $l f y f t$ and $l f y f d$ double mutants indicating that FT/FD and LFY are the major direct regulators of API expression (Parcy et al., 1998; Abe et al., 2005; Wigge et al., 2005). SPL transcription factors provide additional positive input (Wang et al., 2009; Yamaguchi 
et al., 2009). In lfy ft double mutants, leaves are generated in place of flowers (e.g. Ruiz-Garcia et al., 1997). Similar nodes containing only a bract occurred late in the primary inflorescence of bop1 bop2 lfy plants providing evidence that BOP and LFY are independent regulators of floral meristem fate. ChIP analysis showed enrichment of BOP1-GFP at two potential TGA binding sites in the AP1 promoter close to binding sites for LFY, FD, and SPL3/9 (Parcy et al., 1998; Wigge et al., 2005; Wang et al., 2009; Yamaguchi et al., 2009). Consistent with this, we found weak floral meristem identity defects in pan-1 mutants similar to bopl bop 2 and show that BOP2 interacts selectively with PAN over known direct regulators of AP1 expression. BOP1/2 interact in yeast with several TGAs, including PAN, which functions in the same genetic pathway as BOP1/2 to control sepal number in flowers (Hepworth et al., 2005). Several other TGAs are expressed in inflorescence apices including TGA2, TGA3, and TGA7, which interact with the floral glutaredoxin ROXY1 (Xing et al., 2005; Li et al., 2009) and TGA4, which binds in vitro to the FT promoter and with CONSTANS, a direct regulator of FT (Samach et al., 2000; Song et al., 2008). One of these may preferentially contribute to repression of $A G L 24$ since no enhancement of floral branching occurs in pan-1 apl-1 mutants (data not shown). Thus far, genetic redundancy has hampered our attempts to identify which of these might function with PAN in the floral transition.

\subsubsection{BOP activity regulates shoot architecture by controlling determinacy}

The transition to flowering involves dramatic changes in shoot architecture, beginning with the formation of primary and secondary inflorescences and ending with the production of flowers. How changes in shoot architecture are coordinated with the decision to flower remains unclear. In leaves, BOP $1 / 2$ control the determinacy and hence architecture of petioles through corepression of $K N O X I$ homeobox genes and blade-growth regulators such as $J A G$ (Ha et al., 2004, 
2007; Jun et al., 2010). This study shows that BOP1/2 likewise control determinacy and architecture of floral shoots through activation of $A P 1$ and repression of $A G L 24$. Shoot determinacy further depends on the activation of $A G$ at the dome of the floral meristem causing termination of the stem cell population. LFY and WUS are the main activators of $A G$ (Busch $e t$ al., 1999; Lenhard et al., 2001; Lohmann et al., 2001) but positive input is also provided by PAN (Das et al., 2009; Maier et al., 2009). Similar to pan-2 lfy mutants, unfused carpels occur in bopl bop2 lfy-1 flowers (Das et al., 2009; Figure 2.3h) indicating that BOP1/2 may likewise fine-tune $A G$ expression in developing flowers, but this remains to be tested. Unlike flowering-time regulators, BOP1/2 are broadly expressed in initiating lateral organs and their loss-of-function affects leaf, fruit, and floral architecture (e.g. Ha et al., 2003; Hepworth et al., 2005; McKim et al., 2008). Plants over-expressing BOP1 or 2 are reduced in stature with clustered or downwardpointing siliques, similar to mutation of BP or PENNYWISE (PNY) (Norberg et al., 2005; Ha et al., 2007). Interestingly, double mutants of pny and the related gene poundfoolish (pnf) cannot complete floral evocation: flowering signals direct the up-regulation of floral integrators $F T$, $S O C 1$, and $F U L$ but $L F Y$ and $A P I$ are not activated nor is an inflorescence generated (Smith $e t$ al., 2004; Kanrar et al., 2008) indicating that PNY/PNF coordinate shoot architecture and flowering. Our work indicates that BOP1/2 are likely also involved in this co-ordination and illustrates how modulation of determinacy contributes to shoot architectural diversity. 
Table 2.1 Primers used for genotyping, making BOP2::GUS construct, $\mathrm{qPCR}$ analysis, and making in situ hybridization probes.

\begin{tabular}{|c|c|}
\hline Primer & Sequence 5'-3' \\
\hline \multicolumn{2}{|l|}{ Genotyping } \\
\hline Ify-2 dCAPs $F$ & GTTTGGGGACAGAGAGACAGAGGGAGGATC \\
\hline Ify-2 dCAPs R & CGCCACGGTCTTTAGCAATTGTCTGG \\
\hline agl24-3 F & GAATGAGAGACATATTGGGAAGGTA \\
\hline agl24-3 R & AAGTGTCGGAGTCATCCTCAAG \\
\hline 4H Salk RP & CGTACCCTTTGATTTTAGTATGCTG \\
\hline 4H Salk LP & GCACAATCTTTCGACTTCATCACC \\
\hline 5H Salk RP & CCCTTTTTATAATCAGCATCAAGA \\
\hline 5 H Salk LP & TCGACGCCGAAGTAACGAGAG \\
\hline $\mathrm{ft}-1-\mathrm{F}$ & TACAATTGTCAGAGGGAGAGTGGCCGCG \\
\hline $\mathrm{ft}-1-\mathrm{R}$ & CACTTATATATTGAACTACTATAGGCATCAT \\
\hline \multicolumn{2}{|l|}{ GUS reporter } \\
\hline BOP2-4kb-Bam-F1 & ATAGGATCCGAGAAAGGTGAAGAAAAGGGA \\
\hline BOP2-4kb-Nco-R 1 & TCACCATGGCTTCAAGATTGCTCATCTT \\
\hline \multicolumn{2}{|l|}{ qPCR } \\
\hline LFY qPCR-F1 & TCTAGACGCCGTCATTTGCTA \\
\hline LFY qPCR-R1 & CCTCAGATAACCCTTCTTGGG \\
\hline AP1 qPCR-LF & TCCACTGATTCTTGTATGGAGAAG \\
\hline AP1 qPCR-RP2 & TCTTCCCCAAGATAATGCCTCTGGT \\
\hline agl24 P15 & GAGGCTTTGGAGACAGAGTCGGTGA \\
\hline agl24 P16 & AGATGGAAGCCCAAGCTTCAGGGAA \\
\hline SOC1 qPCR-F2 & GCTCCAATATGCAAGATACCATAG \\
\hline SOC1 qPCR-R2 & CCCAAGAGTTTACGTTTAGAAGC \\
\hline FUL qPCR-F1 & CTCCAGAAGAAGGATAAAGCCT \\
\hline FUL qPCR-R1 & ACAAAGCCATCTCTGGAGGAG \\
\hline CAL qPCR-F1 & GCTTACCAAACAGATAAAGGAGAG \\
\hline CAL qPCR-R1 & GGTACAAACCACCCATATTTAGG \\
\hline \multicolumn{2}{|l|}{ In situ probes } \\
\hline AP1-LF & TCCACTGATTCTTGTATGGAGAAG \\
\hline AP1-T7-RR & CATAATACGACTCACTATAGGTATGATGATATAAGAACATCGAACATTTG \\
\hline LFY-LF & GATCCTGAAGGTTTCACGAGTGGC \\
\hline LFY-T7-RR & CATAATACGACTCACTATAGGCAGTGGAGAGCGTAACAGTGAACG \\
\hline AGL24 RT-F & ATGGCGAGAGAGAAGATAAGGATAAAG \\
\hline AGL24 T7-RP2 & CATAATACGACTCACTATAGGGACCAATAACACGTACAATATCTGAAAC \\
\hline SOC1-LF & ATGGTGAGGGGCAAAACTCAGAT \\
\hline SOC1-T7-RR & CATAATACGACTCACTATAGGGAGGGAAGAAAGCTAAATTGATACATG \\
\hline FUL-RT-F1 & TCATTTCAGGGTTGTCGTTTCT \\
\hline FUL-T7-R1 & CATAATACGACTCACTATAGGGGACAACGGAGTTCCATCATACAT \\
\hline
\end{tabular}


Table 2.2 Primers for qPCR of ChIP products

\begin{tabular}{ll}
\hline Primer & Sequence 5'-3' \\
\hline AP-1F & CAATATATATGGATATAACGTACAAAC \\
AP-1R & GATGATCAGGACAAAAATCAGTTG \\
AP-2F & TATTTTGGTTGGTTCAGATTTTGTT \\
AP-2R & ACTGGTCCTTCCCCAAGTGT \\
AP-3F & CACGAGACGTCGATAATCAAATTG \\
AP-3R & GGTTTCTTTAGGATTTGCGTGTCG \\
AP-4F & AAAAGGATCAAAAATGGGAAGGGG \\
AP-4R & CCCTTATGGGAGAAGACAACAAGAGC \\
& \\
ACTIN-F & CGTTTCGCTTTCCTTAGTGTTAGCT \\
ACTIN-R & AGCGAACGGATCTAGAGACTCACCTTG \\
\end{tabular}


Table 2.3 Quantitative analysis of floral meristem identity phenotypes in wild-type and mutants

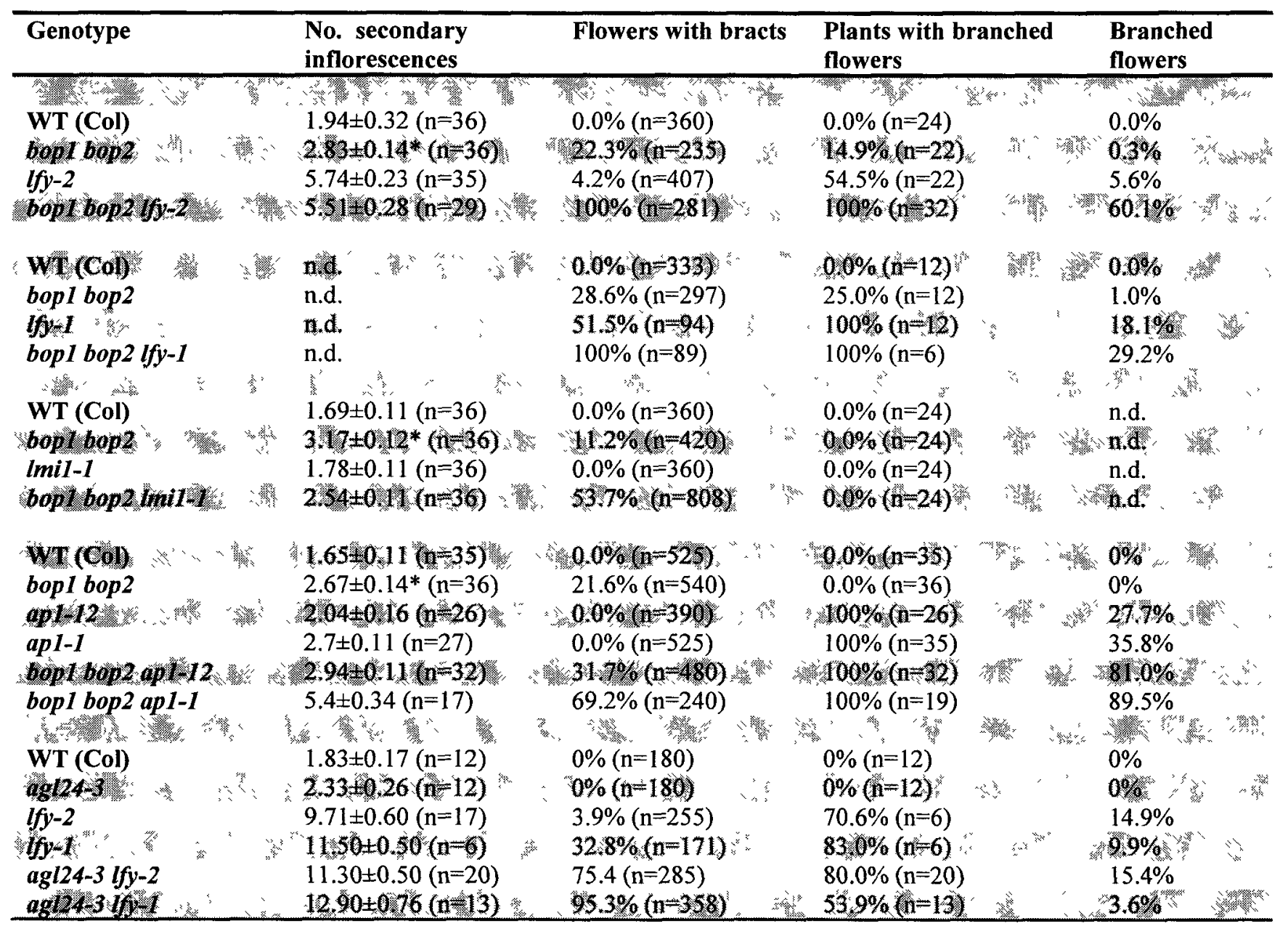

*Significantly different from WT as determined by a Student's t-test, $\mathrm{p}<0.05$. 


\section{Table 2.4 Quantitative analysis of floral-organ identity phenotypes in wild-type and}

\section{mutants}

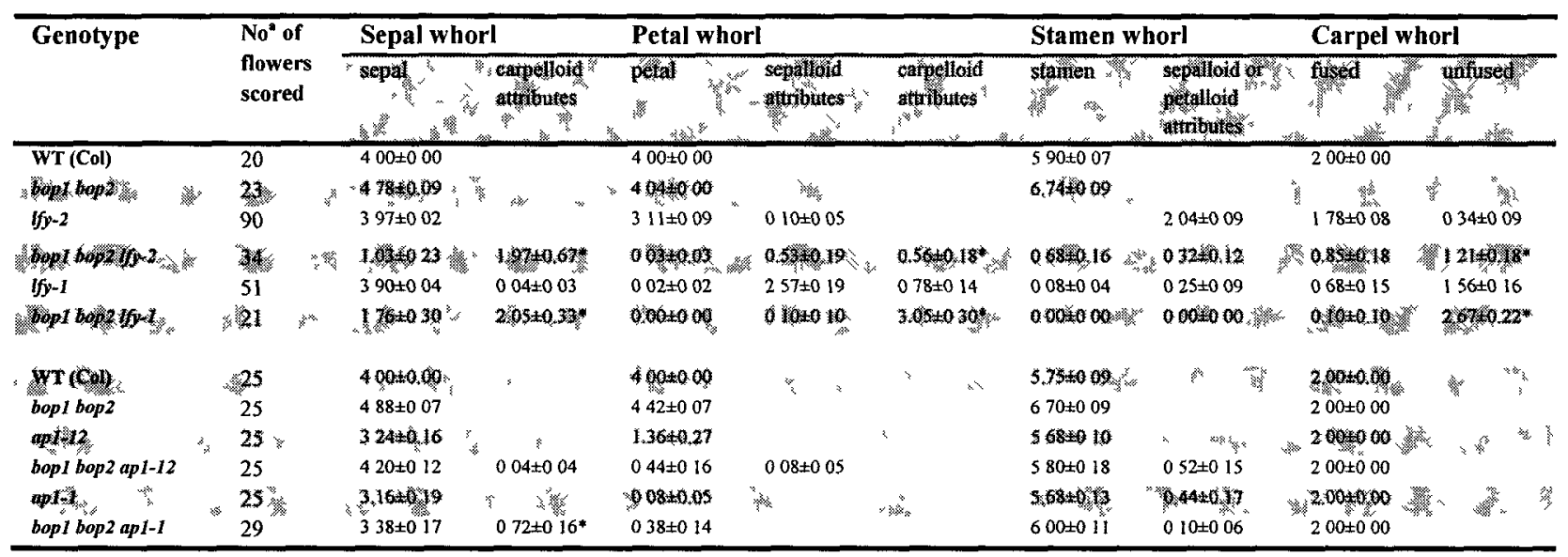

${ }^{a}$ Flowers from nodes 1-15 were scored

* Significantly different from the lfy parental control as determined using a Student's t-test, $\mathrm{p}<0.05$. 
Table 2.5 Quantitative analysis of floral branching and bract suppression by loss-offunction $a g l 24$

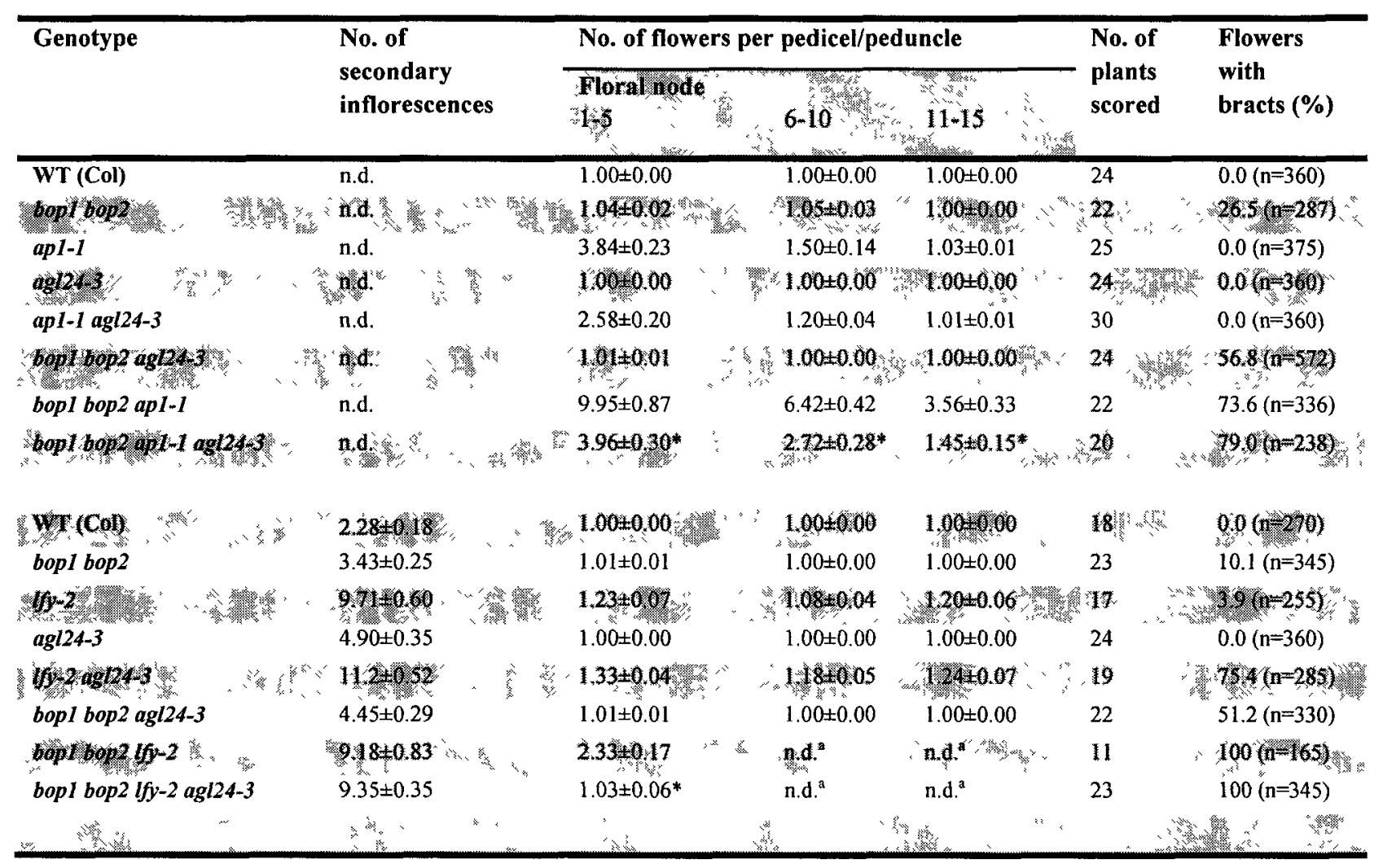

"Significantly different from the triple mutant control as determined using a Student's t-test, $\mathrm{p}<0.05$.

${ }^{\mathrm{a}}$ Floral branching could not be accurately scored due to presence of nodes without a flower (bract only): bop1 bop2 lfy-2 $(21.4 \%, \mathrm{n}=121)$, bopl bop2 lfy-2 agl24-3 $(49.36 \%, \mathrm{n}=235)$ 

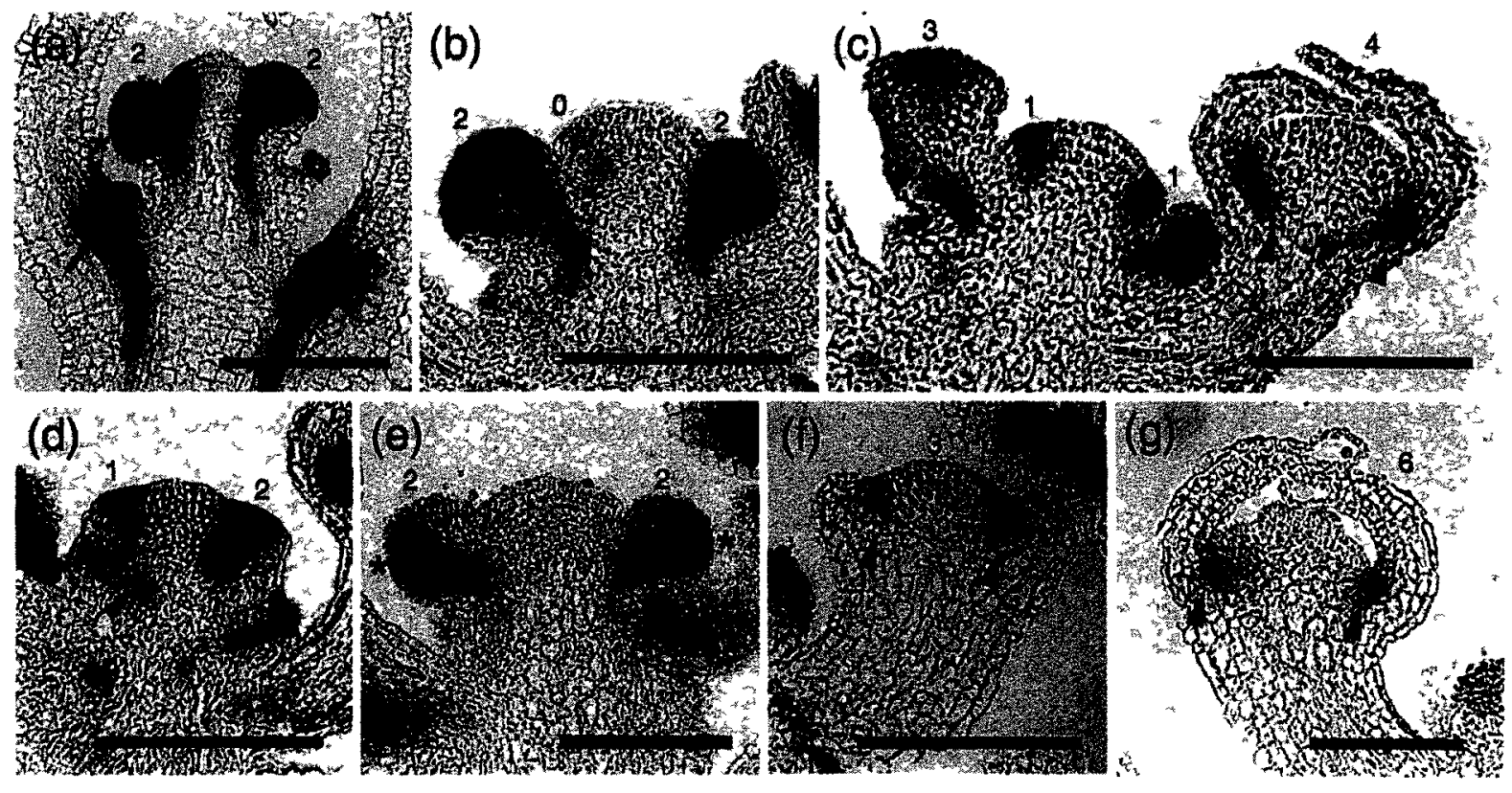

Figure 2.1 BOP2::GUS expression in inflorescence apices.

(a) 14-day-old seedling; expression at the boundary of a lateral shoot meristem (arrow).

(b-e) Expression begins in floral anlagen (0) and associates with the adaxial part of floral meristems until late stage 2 when expression shifts to the floral meristem/bract boundary (asterisks).

$(\mathbf{c}, \mathbf{f}, \mathbf{g})$ Expression is in the dome of early stage 3 flowers but shifts to sepal axils (arrowheads). Numbers in panels indicate floral stage. Scale bars, $100 \mu \mathrm{m}$ 
(a)

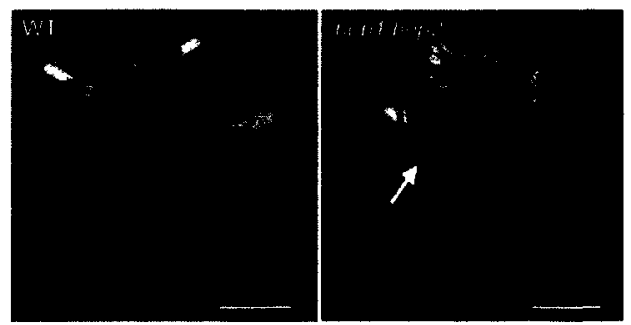

(b)

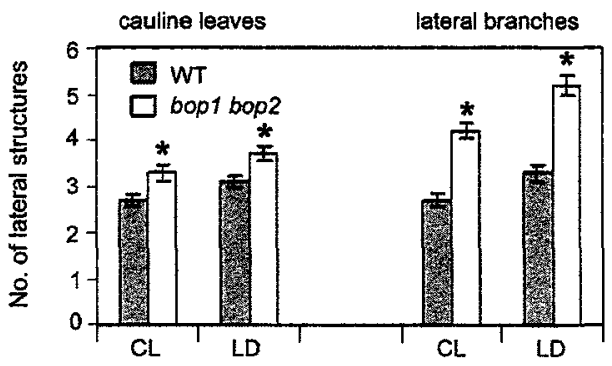

(c)

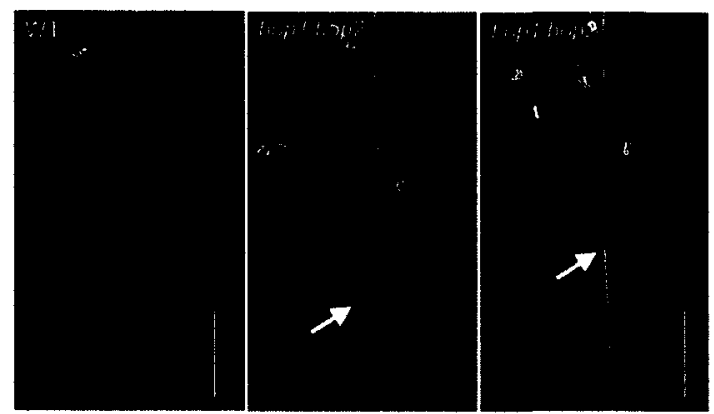

(d)

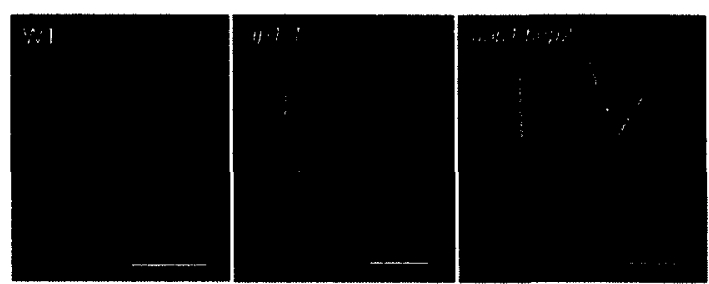

Figure 2.2 Analysis of floral meristem identity defects in bop1 bop2 mutants.

(a) Flowers in bop1 bop2 plants often have a bract (arrow). Scale bars, $2 \mathrm{~cm}$.

(b) Graph showing that lateral branches are more numerous in bop1 bop 2 mutants relative to WT in continuous light (CL) or long-day (LD) photoperiods and that not all branches have a cauline leaf. Asterisks indicate a significant difference from WT (Student's t-test, $\mathrm{p}<0.05$ ). Error bars indicate s.e.m.

(c) Cauline leaves may be absent or displaced at transitional nodes in bopl bop2 plants (arrows). Scale bars, $0.5 \mathrm{~cm}$.

(d) Siliques of WT, ap 1-1, and bop1 bop2 mutants. Scale bars, $1 \mathrm{~cm}$. 
Figure 2.3 Mutation of bop1 bop2 enhances lfy and Imil-1 floral defects.

(a) $l f y-2$.

(b) bop1 bop2 lfy-2; enhanced branching complexity (arrow).

(c) SEM of bop 1 bop 2 lfy-2 flower; carpelloid sepals and internode elongation between floral organs (arrow).

(d) Comparison of floral bracts in bop1 bop2, lfy-2, and bopl bop2 lfy-2 mutants.

(e-f) Comparison of lfy-1 and bop1 bop 2 lfy-1 inflorescences; bracts are larger and more numerous (arrows).

(g) bopl bop 2 lfy-2 bract-only nodes.

(h, j-n) Representative flowers of the indicated genotypes. Ectopic stigmatic papillae and ovules and unfused carpels are more prevalent in bop1 bop 2 lfy-2 and bopl bop 2 lfy-1 flowers compared with $l f y-2$ or $l f y-1$ respectively.

(i) Misexpression of $A G$ in the perianth whorls of bopl bop2 lfy-2 triple mutant flowers.

(o-r) Representative inflorescences of the indicated genotypes; bract formation in bop1 bop2 is enhanced by $l$ mil (arrows). Scale bars: $1 \mathrm{~mm}$ except a-b, d, $5 \mathrm{~mm}$; c, $0.5 \mathrm{~mm}$; i, $100 \mu \mathrm{m}$; o-r, 2 $\mathrm{cm}$. 


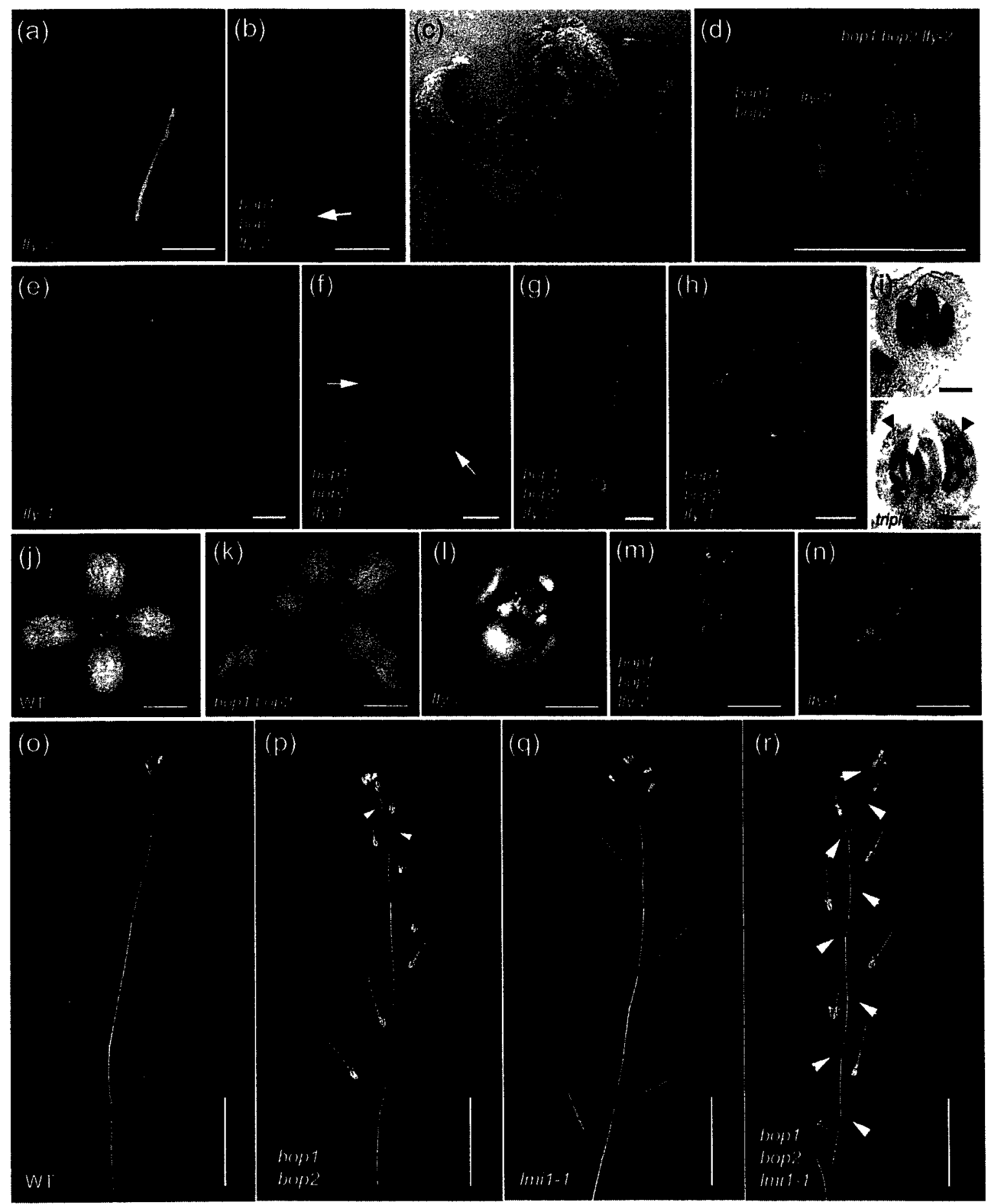


Figure 2.4 Mutation of bop1 bop2 enhances ap1 floral defects.

(a-d) Representative inflorescences for the genotypes indicated. Arrows denote node of first flower. Floral nodes in bop1 bop 2 apl-I are highly branched and often indeterminate.

(e) Curd-like bop1 bop2 ap1-1 inflorescence apex.

Comparison of floral architectures:

(f) bop1 bop2; most flowers are unbranched, abaxial sepals are petalloid.

(g) ap 1-1; secondary flowers in the axils of first-whorl floral organs.

(h) bop1 bop 2 ap 1-1 floral shoot with determinate architecture. Large leafy sepal/bracts subtend flowers (arrows).

(i) Late stage bopl bop2 ap1-1 floral node showing complex branching architecture.

(j) Early stage bop1 bop2 ap 1-1 floral node; internode elongation between first-whorl organs and floral bracts (arrow).

(k) bop1 bop 2 ap1-1 flower; arrows indicate ovules on the margin of carpelloid sepals.

(l) ap 1-12 flower; weak allele.

(m) bop1 bop2 ap1-12 flower; similar to ap1-1. Scale bars: a-d, $2.5 \mathrm{~cm}$; e-g, $2 \mathrm{~mm}$; h, $0.5 \mathrm{~cm}$; i$\mathrm{m}, 1 \mathrm{~mm}$. 


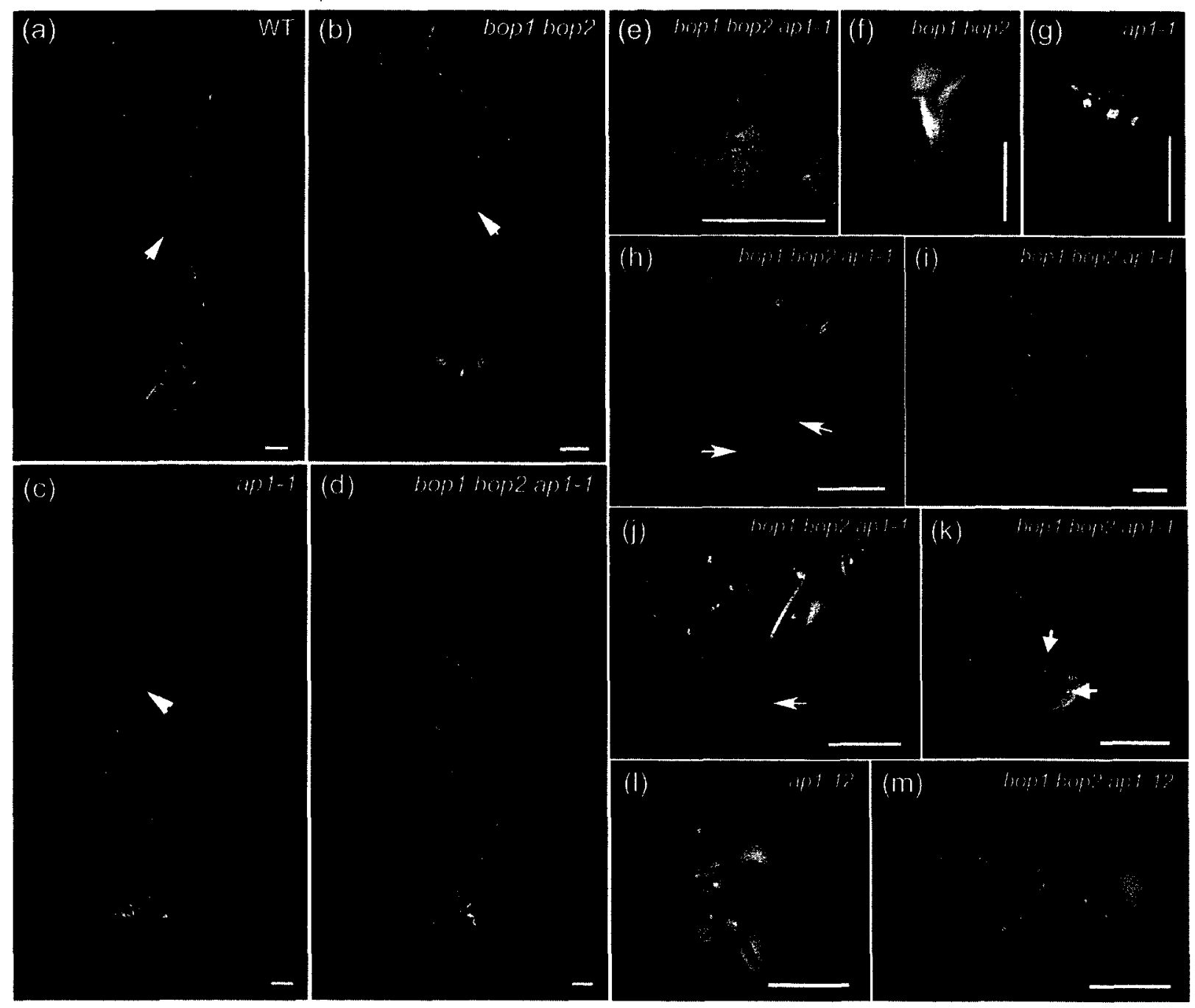



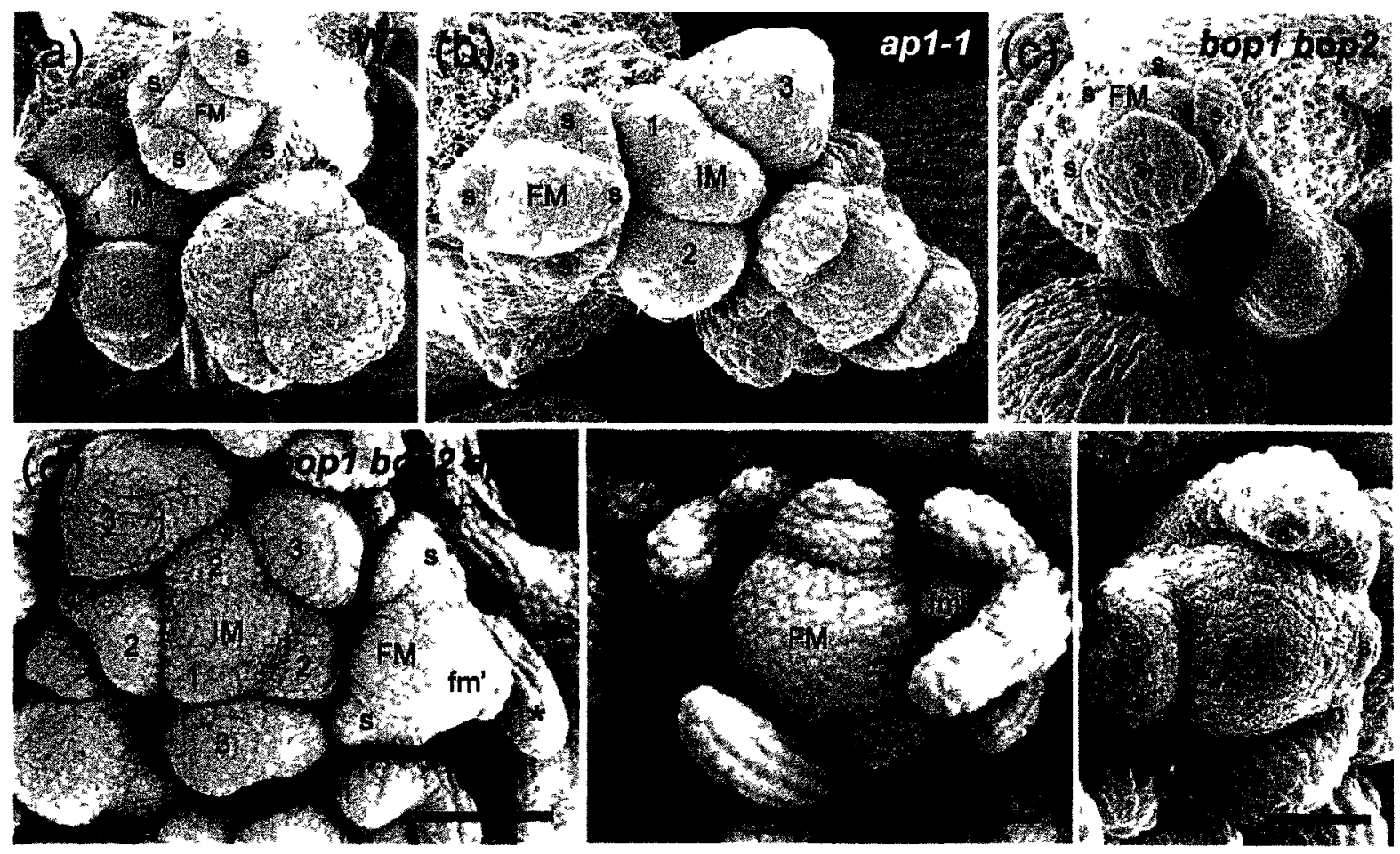

Figure 2.5 Scanning electron micrographs of WT and mutant inflorescence apices.

(a) WT control.

(b) ap 1-1.

(c) bop1 bop2; asterisk denotes floral bract.

(d) bop1 bop2 ap 1-1; asterisks denote floral bracts. Note the aberrant morphology of stage 3 primordia lacking clearly defined sepals. Ectopic axillary flowers (fm') are first evident in late stage 3 structures.

(e) bop1 bop 2 ap 1-1; late stage 3 flower showing large leafy bracts and a secondary floral meristem (fm') developing in the axil of a first whorl bract/sepal.

(f) bop1 bop 2 ap1-1; stage 3 structure showing spiral initiation of first whorl organs. Numbers in panels indicate floral stages. Scale bars, $100 \mu \mathrm{m}$ except $50 \mu \mathrm{m}$ in (f). IM, inflorescence meristem. FM, floral meristem. s, sepal. 


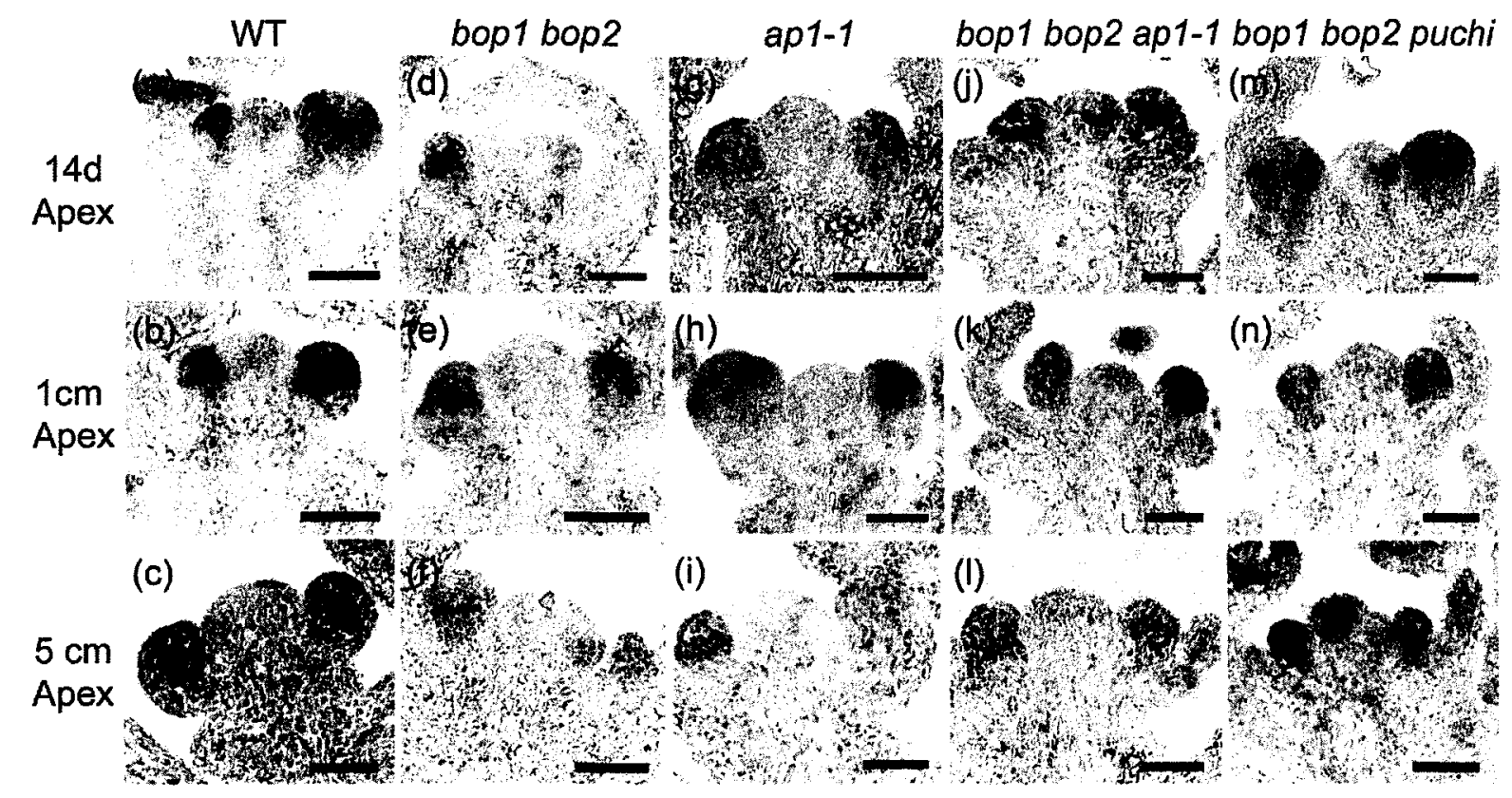

Figure 2.6 Expression of $L F Y$ in WT and mutant inflorescence apices.

Inflorescence apices sectioned from 14-day old seedlings or plants with 1-cm and 5-cm bolts were hybridized with a $L F Y$ anti-sense probe.

(a-c) WT control.

(d-f) bop1 bop2 apices.

(g-i) ap 1-1.

(j-1) bop1 bop2 ap1-1.

(m-o) bopI bop 2 puchi control. LFY expression in all genotypes is similar to that in ap1-1 mutants. Scale bars, $100 \mu \mathrm{m}$. 
Figure 2.7 In situ analysis of $A P 1$ expression in WT and mutant apices.

Inflorescence apices from $1-\mathrm{cm}$ or $5-\mathrm{cm}$ bolts were examined.

(a-c) WT.

(d-f) bop1 bop2.

(g-i) $l f y-2$.

(j-l) bopl bop2 lfy-2; API transcript reduced relative to lfy-2.

(m-o) lfy-1. Arrows indicate expression in stage 2-3 flowers.

(p-r) bop1 bop2 lfy-1; no detectable AP1 transcript.

(s-u) bopl bop 2 puchi control. Scale bars, $50 \mu \mathrm{m}$. 
$1 \mathrm{~cm}$ bolt

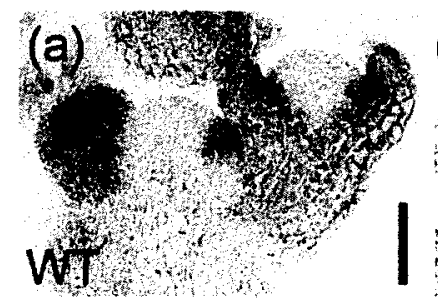

(⿻)
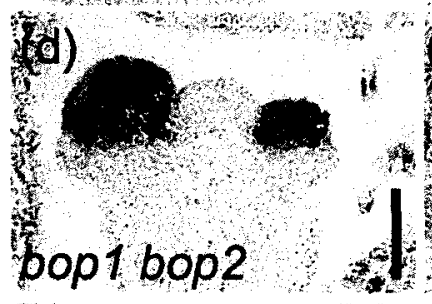

(b)

$5 \mathrm{~cm}$ bolt

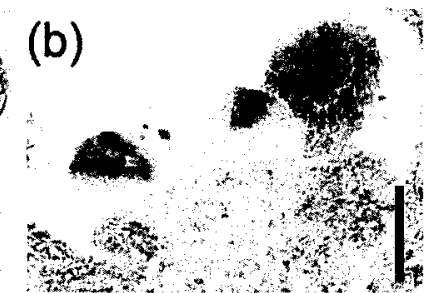

(f)
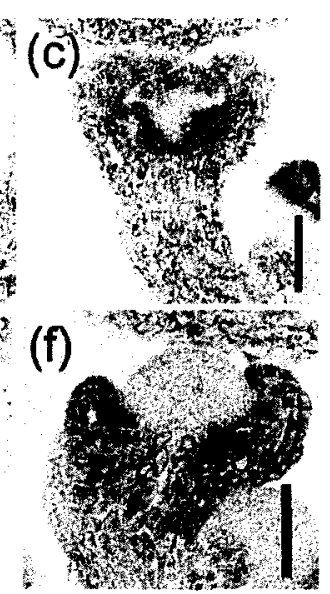

(g)

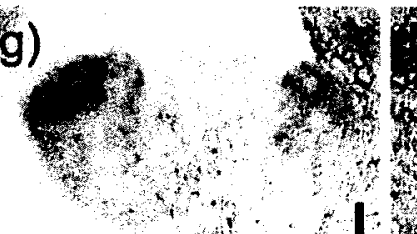

Ify-2, 9

(j)

?

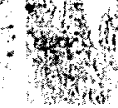

(k)

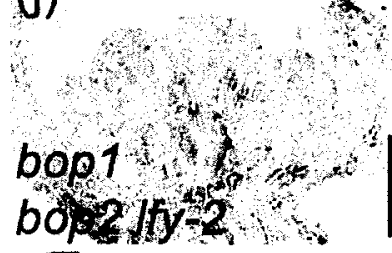

(m)

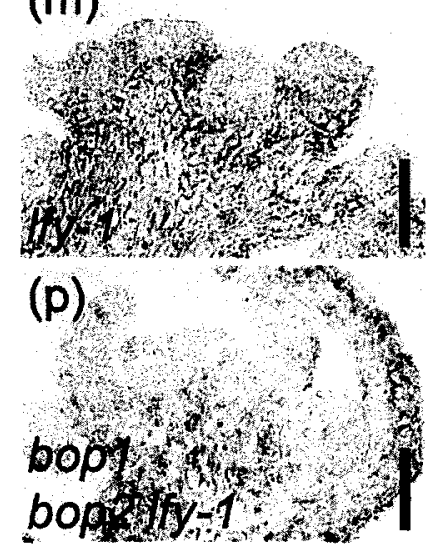

(n)

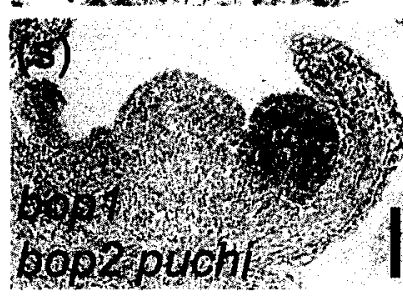

(9)

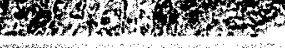

,
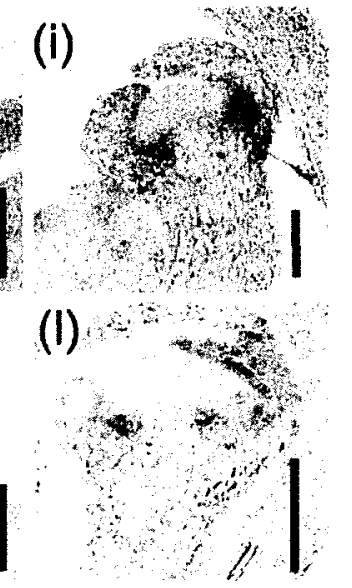

(o)

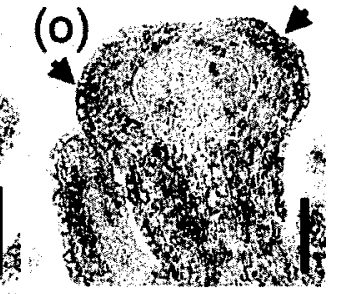

(r)

(r), 3

$\begin{array}{llll} & \\ 3\end{array}$ W

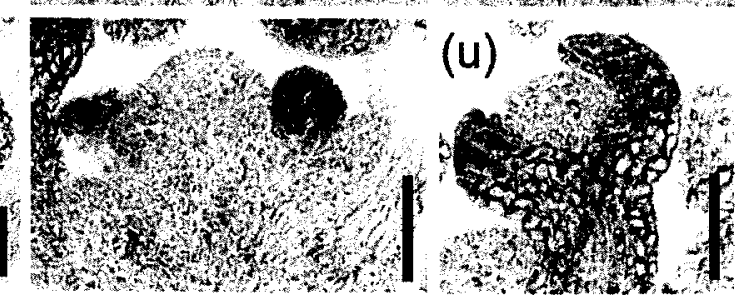


(a)

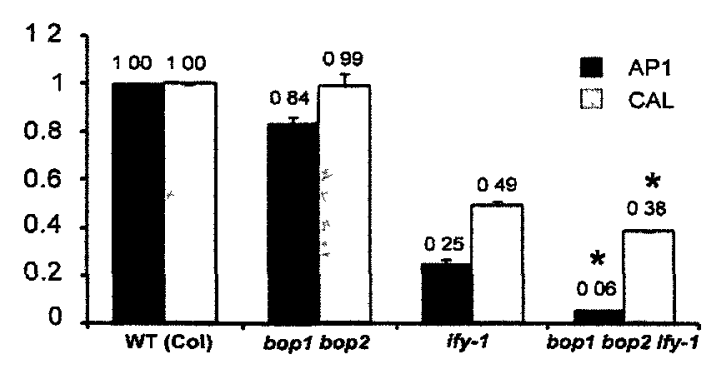

(b)

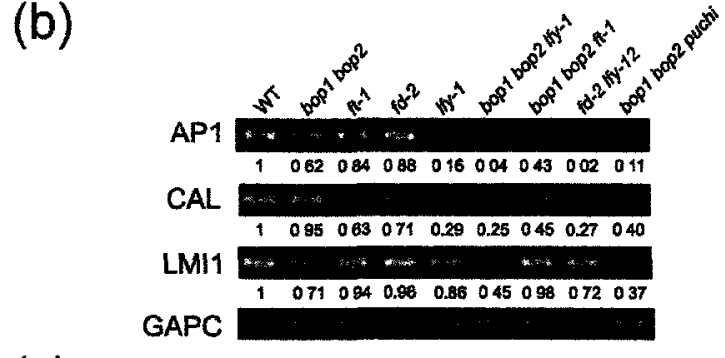

(c)

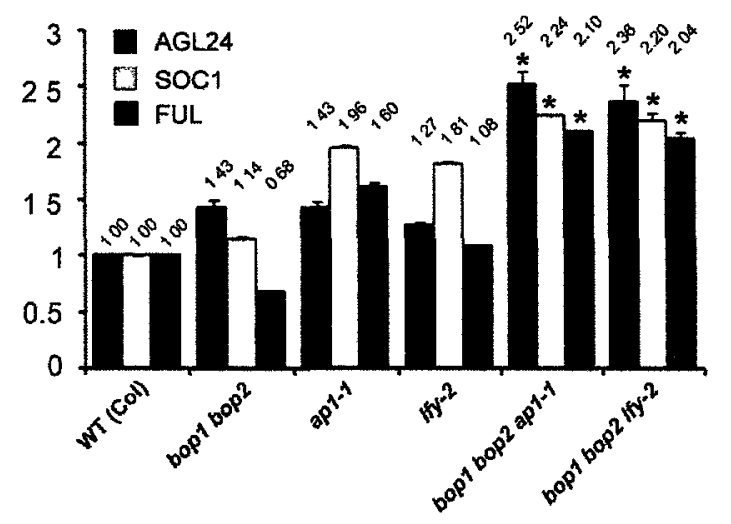

Figure 2.8 Quantitative analysis of $A P 1, C A L$, and flowering-time gene expression in WT and mutant apices.

(a) Relative $A P 1$ and $C A L$ transcript levels in apices of 5 -cm bolts. $A P I$ transcript is significantly lower in plants lacking both BOP and LFY activities.

(b) AP1 transcript levels in the apices of 1-cm bolts for genotypes as indicated. Loss-of-function bop1 bop 2 lowers AP1 transcript accumulation in both lfy and $f t$ mutants.

(c) Relative AGL24, SOC1, and FUL transcript levels in apices of 5-cm bolts for the indicated genotypes. Transcript levels are highest in triple mutant apices. Asterisks in (a) and (c) indicate that values are significantly different from parental controls (Student's t-test, $\mathrm{p}<0.05$ ). Values represent mean. Error bars indicate s.e.m. 
Figure 2.9 In situ analysis of $\boldsymbol{A G L 2 4}$ expression in WT and mutant inflorescence apices. (a-c) WT control; transcript localizes to the cryptic bract (arrows) at late stage 2.

(d-e) bop1 bop2; enlarged bracts (arrows) and misexpression in the dome of stage 3 flowers (asterisk).

(f-g) ap1-1; ectopic expression in the dome of stage 2 flowers and in stage 3 sepals (arrows).

(h-i) lfy-2; ectopic expression in the dome of stage 3 flowers.

(j-k) bop1 bop2 ap1-1; enhanced misexpression.

(1-m) bop1 bop2 lfy-2; enhanced misexpression. Numbers in panels indicate floral stage. Scale bars, $50 \mu \mathrm{m}$. 

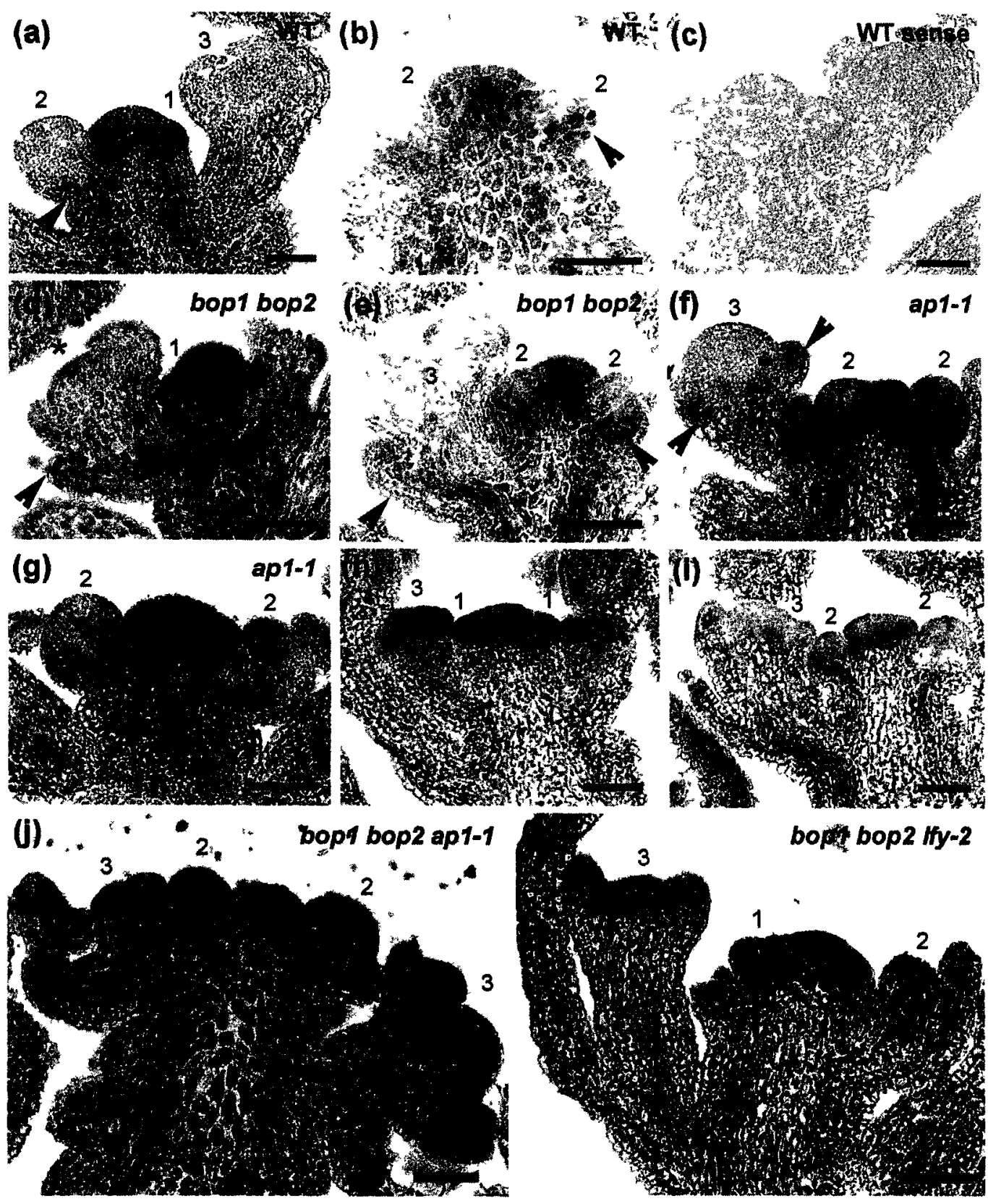


\section{Figure 2.10 Expression of $S O C 1$ in WT and mutant inflorescence apices.}

Inflorescence apices sectioned from plants with $5-\mathrm{cm}$ bolts were hybridized with a $S O C 1$ antisense probe.

(a-b) WT control apices; $\mathrm{SOCl}$ is expressed throughout the inflorescence meristem and stage 1 flowers but clears from the dome of the primordia in late stage 2 and is restricted to the cryptic bract. Expression resumes in early stage 3 in the center of the floral meristem and expands in the interior of the flower but is excluded from the developing sepals.

(c-d) bop1 bop2 apices; resembles wild-type.

(e-f) ap1-1 apices; SOC1 expression clears from the dome of the primordia in late stage 2 but is ectopically expressed in the sepal whorl of stage 3 flowers (arrow).

(g-h) lfy-2 apices; resembles wild-type.

(i-j) bopl bop 2 lfy-2 apices; expression of SOCl fails to clear from the dome of stage 2 primordia and is upregulated throughout stage 3 flowers.

(k-l) bop1 bop2 ap1-1apices; expression of SOC1 fails to clear from the dome of stage 2 primordia and is upregulated throughout stage 3 flowers. Numbers in panels indicate floral stage. Scale bars, $100 \mu \mathrm{m}$. 

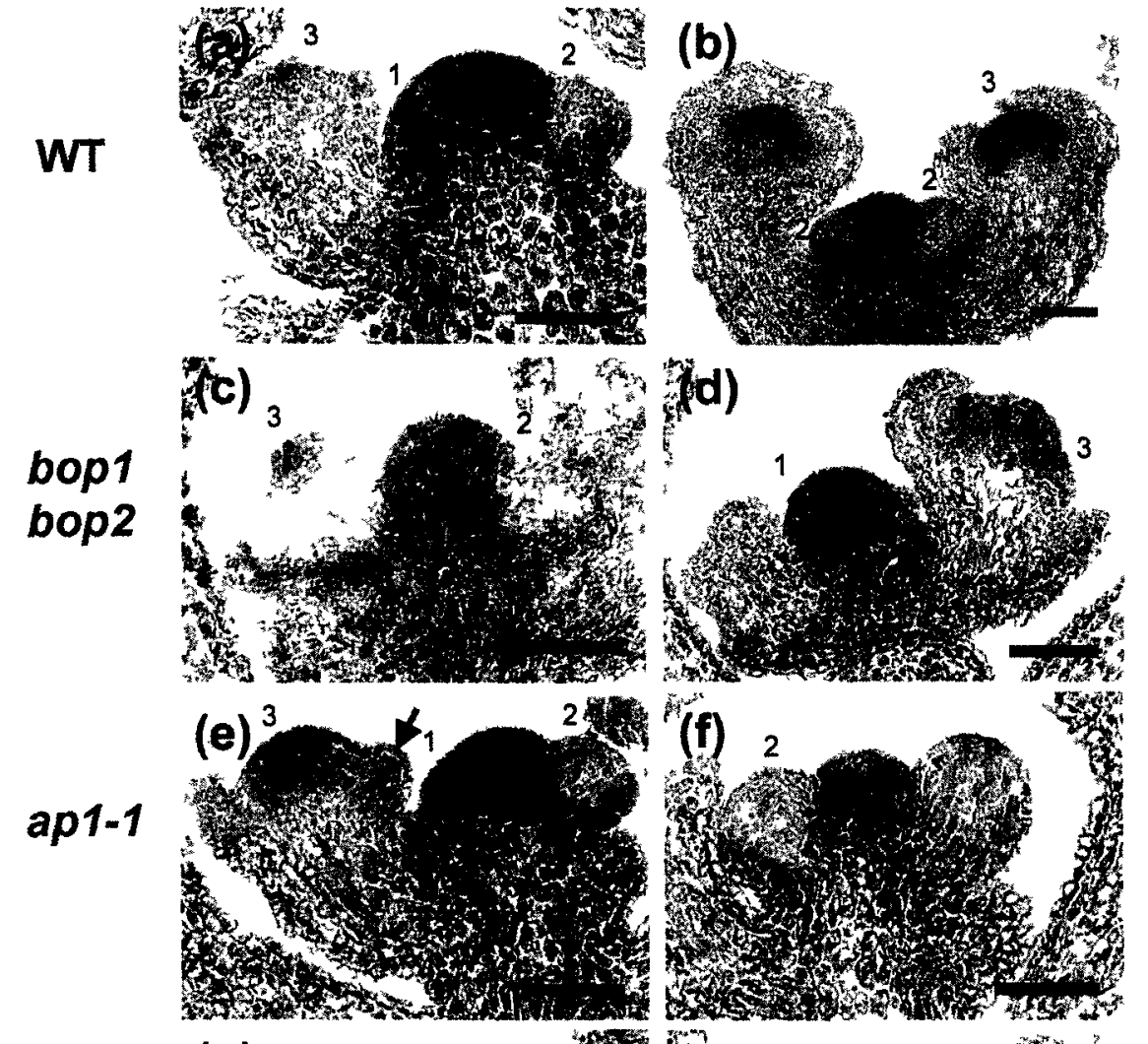

Ify-2

(g)
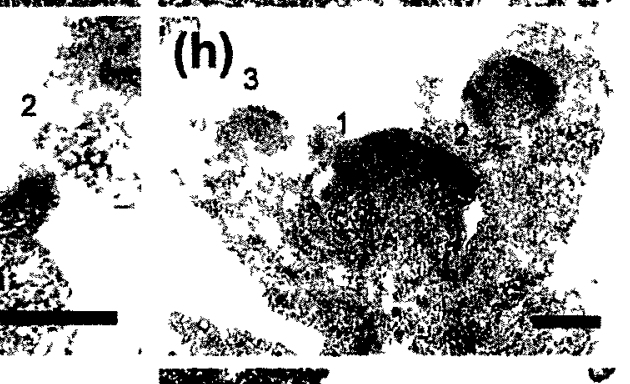

(i)

bop1 bop2 Ify-2
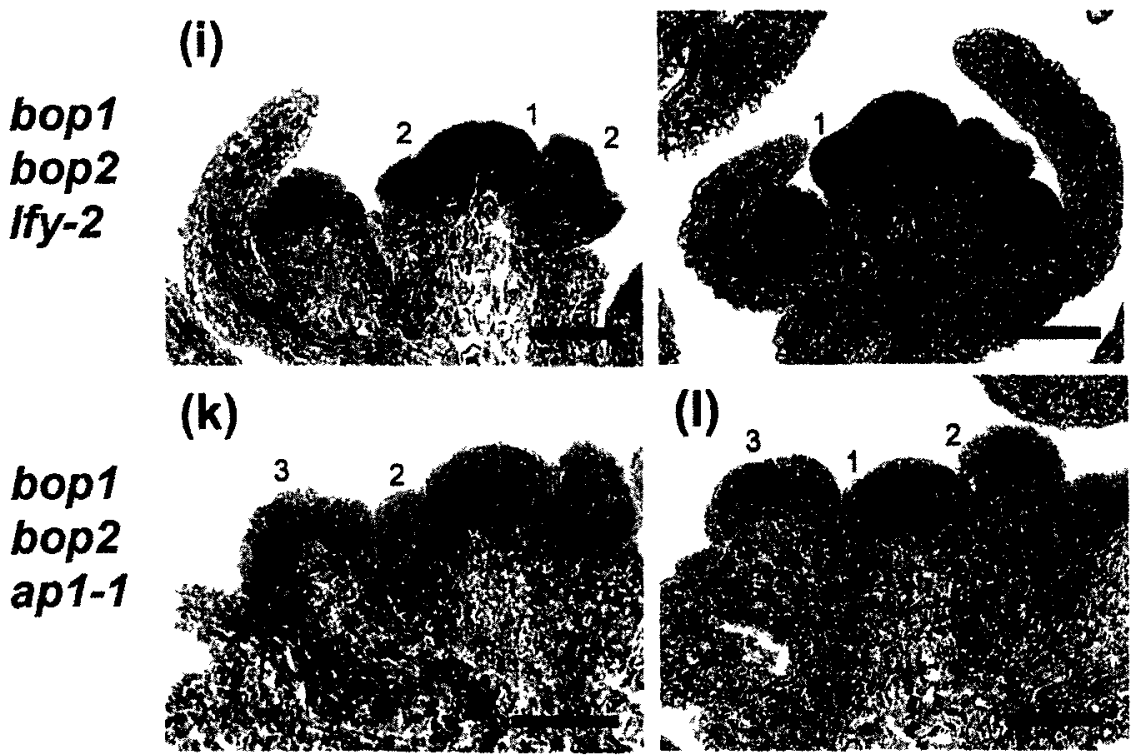

(I)

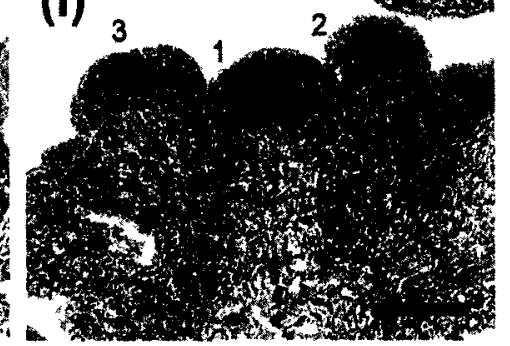




\section{Figure 2.11 Expression of $F U L$ in $W T$ and mutant inflorescence apices.}

(a-c) WT control apices; FUL is expressed throughout the inflorescence meristem and stage 1 flowers but clears from the dome of the primordia in late stage 2 and is restricted to the cryptic bract (arrow). Expression resumes in the center of the floral meristem in stage 3.

(d-f) bop1 bop 2 apices; FUL expression persists in the dome of primordia at late stage 2 (arrows) but expression at stage 3 appears normal.

(g-i) apl-1 apices; FUL expression fails to clear from the dome of stage 2 primordia and is expanded in the central part of stage 3 flowers (arrows).

(j-l) lfy-2 apices; $F U L$ expression persists in the dome of stage 2 flowers.

(m-o) bopl bop 2 ap 1-1 apices. FUL is expressed throughout stage 2 and early stage 3 structures and its domain of expression is expanded in stage 3 flowers.

(p-r) bopl bop 2 lfy-2 apices; FUL is expressed throughout stage 2 and early stage 3 structures and its domain of expression is expanded in stage 3 flowers. Numbers in panels indicate floral stage. Scale bars, $100 \mu \mathrm{m}$ 


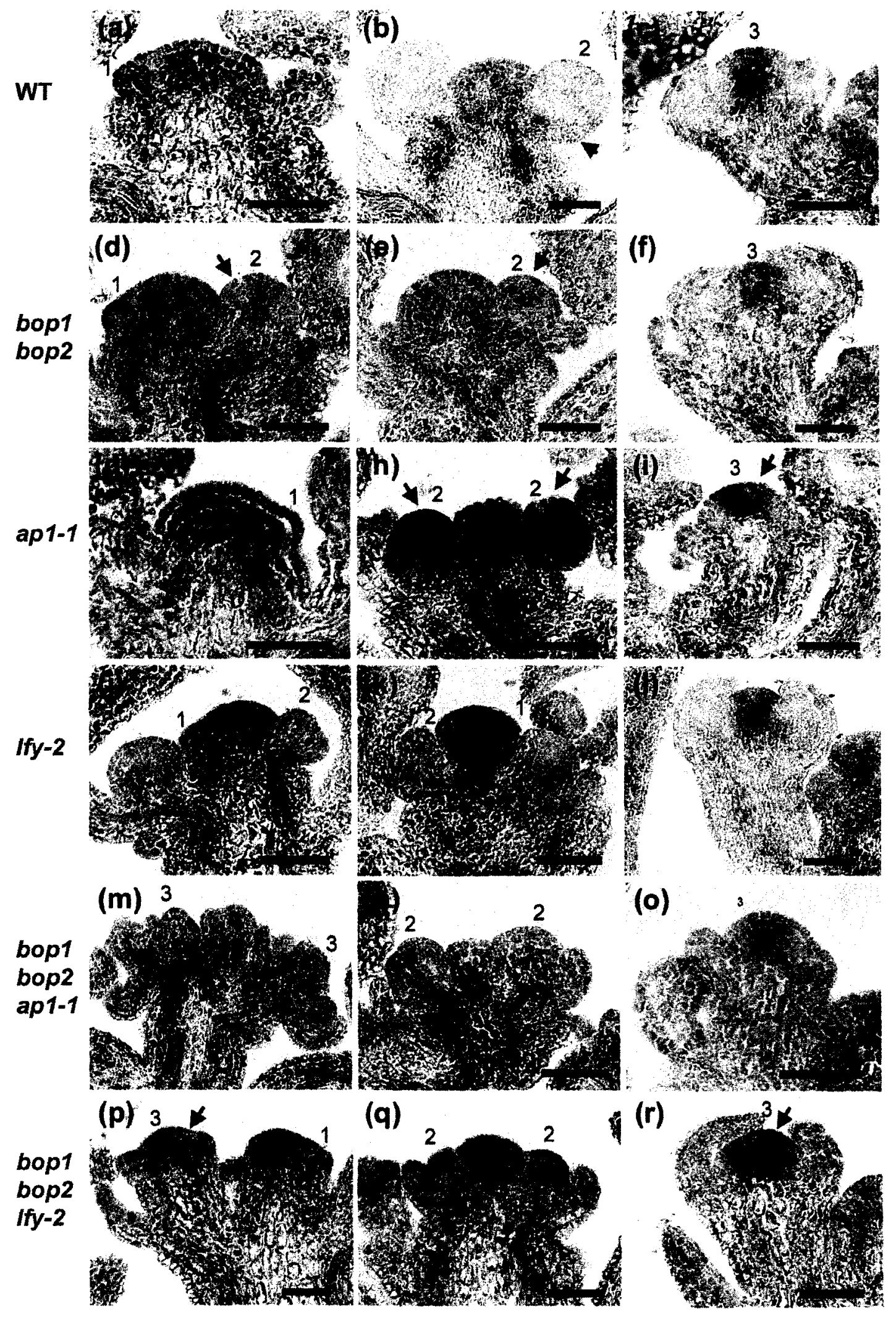




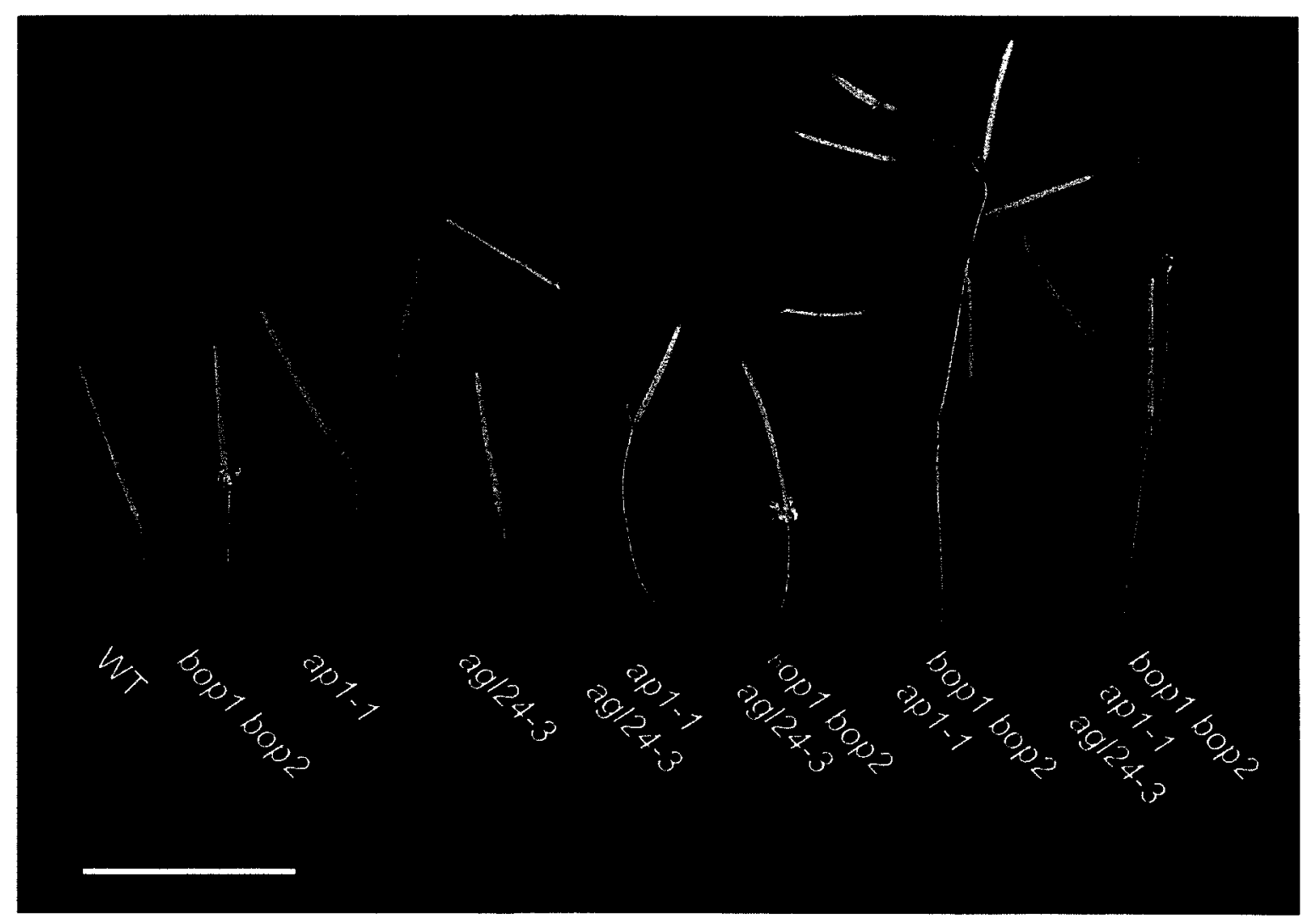

Figure 2.12 Floral branching in bop1 bop2 ap 1-1 triple mutants is suppressed by agl24. Representative shoots (nodes 6 to 10) are shown for the indicated genotypes. Scale bar, $2 \mathrm{~cm}$. 


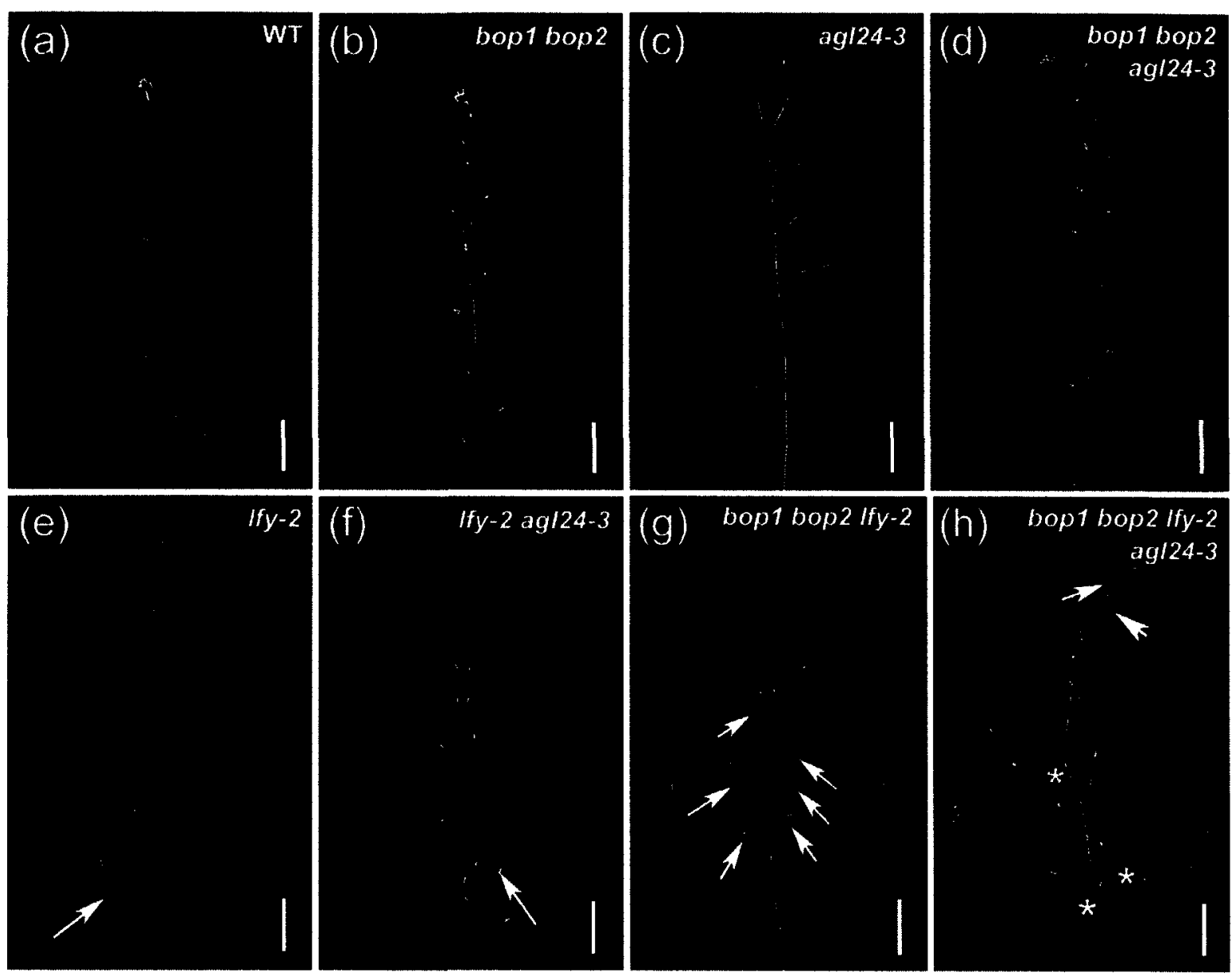

Figure 2.13 Floral branching in bop1 bop 2 lfy-2 triple mutants is suppressed by loss-offunction agl24.

Inflorescence apices from:

(a) WT control plant.

(b) bop1 bop 2 mutant.

(c) agl24-3 mutant.

(d) bop1 bop2 agl24-3 mutant.

(e) lfy-2 mutant, arrow indicates a branched flower.

(f) lfy-2 agl24-3 mutant, arrow indicates a branched flower.

(g) bop1 bop 2 lfy-2 mutant, arrows indicate branched flowers.

(h) bopl bop2 lfy-2 agl24-3 mutant, asterisks indicate bracts and arrows indicate nodes lacking a flower (bract only). Scale bars, $2 \mathrm{~cm}$. 

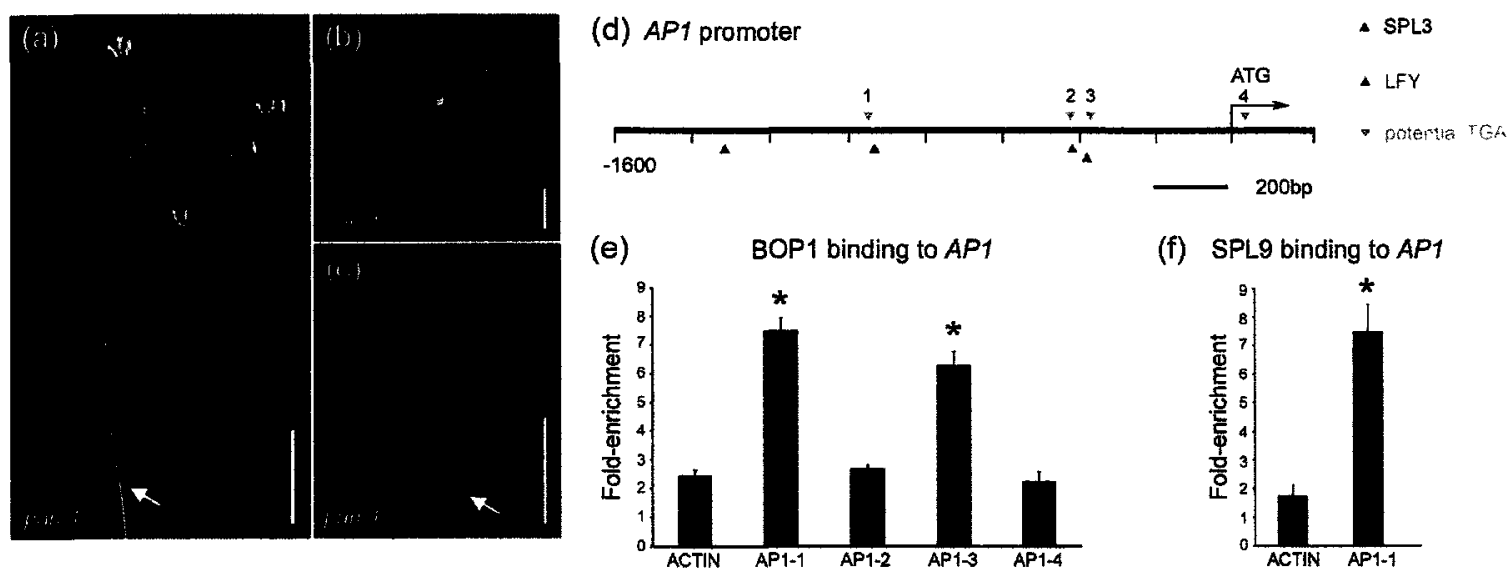

(f) SPL9 binding to $A P 1$

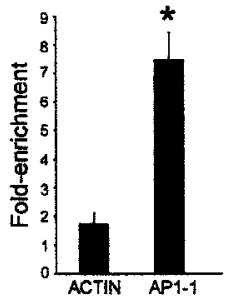

Figure 2.14 BOP1 binds in vivo to sites containing TGA binding motifs in the AP1 promoter.

Floral meristem identity defects in pan-1 mutants:

(a) Cauline leaf absent at transitional node (arrow). Scale bar, $2 \mathrm{~cm}$.

(b) Branched flowers.

(c) Flower with bract. Scale bars, $5 \mathrm{~mm}$.

(d) Scale diagram of the $A P 1$ promoter indicating the binding sites of direct regulators and potential TGA binding motifs. Numbers indicate regions tested for BOP1-GFP occupancy in ChIP assays (e-f). Binding site locations derived from: Wang et al., 2009; Wigge et al., 2005; Yamaguchi et al., 2009; Wang et al., 2009. See text for identification of potential TGA binding sites.

(e) BOP1-GFP occupancy at AP1 promoter sites.

(f) GFP-SPL9 occupancy at $A P 1$ promoter site 1 . Asterisks in e and f indicate significantly different from ACTIN control (Student's t-test, p<0.05). Error bars indicate s.e.m. 


\section{Figure 2.15 Yeast 2-hybrid and BiFC analysis of BOP protein-protein interactions.}

(a) Pair-wise interactions of BOP2 with transcription factors directly regulating $A P 1$ and/or AGL24. Only PAN interacts with BOP2.

(b-j) BiFC analysis of interactions and localization of BOP2 and PAN YFP-tagged fusion proteins. Mesophyll protoplasts were monitored for YFP fluorescence 12-20 h after transfection. Inset panels show the corresponding DIC image under visible light.

(b) No vector background fluorescence control.

(c) Free EYFP localizes to the nucleus and the cytoplasm.

(d) PAN-EYFP fusion protein localizes to the nucleus.

(e-f) BOP2-EYFP fusion protein localizes to the cytoplasm and the nucleus.

(g) Infrequently, the fluorescent signal occurs in punctuate spots throughout the cytoplasm.

(h) BOP2-nEYFP fusion protein shows no signal.

(i) PAN-cEYFP fusion protein shows no signal.

(j) Protoplasts expressing both BOP2-nEYFP and PAN-cEYFP show nuclear localized fluorescence. 

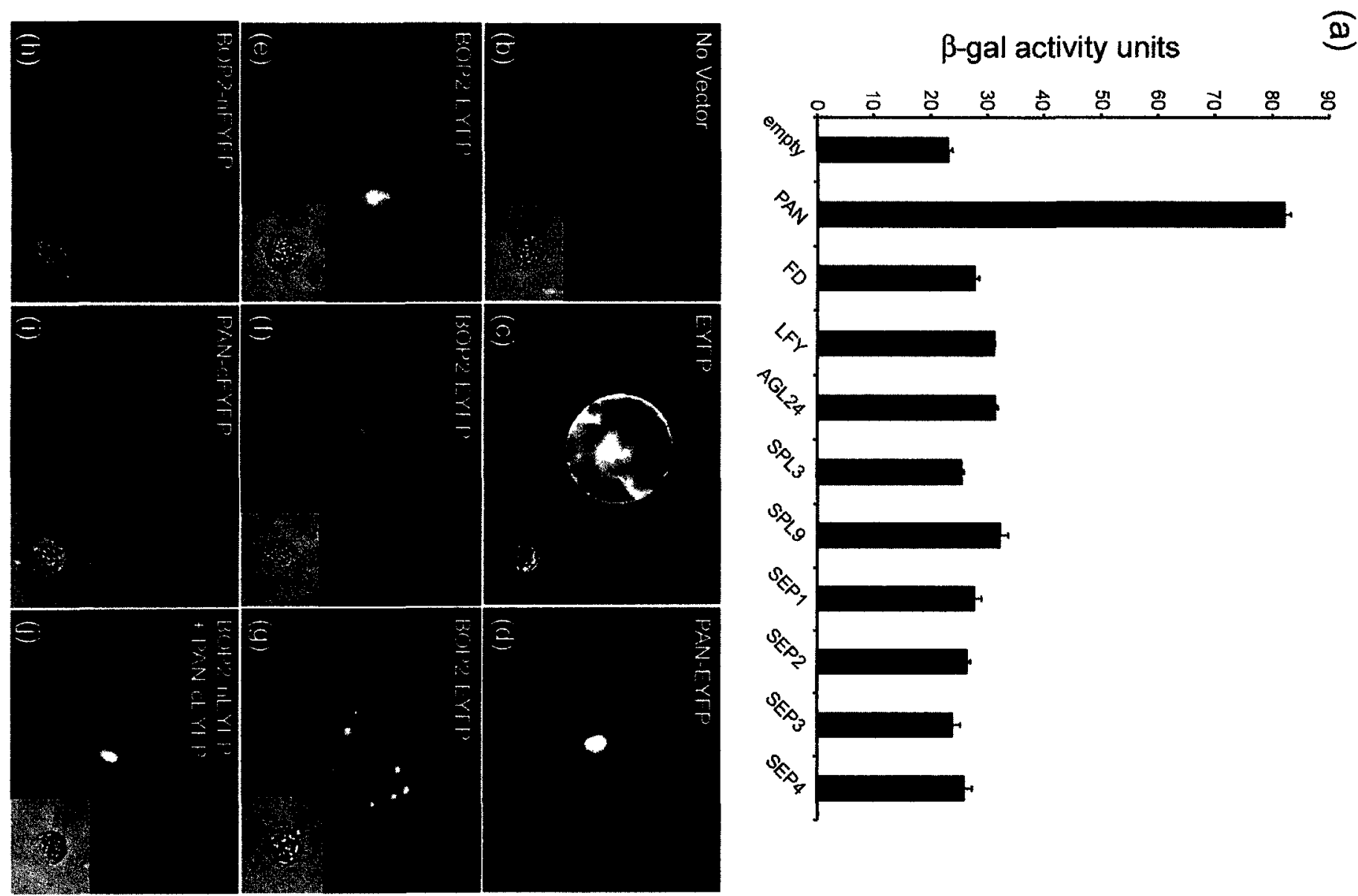


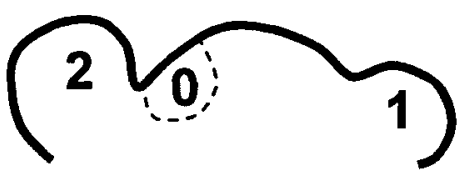

Stage 0-2

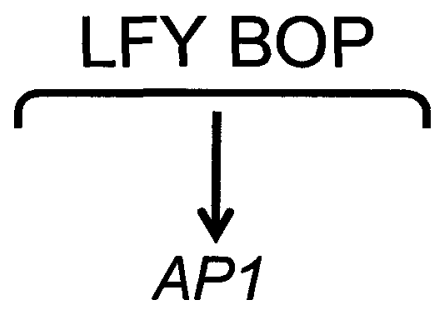

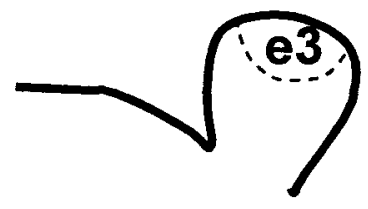

Late 2-Early 3

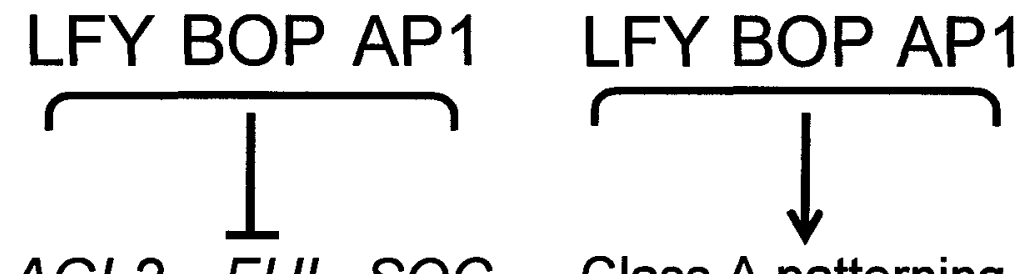

AGL2 FUL SOC Class A patterning

Figure 2.16 Model for BOP promotion of floral meristem identity.

The $B O P$ genes are co-expressed with $L F Y$ in floral anlagen and function distinctly from LFY to upregulate $A P I$ in stage 1 flowers. During stage 2, the overlapping activities of BOP1/2, LFY, and AP1 down-regulate $A G L 24, S O C 1$, and $F U L$ in floral meristems to maintain floral fate. After stage 3, the continued activities of BOP1/2, LFY, and AP1 promote A-class floral patterning. 


\title{
Chapter 3
}

BLADE-ON-PETIOLE1 and 2 function redundantly with

\begin{abstract}
ASYMMETRIC LEAVES1 and 2 to promote simple leaf shape by preventing reactivation of $K N O T T E D I-L I K E H O M E O B O X$ meristem genes in the petiole
\end{abstract}

I provided all of the data in this Chapter with the following exception: Tieqiang $\mathrm{Hu}$ performed the qPCR experiment shown in Figure 3.3. 


\begin{abstract}
Leaves are flat structures specialized for photosynthesis. Leaf shapes are classified into simple and compound forms according to their degree of complexity on the petiole. Three main factors have been shown to promote the transformation of simple leaves to compound leaves: 1) the expression of Class I KNOTTED1-LIKE HOMEOBOX (KNOXI) meristem genes, 2) the expression of CUP-SHAPED COTYLEDON (CUC) boundary genes; and 3) the formation of auxin maxima. A number of factors expressed in leaves including ASYMMETRIC LEAVES1 (AS1), AS2, BLADE-ON-PETIOLE1 (BOP1) and BOP2 have been identified as negative regulators of $K N O X l$ gene expression, but how these genes interact to control the level and spatial pattern of $K N O X 1$ misexpression in leaves has not been investigated. Here, I use a combination of genetic and expression studies to show that BOP $1 / 2$ differentially repress $K N A T 2$ and KNAT6 expression in vegetative apices and that BOP1/2 and AS1-AS2 function synergistically to maintain $K N O X 1$ gene repression in leaf petioles though AS2-dependent and AS2-independent pathways. My data show that the major target of repression in leaves is BREVIPEDICELLUS $(B P)$. Consistent with this, I show that $b p$ mutations partially rescue petiole defects in bopl bop2 as 1 and bopl bop 2 as 2 triple mutants. Collectively, my data support the model that BOP1/2 function redundantly with AS1-AS2 to promote simple leaf shape by maintaining repression of $K N O X I$ meristematic genes in the petiole.
\end{abstract}




\subsection{Introduction}

Land plants display a variety of leaf architectures designed to optimize the capture of light and defend against biotic and abiotic stresses. Although leaves vary dramatically in size and shape, they are classified into two major groups according to their degree of complexity: simple and compound. The major difference between simple and compound leaves is that simple leaves have one undivided blade on the main axis (petiole) whereas compound leaves have several leaves arranged bisymmetrically on the petiole. Though very different in shape, simple and compound leaves can be found in related species of the same genus (reviewed in Hasson et al., 2010; Uchida et al., 2010).

Both simple and compound leaves are initiated from stem cell located in the SAM. SHOOT MERISTEMLESS (STM) from KNOXI family is expressed in the SAM except at the incipient leaf primordia and is one of the master regulators for the establishment and maintenance of SAM (Long et al., 1996). Other KNOX1 members, such as BREVIPEDICELLUS $(B P)$ (also known as KNOTTED-LIKE from ARABIDOPSIS THALIANA or KNAT1) and KNAT6, which are expressed in the PZ of SAM or the primordia-SAM boundary, respectively, also contribute to the maintenance of SAM since loss-of-function mutants $b p$ and knat6 enhance to strong the phenotype of weak stm-2 alleles (Byrne et al., 2002; 2003; BellesBoix et al., 2006). Strikingly, KNAT2 is also expressed in the SAM but its inactivation does not enhance stm-2, knat6 stm-2, or bp knat6 stm-2 phenotypes, making the role of KNAT2 in the SAM unclear (Belles-Boix et al., 2006). KNOX1 homeodomain proteins form hereodimers with BELL homeodomain proteins in vivo (Kanrar et al., 2006) and the BELL protein PENNYWISE (PNY), POUNDFULLISH (PNF), and ARABIDOPSIS THALIANA HOMEOBOX1 (ATH1) also contribute to SAM establishment and maintenance since the pny pnf athl triple mutant also 
lacks of SAM (Rutjens et al., 2009). KNOXI homeodomain proteins function in part via balancing cytokinin and gibberellin levels in plants to establish and maintain the SAM (Rupp et al., 1999; Jasinski et al. 2005; Yanai et al., 2005; reviewed in Barton, 2010; Hay and Tsiantis, 2010). Though each of the $K N O X 1$ genes has a unique expression profile in the SAM, they share a common repression zone--the incipient leaf primordia (Lincoln et al., 1994; Long et al., 1996). This repression zone represents a transition of cell fate (from indeterminate to determinate) and is maintained differently in simple and compound leaf primordia.

In simple leaf species such as Arabidopsis, $K N O X 1$ genes are repressed throughout leaf development. If they are overexpressed, lobed leaves and ectopic meristems in the sinus of these lobes resembling compound leaves are developed (Lincoln et al., 1994; Chuck et al., 1996; Gallois et al., 2002), In the compound leaves of plants such as tomato and Cardamine, KNOXI genes are transiently repressed in the early stages of leaf development but reactivated in later stages of leaf primordia development (Hareven et al., 1996; Hay and Tsiantis, 2006b). Genetic studies show that higher and prolonged expression of KNOXl genes in tomato and Cardamine hirsuta leaves results in a higher order of leaflets (Hareven et al., 1996; Hay and Tsiantis, 2006b) indicating that the temporal and spatial regulation of $K N O X 1$ genes is important for the simplecompound leaf deviation.

A number of genes in Arabidopsis contribute to maintenance of KNOXI repression during leaf development. These include $A S I$ that encodes a MYB transcription factor, $A S 2$ that belongs to the LBD family (Byrne et al., 2000; Ori et al., 2000; Semiarti et al., 2001; Iwakawa et al., 2002; Lin et al., 2003), and BOP1/2 that encode BTB-ankryin transcriptional co-activators (Ha et al., 2003; 2007; Hepworth et al., 2005). Both AS1 and BOPI/2 are expressed in stage 1 leaf primordia and are excluded from the SAM by STM (Byrne et al., 2000; Norberg et al., 
2005; Jun et al., 2010). AS2 is expressed on the adaxial side of leaves, overlapping with BOP1/2 expression at the adaxial base of leaves and in the petiole (Norberg et al., 2005; Jun et al., 2010). In loss-of-function as 1, as 2, and bopl bop2 mutants, leaflets or ectopic blade growth occurs to different degrees on the petiole and $B P$ transcripts are up-regulated. Reciprocally, overexpression of $A S 1, A S 2$, or $B O P 1 / 2$ phenocopies loss-of-function $b p$ phenotypes, indicating that AS1, AS2 and BOP1/2 are negative regulators of $B P$ (Byrne et al., 2000; Ori et al., 2000; Semiarti et al., 2001; Iwakawa et al., 2002; Ha et al., 2003; 2007; 2010; Lin et al., 2003).

The mechanism for repression of $B P$ and $K N A T 2$ by AS1-AS2 was recently elucidated. AS1 and AS2 bind to the $B P$ and KNAT2 promoters directly, forming a repressor complex that contains the chromatin-remodeling protein HIRA (Xu et al., 2003; Phelps-Durr et al., 2005; Guo et al., 2008). BOP1 activates AS2 directly (Jun et al., 2010) suggesting that $B O P 1 / 2$ repression of $B P$ is indirect. Independent experiments showed that AS1, AS2 and BOP1/2 repress $B P$, KNAT2 and KNAT6 expression in leaves (Byrne et al., 2000; Ori et al., 2000; Semiarti et al., 2001; Iwakawa et al., 2002; Ha et al., 2003; 2007; Lin et al., 2003) and that leaf patterning defects in bop 1 bop 2 as 1 and bop1 bop 2 as 2 triple mutants are more severe than in bopl bop2, as 1 , or as 2 (Ha et al., 2003; 2007). However, how this phenotypic synergism correlates spatially and quantitatively with $K N O X 1$ gene misexpression in the SAM and in leaves was not known. My work was designed to address these questions.

\subsection{Materials and methods}

\subsubsection{Plant material and growth conditions}

Plant growth conditions were identical to those described in Chapter 2. Mutant alleles of as 1-1 (CS3374), as2-1 (CS3117) and axr1-3 (CS3075) were obtained from the ABRC and have been described previously (Byrne et al., 2000; Iwakawa et al., 2002; Smith and Hake, 2003; Belles- 
Boix et al., 2006; Hay et al., 2006a). Mutant alleles of $b p-1$ and $b p-2$ (introgressed into Col-0) were provided by Raju Datla (Venglat et al., 2002). The reporter lines BOPI::GUS and BOP2::GUS were previously described (McKim et al., 2008; Xu et al., 2010). KNAT2::GUS (C24 ectotype) and KNAT6::GUS (WS ectotype) were gifts from Veronique Pautot (Dockx et al., 1995; Belles-Boix et al., 2006). All mutant combinations were constructed by crossing, and where possible they were confirmed by PCR genotyping.

\subsubsection{Primers and genotyping}

Primers used for genotyping and transcript analysis are listed in Table 3.1. The strategy for genotyping bop 1-3 and bop2-1 alleles is described in Chapter 2. For genotyping bp-2, primers bp-2dCAPs-F1 and bp-2dCAPs-F2 were used to amplify a 240-bp product from genomic DNA. Only the $b p-2$ product is cleaved by MunI to yield a 211-bp fragment.

\subsubsection{Analysis of leaf initiation}

For analysis of leaf initiation, plants were grown in continuous (24h light) or long-day (16h light) photoperiods. Leaf initiation was scored by marking leaves at emergence. Leaves were counted daily until emergence of the inflorescence meristem ( $n=20$ plants).

\subsubsection{GUS staining}

Tissues were stained, embedded, and sectioned as described in Chapter 2.

\subsubsection{Quantitative RT-PCR (qPCR)}

RNA isolation, reverse transcription and qPCR were performed essentially as described in Chapter 2. Tissues were harvested from 21-day-old leaves grown in continuous light. Total cDNA was synthesized from $1 \mu \mathrm{g}$ of RNA template using Superscript III reverse transcriptase (www.invitrogen.com). qPCR was performed in triplicate using $5 \mu \mathrm{l}$ of diluted cDNA as the 
template in a reaction containing SYBR ${ }^{\circledR}$ Green I (www.sigma-aldrich.com) and IQ Supermix (www.biorad.com) using a Rotor-Gene 6000 (www.qiagen.com) thermocycler. Conditions were optimized for each primer pair and data quality was verified by melting curve analysis. Primers for $\mathrm{qPCR}$ analysis were as listed in Table 3.1. qPCR was performed with triplicates and relative transcript levels were calculated from mean of threshold cycle values Pfaffl (2001). Expression levels were normalized with values obtained using the reference gene GAPC (Hepworth et al., 2005). Experiments were repeated at least twice using independently-isolated RNA. Representative data from one biological replicate is shown.

\subsection{Results}

\subsubsection{Expression pattern of $B O P 1$ and $B O P 2$ in vegetative apices}

The double mutant bopl bop 2 has broad, leafy petioles that are similar to as 1 mutants, which have short and broad petioles, and to as 2 mutants, which form isolated leaflets near the junction of the blade and the petiole. This suggests that their functions in leaves might be similar. In situ hybridization and GUS reporter gene analysis indicate that $A S l$ is expressed in emerging leaf primordia and that $A S 2$ is expressed at the adaxial side of leaf primordia (Byrne et al., 2000; Jun et al., 2010). To confirm that the expression domain of BOPI and BOP2 overlap with $A S 1$ and $A S 2$ in shoot apices, I examined the expression patterns of $B O P 1:: G U S$ and $B O P 2:: G U S$ reporter genes in short-day grown seedlings. BOP2 was not detected in the SAM or P0 primordia but readily detected in stage 1 leaf primordia, similar to ASI (Figure 3.1a; Byrne et al., 2000). During stage 2 of leaf development, $B O P 2$ expression was restricted to the boundary between the leaf primordia and the SAM (Figure 3.1b). At stages 3 and 4 of leaf development, $B O P 2$ expression was localized to the adaxial base of leaf primordia (Figure 3.1a, b) in an overlapping pattern with AS2 (Ha et al., 2003; 2004; 2007; Hepworth et al., 2005; Norberg et al., 2005). In 
expanded leaves, BOP2 expression was detected in the petiole and mid-vein (Figure 3.1d). BOP 1::GUS was expressed in a similar pattern (not shown). These data confirm the overlapping expression domains for $B O P 1 / 2, A S 1$, and $A S 2$ in developing leaves.

To confirm whether BOP1/2 has a defect in leaf initiation similar to asl (Hay et al., 2006a), I compared the leaf initiation rate of wild-type and bopl bop 2 mutant plants in long-day and continuous light photoperiods. My data showed that the leaf initiation rate in bopl bop2 plants was consistently slower than in wild-type control plants (Figure 3.1c). These data confirm a probable role for BOP1/2 in leaf initiation, similar to AS1 (Hay et al., 2006a).

\subsubsection{BOP1/2 function redundantly with AS1/2 in promoting simple leaf form}

Similar expression patterns and loss-of-function mutant phenotypes for BOP1/2 and AS1-AS2 led me to hypothesize that BOP1/2 function redundantly with $\mathrm{AS} 1 / 2$ or that they function in a linear pathway. While Ha et al (2003) reported that leaf defects in bop1-1 as 1 and bop1-1 as2 double mutants were more severe than in single mutants, this data was considered problematic since bop1- 1 is a dominant-negative allele in the Landsberg erecta (Ler) ecotype that causes a leaf phenotype but does not cause the floral defects seen in loss-of-function bop1 bop 2 mutants (Hepworth et al., 2005). We therefore constructed the bop1 bop 2 as 1 and bop1 bop 2 as 2 triple mutants in a Col background and analyzed the resulting phenotypes. Leaves of bop1 bop2, as 1, and as 2 have growths on their petioles to different degrees (Figure 3.2b, f, g; Byrne et al., 2000; Semiarti et al., 2001; Hepworth et al., 2005; Norberg et al., 2005; Ha et al., 2007). At an early developmental stage, the leaves of bop1 bop 2 as 1 and of bop 1 bop 2 as 2 triple mutants displayed ectopic growth of tissures on the adaxial side of leaf petioles (Figure 3.2c, h). In old leaves $(>5$ weeks old) this tissue developed into leaflets, stipules, inflorescences, or flowers (Figure 3.2d, i), resembling the form of compound leaves. The growth of leaflets, inflorescence and flowers is 
never observed in bopl bop2, as1, or as 2 mutants, therefore, the genetic interaction between BOP1/2 and AS1/2 is synergistic. No synergistic defects in floral development were observed (data not shown).

Collectively these data confirm that BOP1/2 activity overlaps AS1 and AS2 in leaves of by repressing meristematic activity in the petiole region, necessary for the formation of simple leaves. These data are in agreement with Ha et al. (2007) who published a study of triple mutant phenotypes during the early stages of my project.

\subsubsection{BOP1/2 act alongside AS1 and AS2 to exclude $K N O X I$ gene expression from leaves}

Arabidopsis thaliana produces simple leaves, whereas its close relative Cardamine hirsuta produces dissected leaves with individual leaflets. These two leaf forms are the result of differential expression of KNOX1 genes during leaf development. Studies have shown that KNOX1 genes are repressed throughout Arabidopsis leaf development (Byrne, et al., 2000; Ori et al., 2000; Semiarti et al., 2001; Iwakawa., et al., 2002), but reactivated during Cardamine leaf development (Hay and Tsiantis, 2006b; Barkoulas et al., 2008). In agreement, Arabidopsis plants ectopically expressing $B P$ or $S T M$ display deeply lobed leaves with ectopic meristems in the sinus of the lobes, resembling compound leaves (Lincoln et al. 1994; Chuck et al., 1996; Gallois et al., 2002). It was unclear if the compound-leaf like phenotype in bopl bop2 as 1 and bopl bop2 as 2 triple mutants directly correlated with synergistic up-regulation of $K N O X I$ genes, though it has been shown that $K N O X 1$ genes are up-regulated in bop1 bop2, as 1 and as 2 leaves individually (Byrne, et al., 2000; Ori et al., 2000; Semiarti et al., 2001; Iwakawa., et al., 2002; Lin et al., 2003; Ha et al., 2003; 2007; Xu et al., 2003).

To test this, quantitative RT-PCR comparing KNOXI gene expression in 21-day-old leaves of WT, bop1 bop2, as 1, as2, bop1 bop2 as 1, and bopl bop2 as 2 plants was performed. 
These data showed that in mature leaves, transcripts of BP, STM, KNAT2, and KNAT6 were significantly higher in bop1 bop 2 as 1 or bop 1 bop 2 as 2 triple mutants relative to parental control plants (Student's t-test, $\mathrm{p}<0.05$ for all). This is consistent with the synergistic leaf-petiole defects in these mutants. In contrast, $P N Y$ transcripts were unchanged in all of the mutants, indicating that KNOXI genes are the main target of BOP1/2 repression in leaves (Figure 3.3). This comparison also showed that $B P$ is the main target of misexpression in all mutants.

To determine if the compound-leaf like phenotype in the triple mutants was attributable to misexpression of $B P$, we generated bopl bop2 as 1 bp and bopl bop2 as 2 bp quadruple mutants (Figure 3.2e, j). In these mutants, formation of ectopic inflorescences and flowers on leaf petioles did not occur, although leaflets still formed on the petioles. These data not only demonstrate the biological relevance of $B P$ misexpression in leaves, but also indicate that other factors must be misexpressed to generate a compound-leaf like phenotype.

Considering the growth of flowers on bop1 bop 2 as 1 and bop1 bop 2 as 2 triple mutant petioles, we reasoned that the master floral meristem regulator LEAFY (LFY) (Schultz et al., 1991; Weigel et al., 1992) may be misexpressed in triple mutant leaves. We also hypothesized that $W U S C H E L$ (WUS) may be involved, because ectopic expression of WUS results in ectopic floral development on stems (Xu et al., 2005). We examined the transcript levels of $L F Y$ and WUS in leaves of the triple mutants and their parental controls. However, we did not observe any significant change in $L F Y$ and WUS transcript levels at the 21-day-old time-point tested (data not shown).

\subsubsection{BOP1/2 represses $K N A T 2$ and $K N A T 6$ expression in shoot apices}

Quantitative RT-PCR has shown that BOP1/2 represses KNOX1 transcription in leaves (Figure 3.3), but the spatial pattern of this misexpression was unknown. To study this, I compared the 
expression patterns of $B P:: G U S, K N A T 2:: G U S$ and KNAT6::GUS in WT and bop1 bop2 leaf tissues and in the shoot apices of 25-day-old seedlings grown in short days ( $8 \mathrm{~h} \mathrm{light)} \mathrm{(Long} \mathrm{and}$ Barton, 2000).

In the apices of WT, KNAT2 was expressed in the peripheral and rib zones of the meristem and stopped at the base of young leaves (Figure 3.4a). In bop1 bop2 mutants, KNAT2 expression was intensified and expanded, with staining in the petioles of leaves and in hypocotyls (Figure 3.4a, b), overlapping with the BOPI/2 expression domain (Figure 3.1a, b, d). KNAT6 expression in WT plants was focused in the meristem-leaf boundary and in stipules (Figure 3.4c). In bop1 bop2 apices, KNAT6 expression was up-regulated in the boundary and it expanded into the petioles of emerging leaves, hypocotyls and young leaf tips, similar to KNAT2 (Figure 3.4c, d). Little or no misexpression of $B P$ was detected in bop1 bop2 shoot apices, similar to previous reports although it can be seen at the seedling stage (data not shown; Madiha Khan, unpublished data; Jun et al., 2010). However, BP is misexpressed in bop1 bop 2 midvein or to the margins of petioles in mature leaves (Madiha Khan, unpublished data), suggesting that the repression of $\mathrm{BP}$ by $\mathrm{BOP} 1 / 2$ occurs in late stages of leaf development. A potential problem with this experiment was that the reporter genes crossed into bopl bop2 were not in a homozygous state, making direct comparison with the WT control lines difficult. Nevertheless, these data support the model that BOP1/2 prevent expression of $B P$ and $K N A T 2 / 6$ at the base of leaves to shape simple leaves.

\subsection{Discussion}

The leaves of land plants differ in size and shape but are classified into two broad groups based on their degree of complexity. Simple leaves have a single flat undivided blade (no leaflet) o its petiole, while compound leaves consist of several leaflets on its petiole. The simple or compound 
leaf form is species-specific, indicating that it is under strict genetic control. Simple-compound leaf deviation has been studied extensively in Arabidopsis, Cardamine (a close relative of Arabidopsis) and tomato. Arabidopsis has simple leaves, whereas tomato and Cardamine both have dissected compound leaves (reviewed in Hay and Tsaintis, 2006b; Hay and Tsiantis, 2010).

Three pathways are known to control the simple-compound leaf deviation. The first pathway is the $K N O X 1$ gene pathway: reactivation of $K N O X 1$ gene expression during leaf development causes the formation of compound leaves (Hay and Tsiantis, 2006b). The second pathway is the auxin pathway: blocking the formation of auxin maxima in the petiole or rachis of leaves facilitates the formation of smooth petioles (Barkoulas et al., 2008). The third pathway is the CUC genes pathway: misexpression of the organ boundary genes CUC1 and CUC2 in Arabidopsis promote compound leaf development (Hasson et al., 2011).

\subsubsection{Overlapping activities of BOP1/2 and AS1-AS2 facilitate simple leaf formation}

Our data demonstrate that genetic interactions between BOP1/2 and AS1, AS2 are synergistic, since bopl bop 2 as 1 and the bopl bop 2 as 2 triple mutants display much more severe growth on their petioles than do parental control plants. This outcome was also reported by Ha et al. (2007) but in this case, quantitative and spatial analysis of $K N O X I$ genes was not performed. Moreover, Ha et al. (2007) did not report the ectopic growth of flowers on the adaxial side of mature triple mutant leaves. Our data indicate that meristematic activity in the triple mutants is limited to the leaf petiole. Our interpretation is that BOP1/2 and AS1-AS2 represent partially redundant mechanisms for promoting simple leaf shape in Arabidopsis.

\subsubsection{Turning KNOX genes off is important for simple leaf development}

KNOXI genes promote and maintain meristem activity (reviewed in Barton, 2010; Hay and Tsiantis, 2010). Our qPCR data demonstrate that BOP1/2, AS1 and AS2 function redundantly to 
keep KNOXI genes off during Arabidopsis simple leaf development. In Cardamine, which displays compound leaves, the expression domain of $S T M$ and $B P$ are expanded to the outer cell layers of P0 to P3 leaf primordia or to the leaf petiole (Hay and Tsiantis, 2006b). Knocking out STM by RNAi transforms Cardamine compound leaves into simple leaves (Hay and Tsiantis, 2006b). Our data show that mutation of $B P$ partially rescues petiole defects in bop1 bop 2 as 1 and bop1 bop2 as 2 triple mutants, demonstrating the biological relevance of $B P$ misexpression in these leaves. These data are consistent with the results of others showing that KNOXI expression domains are key determinant of leaf shape.

\subsubsection{Does BOP1/2 block the formation of auxin maxima in the petiole to inhibit leaflet}

\section{formation?}

There is a remarkable difference between the spatial regulation of auxin maxima in simple and compound leaf formation. Auxin maxima are restricted to the lamina and do not occur in the petiole in Arabidopsis (which has simple leaves). In contrast, in Cardamine (which has compound leaves) auxin maxima form on the leaf rachis, prior to leaflet outgrowth, indicating that auxin localization facilitates lateral leaflet initiation (Barkoulas et al., 2008). Polar auxin transport is mediated by a family of PIN-FORMED (PIN) auxin efflux carriers, among which PIN1 plays a key role (Hay et al., 2006a). Consistent with this, Cardamine pinl mutants have fewer leaflets on the rachis (Barkoulas et al., 2008).

Several lines of evidence suggest that leafy petioles in bopl bop 2 might correlate with auxin overload (L. Musa, 2010). First, the petioles of bop1 bop2 double mutants are broad and contain multiple parallel midveins, mimicking treatment of wild-type leaves with the auxin transport inhibitor Napthoxyacetic acid (NOA) (Sieburth, 1999). Second, the expression of an auxin-responsive reporter gene (DR5::GUS) is enhanced in bopl bop 2 leaf petioles, similar to 
the increased DR5::VENUS activity seen in the Cardamine rachis (Barkoulis et al., 2008). Third, combining bopl bop 2 and pin1En134 mutations results in very short and broad petioles, similar to the as 1 mutant and again suggestive of auxin transport defects in the petiole. The examination of DR5::VENUS activity and PIN1::GFP activity in bop1 bop2 petiole will help to elucidate if BOP1/2 regulate polar auxin transport or auxin accumulation in the petiole.

\subsubsection{Does BOP1/2 activity prevent reactivation of $C U C$ genes in the petiole to promote simple leaf formation?}

Misexpression of the organ boundary gene CUP-SHAPED COTYLEDON2 (CUC2) in Arabidopsis is a third pathway for the transformation of simple leaves into compound leaves (Hasson et al., 2011). BOP1/2 are also expressed at the boundary between the leaf meristem and the SAM (Figure 3.1b) and may directly or indirectly regulate CUC expression. It might be interesting to test whether mutation of $C U C 2$ further simplifies the structure of bop 1 bop 2 as $1 b p$ leaves and to test if $C U C$ genes are ectopically expressed in bop1 bop 2 leaves. 
Table 3.1 Primers used for genotyping and qPCR analysis.

\begin{tabular}{ll}
\hline Primer & Sequence 5'-3' \\
\hline Genotyping & \\
4H Salk RP & CGTACCCTTTGATTTTAGTATGCTG \\
4H Salk LP & GCACAATCTTTCGACTTCATCACC \\
5H Salk RP & CCCTTTTATAATCAGCATCAAGA \\
5H Salk LP & TCGACGCCGAAGTAACGAGAG \\
bp-2dCAPs-F1 & ACCCTCCTACAAGCTTACTTGGACTGCCA \\
bp-2dCAPs-R1 & GGAGGCAGAGACAGACGGTGTTGACCGCT \\
& \\
qPCR & \\
KNAT1-F1 HAY qPCR & CCATTCAGGAAGCAATGGAGTT \\
KNAT1-R1 HAY qPCR & ACTCTTCCCATCAGGATTGTTGA \\
STM-R1 HAY qPCR & GTCAAGGCCAAGATCATGGCT \\
STM -F1 HAY qPCR & TGGTGCTCCAACCTTCTGAC \\
KNAT2-F2 & TCTGAAGGACCAGCTACTACGC \\
KNAT2-R1 & ATTTTGTCGCCTTCAGTAGGGTAAG \\
KNAT6- F2 & CTTACTTCAAGCTTACATCGATTGC \\
KNAT6-R1 & CGCAGTACGTTTCCATAAATTCATC \\
BLR-F1 & TAATGTGGGTCGTGGGATTTACACC \\
BLR-R2 & ACCTCTTGTAAACCTCGTCGAGCAT \\
& \\
\hline
\end{tabular}



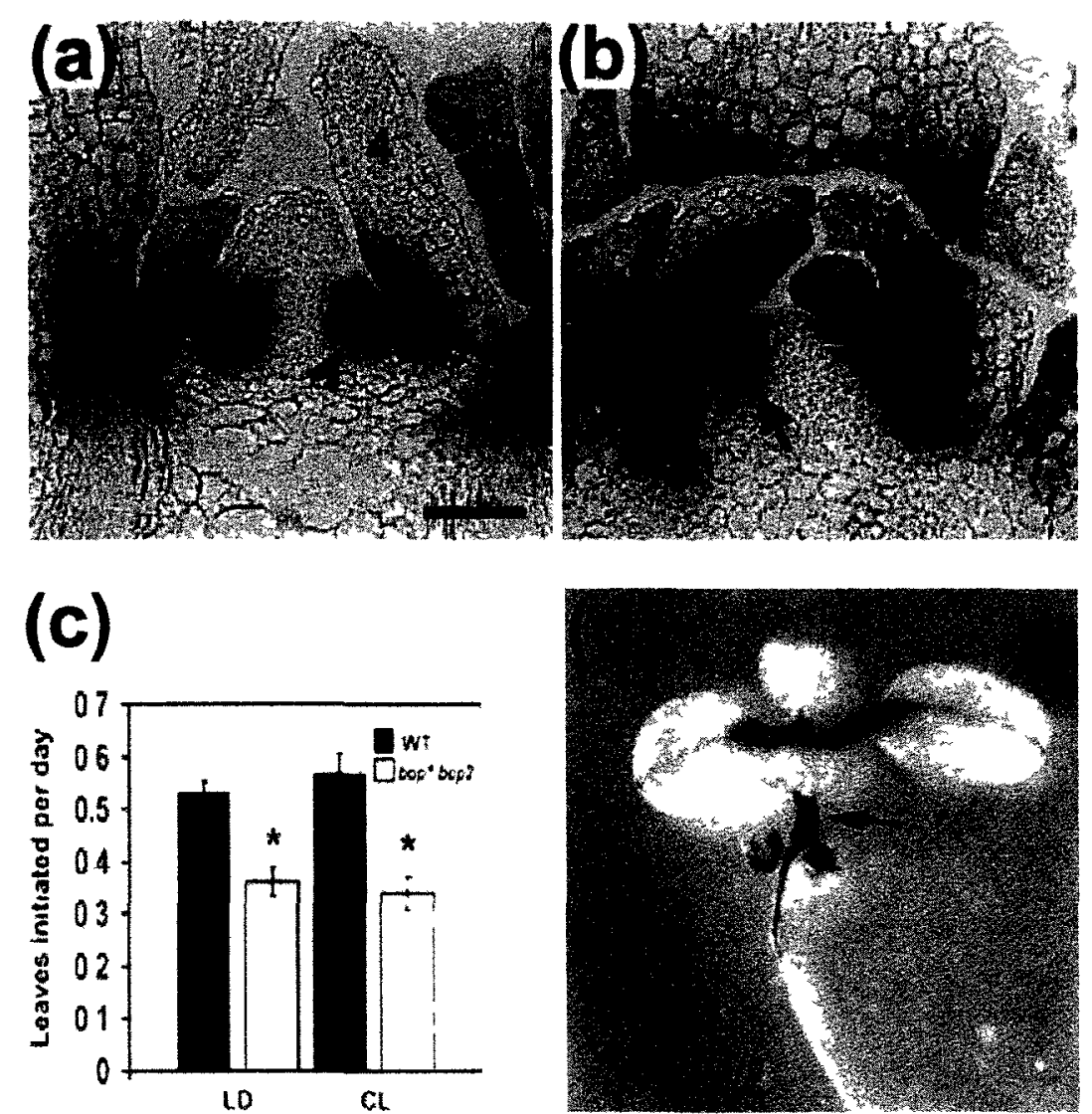

Figure 3.1 Expression pattern of BOP2::GUS in wild-type vegetative apices and leaf initiation rate in wild-type and bop1 bop2 plants.

(a-b) BOP2::GUS expression in the apices of 25-day-old wild-type seedlings grown in short days. Expression is absent from the SAM, but detected in stage 1 leaf primordia (arrowhead). At stage 2, expression localizes to the boundary between the leaf primordia and the SAM (arrow). Numbers indicate the stage of the leaf primordia. Scale bar, $50 \mu \mathrm{m}$.

(c) Lower leaf initiation rate in bop1 bop 2 than in wild-type (WT) plants under both long day (LD) and continuous light (CL) conditions. Asterisks indicate significantly differences from the wild-type (Student's t-test, $\mathrm{p}<0.05$ ). Error bars, s.e.m.

(d) BOP2::GUS expression in the WT seedlings. BOP2 is expressed in the petiole and midvein o expanded leaves and in the seedling hypocotyl (arrowhead). 

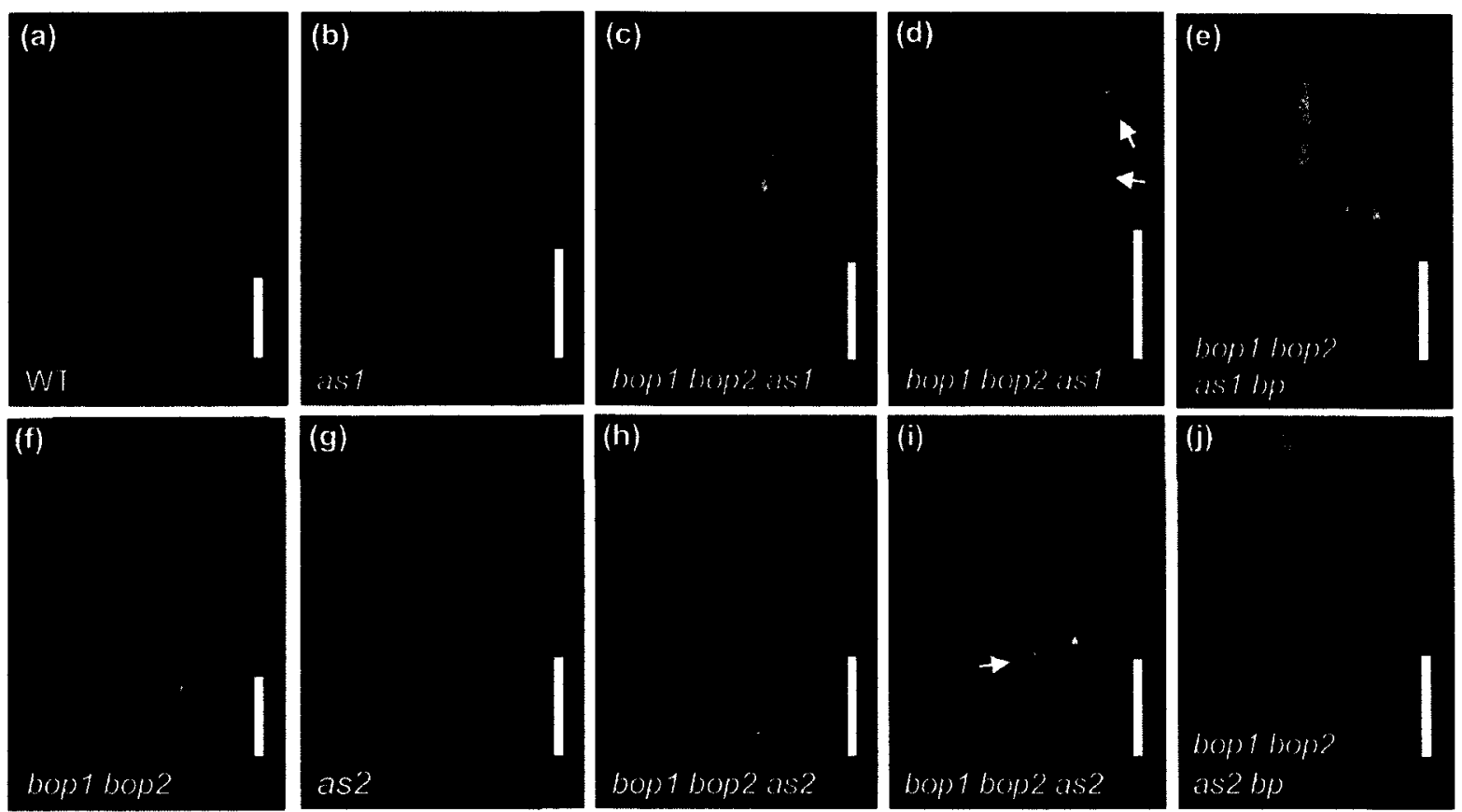

Figure 3.2 Comparison of leaf morphology in WT and mutants.

(a) Columbia wild-type.

(b) asl mutant.

(c) 3-week-old bop1 bop2 as1 mutant; enhanced meristem activity in petiole.

(d) 5-week-old bop1 bop2 as1 mutant; ectopic shoots and flowers developing from petiole (arrows).

(e) 7-week-old bop1 bop2 as 1 bp mutant; only leaflets.

(f) bop1 bop 2 mutant.

(g) as 2 mutant.

(h) 3-week-old bop1 bop 2 as 2 mutant.

(i) 5-week-old bop1 bop 2 as 2 mutant.

(j) 7-week-old bop1 bop2 as 2 bp mutant. Scale bars, $1 \mathrm{~cm}$. 


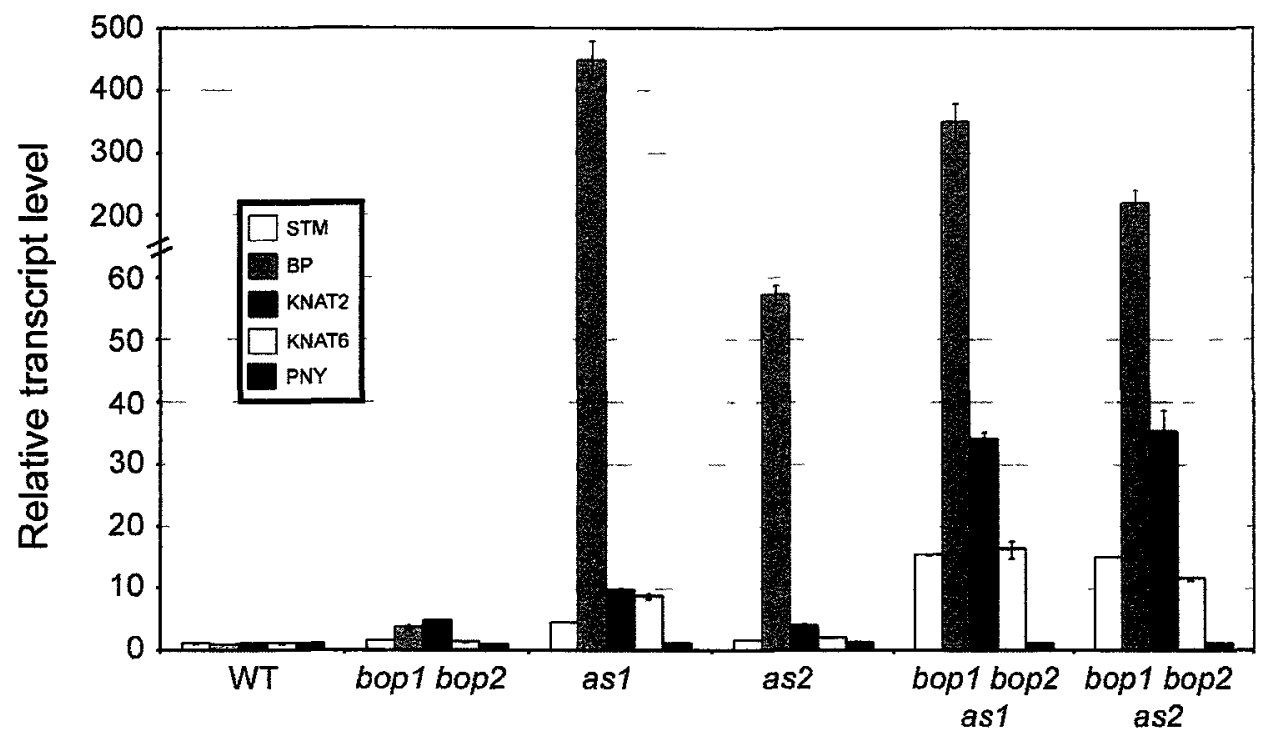

Figure 3.3 Quantitative RT-PCR analysis of class I KNOX genes and PNY in WT and mutant leaves.

Leaf tissue was collected from 21-day-old plants grown in continuous light. Relative transcript levels for $B P, K N A T 2, K N A T 6, S T M$, and $P N Y$ are shown for the indicated genotypes. Error bars, s.e.m. 


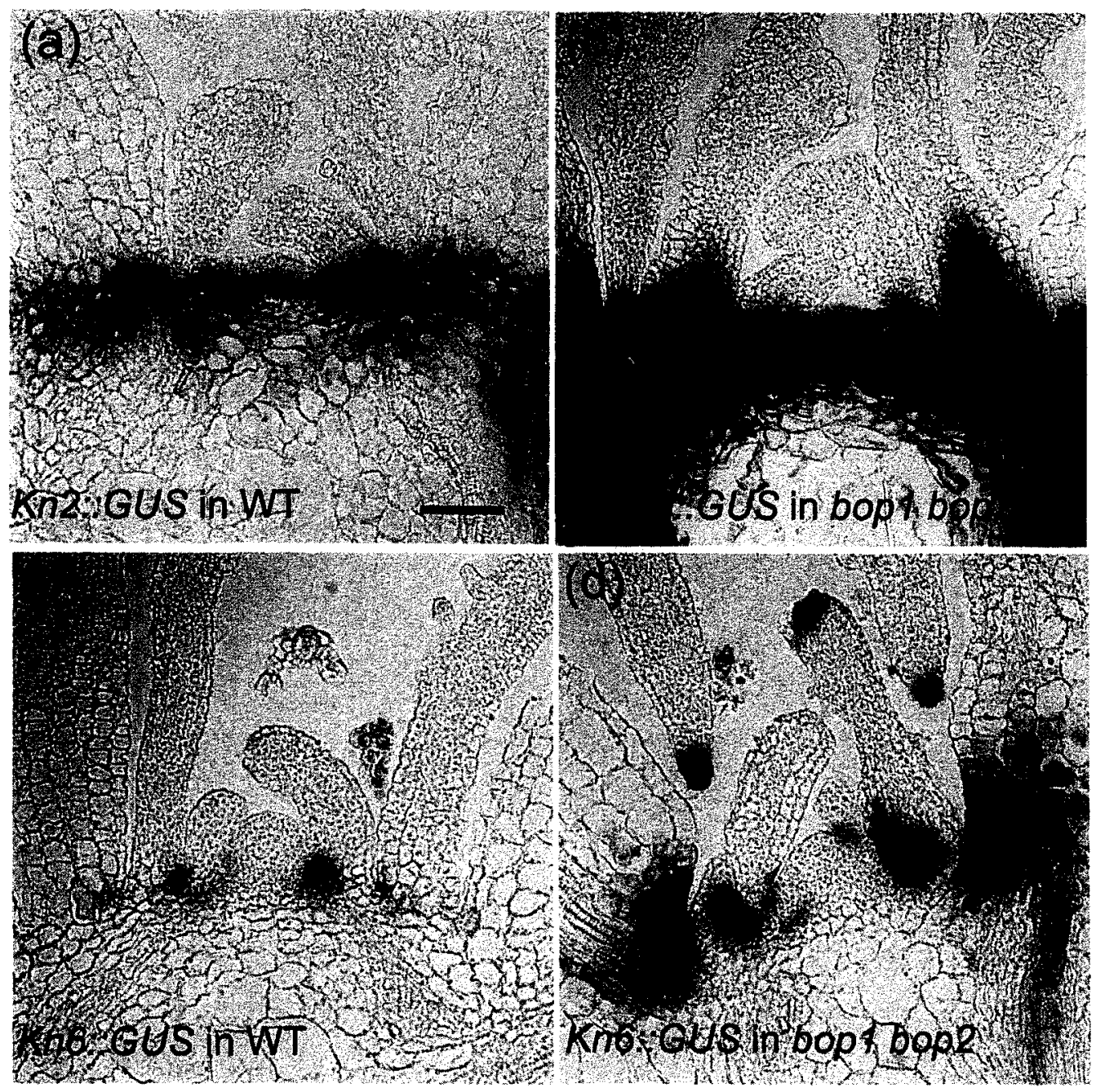

Figure 3.4 Expression patterns of KNAT2 and KNAT6 in WT and bop1 bop2 apices.

(a) KNAT2::GUS in WT. Expression is throughout the rib and peripheral zone of the SAM and at the base of leaf primordia.

(b) KNAT2::GUS in bop1 bop2. Expression is up-regulated relative to WT control apices and expands to leaf petioles and the hypocotyls.

(c) KNAT6::GUS in WT. Expression localizes at the proximal and adaxial portion of leaf primordia.

(d) KNAT6::GUS in bop1 bop2. Expression is up-regulated relative to WT control apices and misexpressed at the young leaf tips and in hypocotyls. Scale bar, $50 \mu \mathrm{m}$. 


\section{Chapter 4}

BREVIPEDICELLUS and PENNYWISE promote internode elongation in inflorescences by repressing BLADE-ON-PETIOLE1/2 and KNAT6 


\section{Abstract}

In Arabidopsis and many other plant species, the transition to flowering is tightly linked to the elongation of internodes to create the inflorescence. Internode patterning is one of the main determinants of inflorescence architecture, leading to diversity in inflorescence height and the organization of lateral structures (such as secondary shoots or flowers) on the main stem. Members of the KNOX1 (STM, BP, KNAT2 and KNAT6) and BELL (PNY, PNF, and ATH1) families of TALE homeodomain proteins share overlapping activities for the establishment and maintenance of the SAM but also control inflorescence development. Mutation of $B P$ or its interacting partner $P N Y$ dramatically alters inflorescence architecture resulting in compact internodes, clustered or downward pointing siliques, and irregular phyllotaxy. Expression analysis showed that $K N A T 2$ and $K N A T 6$ are normally expressed in the pedicel axis close to the stem in wild-type but misexpressed in the internodes and pedicels of $b p$ and $p n y$. Expression analysis on $B O P 1$ and $B O P 2$ showed that they are also expressed in the pedicel axis in wild-type, and misexpressed in the pedicels and stems of $b p$ and $p n y$. The close expression pattern between $B O P 1 / 2$ and $K N A T 2 / 6$ suggest that these factors may have a common function. In support of this, BOP1/2 gain-of-function mutants are very short with compact internodes and clustered or downward pointing siliques, similar to $b p$ or pny mutants. To investigate how $B O P 1$ and $B O P 2$ contribute to inflorescence architecture, I examined their genetic interaction with $B P$ and $P N Y$, in collaboration with Madiha Khan. My data show that BOP2 and KNAT6 expression domains are similarly enlarged in $b p$ or pny mutants, corresponding to their mutant phenotypes. My data also provide evidence that $\mathrm{BOP} 1 / 2$ are positive regulators of $K N A T 6$ and that this activation may be direct. Collectively, BP-PNY negatively regulate BOP1/2-KNAT6 in stems establishing a molecular network for the regulation of internode elongation. 


\subsection{Introduction}

Internode elongation determines a plant's height and the organization of lateral structures on the main stem and is therefore a key determinant of inflorescence architecture. During Arabidopsis vegetative development, internode elongation is suppressed and compact rosettes are produced whereas during reproductive development internode elongation is activated by KNOXI and BELL homeodomain proteins (Venglat et al., 2002; Byrne et al., 2003; Smith and Hake, 2003; Ragni et al., 2008). KNOX1 (BP, STM, KNAT2 and KNAT6) and BELL (PNY, PNF, ATH1) homeodomain proteins function redundantly during vegetative development in establishment and maintenance of the SAM (Lincoln, et al., 1994; Long et al., 1996; Byrne et al., 2002; 2003; Belles-Boix et al., 2006; Rutjens et al., 2009). However, during reproductive development, antagonistic interactions between some of these KNOXI and BELL homeodomain proteins become important for internode elongation and pedicel orientation (Smith and Hake, 2003; Smith et al., 2004; Ragni et al., 2008; Rutjens et al., 2009). Mutation of $b p$ or pny results in reduced internodes, downward pointing or clustered siliques, altered phyllotaxy, and defects in cell differentiation, albeit in slightly different patterns for each mutant (Smith and Hake, 2003; Byrne et al., 2003; Venglat et al., 2002; Douglas et al., 2002). The bp pny double mutant is extremely short (Smith and Hake, 2003) indicating overlapping activities for BP and PNY in the internodes. A second study showed that the pny pnf double mutant fails to bolt and produces only leaves (Smith et al., 2004) indicating that the activities of PNY and PNF are redundant and required for transition of the apical meristem from SAM to IM identity and for production of internodes. Yeast-2-hybrid experiments have shown that BP forms heterodimers with PNY and PNF, suggesting a mechanism for the some of the interactions seen between BP, PNY, and PNF in vivo (e.g. Kanrar et al., 2006). By contrast, loss-of-function in KNAT2 and KNAT6 or ATH1 
rescue $b p, p n y$, and/or $b p$ pny internode elongation defects indicating that the activities of BPPNY and KNAT2-KNAT6-ATH1 are antagonistic during reproductive development and required for development of the inflorescence (Ragni et al., 2008; Rutjens et al., 2009).

Several observations prompted us to test the nature of genetic interactions between BOP1/2 and BP-PNY during inflorescence development. First, strong BOP1/2 gain-of-function mutants are extremely short with downward pointing or clustered siliques (Norberg et al., 2005; Ha et al., 2007; Khan and Xu, data not shown), mimicking bp pny double mutants (Smith and Hake, 2003). Second, BOP1/2, KNAT2, and KNAT6 are co-expressed in pedicel axils where they may share a function. Third, Ragni et al. (2008) showed that knat2 knat6 mutations rescue rather than enhance $b p$ and pny mutant phenotype, revealing that the defects in these mutants are caused by misexpression of KNAT2 and KNAT6 in stems and pedicels.

We considered that gain-of-function BOP1/2 may interfere with internode elongation by activating $A S 2$, because BOP is a direct activator for $A S 2$ in leaves (Jun et al. 2010), and AS2 is a direct repressor for $B P$ (Guo et al., 2008). However, mutation of $A S 2$ only slightly restored inflorescence height and pedicel angle in $35 S:: B O P 1 / 2$ plants (Ha et al., 2007) and loss-offunction as 2 does not rescue $b p$ inflorescence defects (Byrne et al., 2002). These interactions suggested that BOP1/2 pattern internode using a mechanism that is largely independent of AS2.

I show here that $\mathrm{BP}$ and PNY restrict the expression domains of $B O P 1 / 2$ in pedicel axis. My data show that $B O P 1 / 2$ and KNAT6 are co-misexpressed in $b p$ and pny in characteristic patterns and that $\mathrm{BOP} 1 / 2$ promotes KNAT6 expression. We will find out if KNAT6 is a direct target of BOP $1 / 2$ activation in stems in the future. 


\subsection{Materials and methods}

\subsubsection{Plant material and growth conditions}

Plant growth conditions are the same as those described in Chapter 2. Mutant alleles of bop1-3, bop $2-1, b p-1$, and $b p-2$ were described in Chapter 2 and Chapter 3 . The activation-tagged overexpression line bop 1-6D was kindly provided by O. Nilsson (Norberg et al., 2005). The reporter line BOP2::GUS is described in Chapter 2 (Xu et al., 2010). All mutant combinations were constructed by crossing and confirmed by PCR genotyping where possible.

\subsubsection{Primers and genotyping}

Primers used for genotyping and transcript analysis are listed in Table 4.1. The strategy for genotyping bopl-3, bop2-1,bp-1, and $b p-2$ was as described in Chapters 2 and 3. The strategy for genotyping pny-40126, knat2-5, and knat6-1 Salk T-DNA insertion mutants was as described (www.signal.salk.edu).

\subsubsection{GUS staining}

Tissues were stained, embedded, and sectioned as before (Chapter 2).

\subsubsection{Quantitative RT-PCR (qPCR)}

RNA isolation, reverse transcription, and qPCR were performed as before (Chapters 2 and 3 ). qPCR was performed with triplicates and at least two biological replicates were repeated to ensure reproductivity. Primers used in the qPCR for this chapter were listed in Table 4.1.

\subsubsection{In situ hybridization}

In situ hybridizations were performed as before (Chapter 2). Primers used to create anti-sense probes for BP, CUC3, and KNAT6 were as listed in Table 4.1. 


\subsection{Results}

\subsubsection{BOP1/2 is misexpressed in bp and pny mutants}

Ragni et al (2008) showed that loss-of-function knat6 (and knat2 knat6) rescues bp and pny inflorescence patterning defects and that KNAT2 and KNAT6 GUS reporter genes are misexpressed in the stems and pedicels of $b p$ and pny mutants. Madiha Khan in our lab found that bopl bop 2 mutations also rescue $b p$ and pny inflorescence patterning defects, in an identical fashion to mutation of knat 2 knat6. To investigate if $B O P l / 2$ are likewise misexpressed in $b p$ or pny mutants, I examined the expression pattern of BOP2 in $b p$ and pny using a GUS reporter gene driven by the $B O P 2$ promoter (Figure 4.1 ). The $B O P 2:: G U S$ reporter line has been examined before (Xu et al., 2010; Chapter 2) and the GUS activities detected by this construct are in agreement with the BOP2 RNA presence detected by in situ hybridization (Hepworth et al., 2005; Karim et al., 2009). In wild-type (WT), BOP2 expression was detected in the floral meristem and the adaxial side of the pedicel where it joins with stem (Figure 4.1a, b, c, d). However, in $b p-2$ mutants $B O P 2$ expression was expanded to the abaxial side of pedicels and stems beneath the nodes (Figure 4.1f, g, h, i), consistent with the downward orientation of siliques in this mutant. Further longitudinal- and cross-sections of stem showed that $B O P 2$ was misexpressed in stripe cells beneath nodes in $b p-2$ mutants, which become ectopically lignified later in development (Figure 4.1g, h, i, j; Douglas et al., 2002; Venglat et al., 2002; Mele et al., 2003). In pny mutants, BOP2 expression was expanded to the stems and pedicels where siliques are clustered (Figure $4.1 \mathrm{k}, 1, \mathrm{~m}, \mathrm{n}$ ) corresponding to the pny mutant phenotype. Cross sections of the stem showed that BOP2 was misexpressed in the pny cortex and vascular bundle cells (Figure 4.10). Collectively, my data show that BOP1/2 expression domains are differentially expanded in $b p$ and pny pedicels and stems, corresponding closely to the different patterning 
defects seen in these mutants. These data indicate BP and PNY restrict BOP1/2 expression domain in the stems for proper pedicel orientation and internode elongation.

\subsubsection{KNAT6 is misexpressed in stems of bop 1-6D, bp and pny}

Overexpression of $B O P 1 / 2$ results in short plants with irregular internodes, mimicking $b p$ and pny mutants (Norberg et al., 2005; Ha et al., 2007). Ragni et al. (2008) showed that misexpression of KNAT6 is required for $b p$ and pny defects prompting me to examine the pattern of KNAT6 expression in the stems and apices of WT control plants and bop1-6D, bp, and pny mutants, in which BOP1 is differently misexpressed (bop1-6D is generated by four $35 \mathrm{~S}$ enhancers, also see Figure 4.1). The expression pattern of KNAT6 in $b p$ and pny mutants has been studied before using a KNAT6::GUS reporter gene but this reporter is not active in the shoot apex (Ragni et al., 2008) indicating that some of its control sequences are missing. To accurately determine the expression pattern of KNAT6, I employed in situ hybridization for analysis. My results showed that KNAT6 transcripts were localized at the boundary between the FM and IM in WT (Figure 4.2a), and remained at the boundary in $b p-2$ and pny apices (Figure 4.2e, i). However, it is expanded to FMs in bop $1-6 D$ apices (Figure $4.2 \mathrm{~m}$ ), similar to the BOPI/2 expression domain in WT apices (Figure2.1; Xu et al., 2010), indicating that BOP may activate KNAT6. KNAT6 transcripts were not detected in WT stems; however, they accumulated to high levels in the stems of $b o p l-6 D, b p-2$ and pny mutants. In $b p-2$, KNAT6 transcript was upregulated in the epidermal stripe cells (Figure $4.2 \mathrm{f}, \mathrm{g}, \mathrm{h}$ ), similar to the pattern of BOPI/2 misexpression in $b p-2$ mutants (Figure $4.1 \mathrm{~h}, \mathrm{i}, \mathrm{j}$ ). In pny and bop $1-6 D$ mutants, up-regulation was strongest in the vascular bundle cells (Figure $4.2 \mathrm{j}, \mathrm{k}, \mathrm{l}, \mathrm{n}, \mathrm{o}, \mathrm{p}$ ). Collectively, my data demonstrate that KNAT6 is misexpressed in bopl-6D floral meristems (corresponding to the $B O P 1 / 2$ expression domain, Figure 2.1) and in $b p$ and pny stems in a pattern that corresponds to 
the $B O P 1 / 2$ misexpression domain in these mutants. These data suggest that BOP1/2 is a positive regulator of KNAT6 expression.

\subsubsection{BOP1/2 promote $K N A T 6$ expression in the inflorescence}

To further test if BOP1/2 is a positive regulator of KNAT6 expression, I used quantitative RTPCR to examine KNAT2 and KNAT6 transcript levels in the stems and pedicels of WT control plants compared to bop1-6D, bp-2, and pny mutants. My results showed that there was no significant difference of $K N A T 2$ transcript accumulation among them (data not shown); however, the transcript levels of KNAT6 were significantly higher in bop1-6D, bp, and pny tissues compared to WT (Figure 4.3), consistent with the idea that BOP1/2 promotes KNAT6 expression. When I examined KNAT6 transcript levels in bop1 bop2 bp and bop1 bop2 pny triple mutants (they look phenotypically WT) there was a significant reduction in transcript relative $b p$ and $p n y$ control lines (Figure 4.3). This was again consistent with the idea that BOP1/2 promote KNAT6 expression. Moreover, inactivation $K N A T 6$ in $35 S:: B O P 2$ lines rescues internode elongation, confirming that up-regulation of $K N A T 6$ in $35 S:: B O P 2$ lines is biologically relevant (Madiha Khan, unpublished data).

I also examined the model that ectopic BOP $1 / 2$ restricts internode elongation by repressing $B P$. Ha et al (2007) showed by semi-quantitative RT-PCR that $B P$ transcripts are reduced in the shoots and stems of $35 S:: B O P 1 / 2$ plants. They also showed that mutation of as2 partially rescues pedicel orientation but not internode elongation in $35 S: B O P 1 / 2$ plants (Ha et al., 2007). To test if $35 S: B O P 2 / b o p 1-6 D$ phenotypes are caused by inhibition of $B P$ expression, I monitored $B P$ transcript level and distribution in WT, bopl bop2 and bopl-6D apices and stems by quantitative RT-PCR and by in situ hybridization. Surprisingly, I did not see any significant changes in $B P$ expression in response to loss or gain of BOP1/2 function (Figure $4.4 \mathrm{a}, \mathrm{b}, \mathrm{c}, \mathrm{f}$ ). To 
test if boundary cell identity at the stem-pedicel junction was intact in bop 1 bop 2 mutants, I used in situ hybridization to test for misexpression of the boundary marker $C U C 3$ in stems and pedicels but saw no differences relative to WT control plants (Figure 4.4d, e). Collectively, these data indicate that BOP1/2 exerts its effect through promotion of KNAT6 expression, and that comisexpression of $B O P 1 / 2$ and KNAT6 is required for growth restriction in stems since misexpression of KNAT6 alone does not restrict growth in the inflorescence (Dean et al., 2004).

\subsection{Discussion}

Previous studies have established that $\mathrm{BOP} 1 / 2$ is a negative regulator of $K N O X 1$ expression in leaves (Ha et al., 2003; 2007; Chapter 3). To study the genetic interaction of BOP1/2 with BP and $P N Y$, I made crosses of bop1 bop2 with pny and $b p$ mutants. The triple mutants were selected by Mahida Khan who found that $b p$ and pny did not rescue bop1 bop2 leaf defect; instead that $b p$ and pny inflorescence defects are partially or fully rescued by bop 1 bop 2 (data not shown). I worked together with Madiha Khanto figure out the mechanism for this rescue. In this Chapter, I examined the expression of $B O P I / 2, K N A T 6, B P$, and $C U C 3$ spatially and quantitatively in different genotypes. I found that $B O P 1 / 2$ and $K N A T 6$ are co-misexpressed in the stems of $b p$ and pny in similar patterns and that ectopic BOP1/2 promotes KNAT6 expression in stems. Experiments by Madiha Khan showed that growth restriction in $b p$ and $p n y$ stems requires the combined misexpression of BOP1/2 and KNAT6; the misexpression of just one of these factors does not block internode elongation.

\subsubsection{Spatial regulation of $B O P 1 / 2$ by $B P$ and $P N Y$ is required for internode elongation} Previous work has established that the KNOX1 genes STM, BP, KNAT2, and KNAT6 are expressed in the SAM and repressed in developing leaves by the overlapping activities of 
BOP1/2, AS1, and AS2 (Byrne et al., 2000; Ori et al., 2000; Semiarti et al., 2000; Ha et al., 2003; 2007; Jun et al., 2010; Chapter 3). However, in the inflorescence, BP and PNY restrict BOP1/2 and KNAT2/6 expression domains for appropriate internode elongation (Ragni et al., 2008; this study), suggesting differences in the functions of these genes during vegetative and reproductive development. Internode elongation is attributable to the proliferation and elongation of cells in the rib zone (RZ), which lies in the L3 layer of the SAM, below the central zone (CZ) (Fletcher 2002; Chapter 1). Interestingly, $B P$ is not expressed in the $\mathrm{RZ}$ of the SAM when internode elongation is suppressed, while it is expressed in the cortex of stems (corresponding to the RZ) when internode elongation is allowed during reproductive development (Lincoln et al., 1994; this study), suggesting that expression of $B P$ in the $R Z$ promotes internode elongation. Consistent with this, loss-of-function $b p$ mutants are short. Interestingly, $P N Y$ is not expressed in the RZ of vegetative SAM nor in the reproductive inflorescence meristem, nor is $B P$ expression impaired in pny mutants (Byrne et al., 2003; Smith and Hake, 2003; Smith et al., 2004). Nonetheless, pny mutants have shortened internodes between some secondary inflorescences and flowers, resulting in clusters of secondary inflorescences or flowers on the main stem. This indicates that factors other than BP are involved in internode elongation. The BELL protein ATH1 may be one of these factors. During vegetative development $A T H 1$ is expressed throughout the SAM during which time internode elongation is suppressed. Upon floral induction, $A T H 1$ is down-regulated and internode elongation begins. Consistent with this, athl mutants show ectopic internode elongation between successive rosette leaves while the $35 S:: A T H 1$ inflorescences are extremely short caused by reduced cell proliferation in the RZ (Gomez-Mena and Sablowski, 2008). Regarding their opposite expression patterns and functions, it is unclear if $B P$ represses $A T H 1$ nor if athl can rescue $b p$, but it is noteworthy that loss-of-function athl fully rescues pny 
internodes and partially rescues pny pnfflowering (Rutjins et al., 2009).

\subsubsection{Internode elongation and flowering is coordinated in Arabidopsis}

During the vegetative to reproductive transition, floral inductive signals cause the vegetative meristem to undergo morphological changes that are essential for flowering (reviewed in Liu et al., 2009a; Barton et al., 2010). Though pny and pnf single mutants have no obvious flowering time delay or morphological change of the SAM, the pny pnf double mutant fails to bolt and the size of the SAM is diminished (Smith et al., 2004). Expression and complementation studies showed that PNY and PNF function upstream of LFY and that FT requires the activities of PNY and PNF to promote internode elongation and flowering (Smith et al., 2004; Kanrar et al., 2008). Mutation in ATH1, an activator for the floral repressor FLC, can partially restore the pny pnf non-flowering defect, suggesting that PNY and PNF may repress ATH1 then FLC to coordinate flowering and internode elongation.

Studies also show that the size of the SAM is smaller in pny pnf than that of the wild-type (smith et al., 2004; Ung et al., 2011). MONOPTEROS (MP), an auxin response factor, is expressed in the $\mathrm{PZ}$ in WT inflorescence meristems and the region between $\mathrm{MP}$ expression domains indicates the CZ. In situ analysis of MP in wild type and py pnf showed that the size of the central zone between the $M P$ expression domains is reduced in pny pnf double mutant compared to that of the wild type (Ung et al., 2011), suggesting that the number of stem cells in pny pnf is reduced. Accordingly, expression of CLAVATA3 (CLV3), which is expressed at the CZ and negatively modulates the meristem size, is expanded in pny pnf and loss-of-function in CLV3 partially restored pny pnf flowering (Ung et al., 2011). Floral cells and internode cells are all generated from the activity of stem cells located in the SAM, diminished stem cells in the pny pnf indicate loss of growth potential and inability to give rise to floral meristem and $\mathrm{RZ}$ cell 
proliferation (which gives rise to internodes, Chapter 1). Taken together, these data indicate that PNY and PNF coordinate flowering and internode elongation by regulating the number of stem cells in the CZ. Loss-of-function bop1 bop 2 and knat 2 knat6 can rescue pny defects (Ragni et al., 2008; this study), but it is unknown if bop1 bop2 and knat2 knat6 can rescue the pny pnf meristem size and flowering. It will be interesting to know how BOP1/2 interact with PNY/PNF to coordinate internode elongation and flowering.

\subsubsection{BOP1/2 activate $K N A T 6$}

The qPCR analysis in stems and pedicels (Figure 4.3) showed that BOP1/2 promote KNAT6 transcript accumulation in these tissues. Interestingly, detailed expression pattern analysis showed that BOP1/2 and KNAT6 are co-misexpressed in the same tissues in $b p$ and pny, and that KNAT6 is ectopically expressed in the floral meristems of bop $1-6 D$ where $B O P 1$ is normally expressed (Figure 4.1, 4.2). Strikingly, $B P$ required for internode elongation is not significantly up- or down-regulated in bopl bop 2 or bopl- $6 \mathrm{D}$ lines suggesting that BOP1/2 exerts its effects mainly through KNAT6.

\subsubsection{Co-misexpression of $B O P I / 2$ and $K N A T 6$ is required to block internode elongation} My data show that BOP1/2 and KNAT2/6 are co-misexpressed in $b p$ and pny and that BOP1/2 promotes KNAT6 expression but at least two lines of evidence suggest that the combined activities of $\mathrm{BOP} 1 / 2$ and KNAT6 are required to restrict internode elongation. First, 35S:KNAT6 plants are not short (Dean et al., 2004). Second, bp knat2 knat6 and pny knat2 knat6 plants are phenotypically normal but presumably still misexpress BOP1/2 (Ragni et al., 2008). Third, inactivation of KNAT6 restores internode elongation in $35 \mathrm{~S}: B O P 2$ plants. Although we do not yet understand how BOP1/2-KNAT6 antagonize BP-PNY activities, it does not appear to be at the transcriptional level since $B P$ and $P N Y$ transcripts and reporter gene expressions patterns are 
normal in bop1 bop2 and bop1-6D stems (this study, Madiha Khan, unpublished data). One possibility is that BOP1/2 modifies KNAT6 function in such a way that it competes for BP-PNY DNA-binding sites to antagonistically regulate the expression of target genes that control internode elongation. Presumably, if this were the case, KNAT6 would need a BELL family partner. ATH1 is a candidate for this job since ATH1 functions antagonistically with PNY-PNF in internode elongation (Gómez-Mena and Sablowski, 2008; Rutjens et al., 2009). Alternatively, BOP1/2 proteins localize to both the cytoplasm and the nucleus (Hepworth et al. 2005; Jun et al. 2010) and may potentially influence PNY activity, which is essential for STM and possibly BP nuclear localization (Hackbush et al. 2005; Rutjens et al. 2009). Short internodes are typical of defects in GA biosynthesis (Achard and Genschik, 2009; Schwechheimer and Willige, 2009), however, analysis of GA 20-oxidase transcript levels in 35S:BOP2 and bop1-6D stems did not show a significant change (Madiha Khan, data not shown) making it uncertain if BOP1/2KNAT6 targets GA biosynthesis. Future experiments will address the mechanism of antagonism. 
Table 4.1 Primers used for genotyping, qPCR analysis, and making in situ hybridization probes

\begin{tabular}{ll}
\hline Primer & Sequence 5'-3' \\
\hline Genotyping & \\
PNY Salk_40126 RP & TTGGAATTGGAGACAAAATGTGTTA \\
PNY Salk_40126 LP & GGAACCAAGTTCAAACTCGAATCCA \\
KN2 Salk_099837 RP & CAAAAGGTGATCTCGCTGCTTTCGT \\
KN2 Salk_099837 LP & AATCTCTAGCGCAAAAGTTTTTGCT \\
KN6 Salk_054482 F2 & CTTACTTCAAGCTTACATCGATTGC \\
KN6 Salk_054482 LP & TGCTTTCTGATCACTTCAAAAGCCT \\
& \\
qPCR & \\
KNAT1-F1 HAY qPCR & CCATTCAGGAAGCAATGGAGTT \\
KNAT1-R1 HAY qPCR & ACTCTTCCATCAGGATTGTTGA \\
KNAT2-F2 & TCTGAAGGACCAGCTACTACGC \\
KNAT2-R1 & ATTTTGTCGCCTTCAGTAGGGTAAG \\
KNAT6-F2 & CTTACTTCAAGCTTACATCGATTGC \\
KNAT6-R1 & CGCAGTACGTTCCATAAATTCATC \\
In situ probes & \\
BP-LF & \\
BP-T7-RR & ATGGAAGAATACCAGCATGACAAC \\
CUC3-F1 & CATAATACGACTCACTATAGGCCTTCTCTGACTCAGAAGGATATG \\
CUC3-T7-R1 & ATGATGCTTGCGGTGGAAGATGTG \\
KNAT6-F3 (in situ) & CATAATACGACTCACTATAGGCTACAGCTGGAATCCTAAAGGACATGG \\
KNAT6-T7-R1 & ATGGATGGAATGTACAATTCCATTC \\
& CATAATACGACTCACTATAGGTCATTCCTCGGTAAAGAATGAT \\
\hline
\end{tabular}


Figure 4.1 Expression patterns of $B O P 2$ in wild-type, $b p-2$ and pny plants.

(a) to (d) wild-type control; BOP2::GUS localized in the adaxial pedicel stem cells.

(e) Cross section of WT stem; BOP2::GUS expression was not detected.

(f) to (i) $b p-2$; ectopic expression in the abaxial pedicel cells and stem cells.

(j) Cross section of $b p-2$ stem; ectopic expression in the stripe cells under the node.

(k) to (n) pny; ectopic expression in the stem and pedicel cells where internode elongation is irregular.

(o) Cross section of pny stem; ectopic expression in the cortex and vascular bundle cells. Scale bars, $100 \mu \mathrm{m}$. 


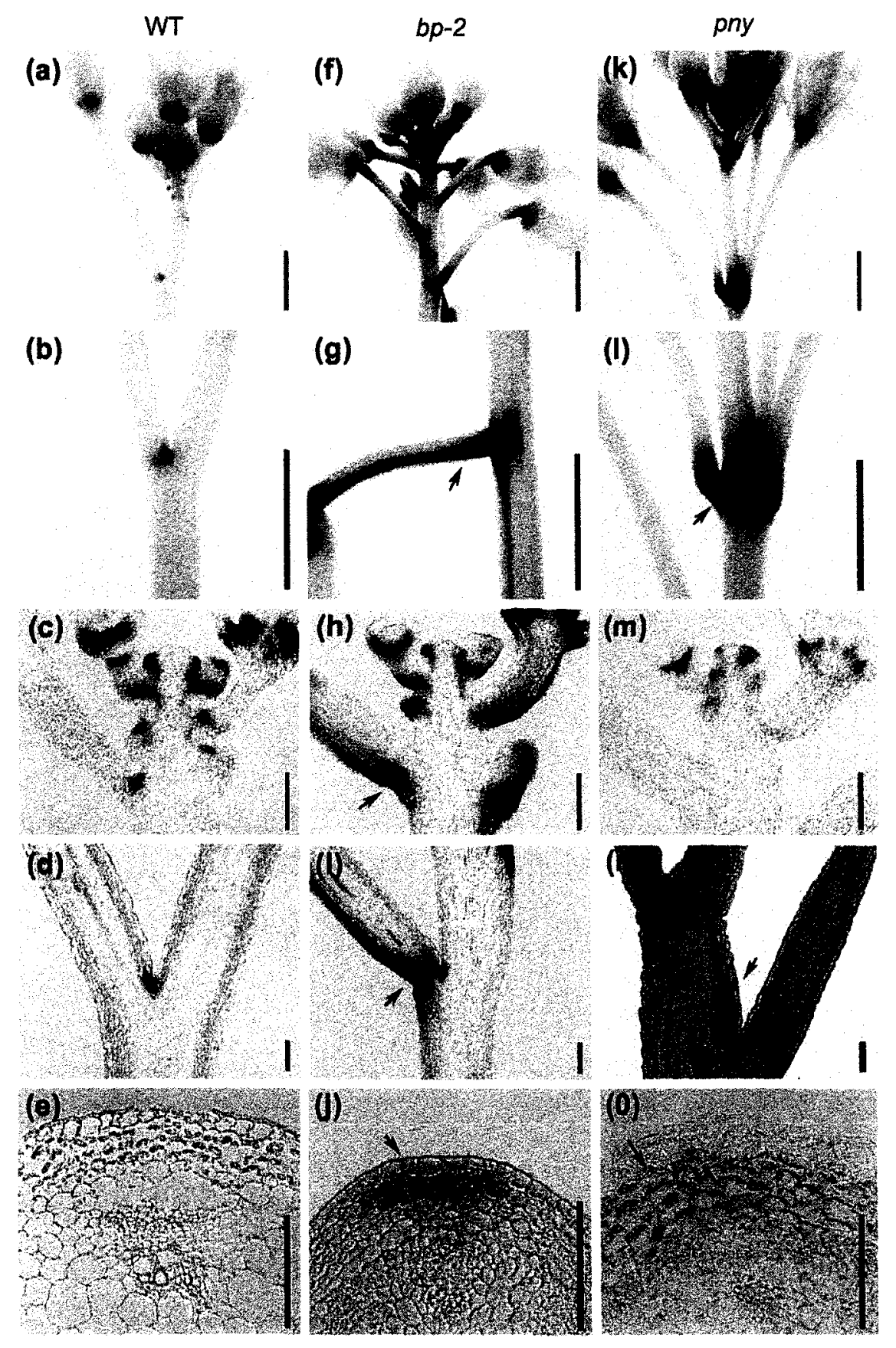




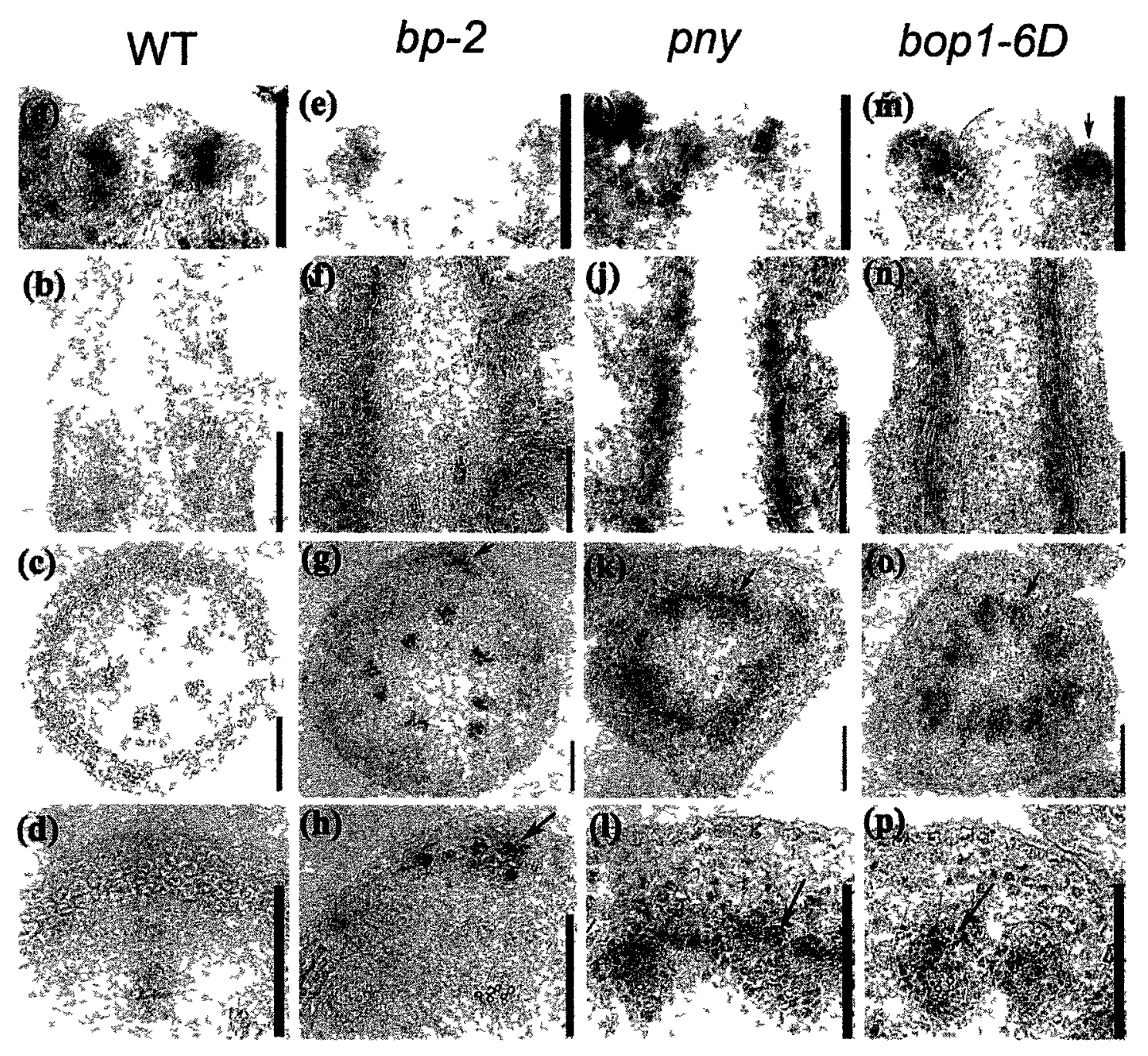

Figure 4.2 In situ analysis of KNAT6 transcript accumulation in WT and mutant apices and stems.

(a-d) wild-type control; transcript localizes at the boundary between floral primordia and the inflorescence meristem.

(e-h) $b p-2$; ectopic expression in the epidermal cells and stripe cells.

(i-l) pny; ectopic expression in the vascular bundle cells.

(m-p) bop 1-6D; ectopic expression in the vascular bundle cells. Scale bars, $100 \mu \mathrm{m}$. 


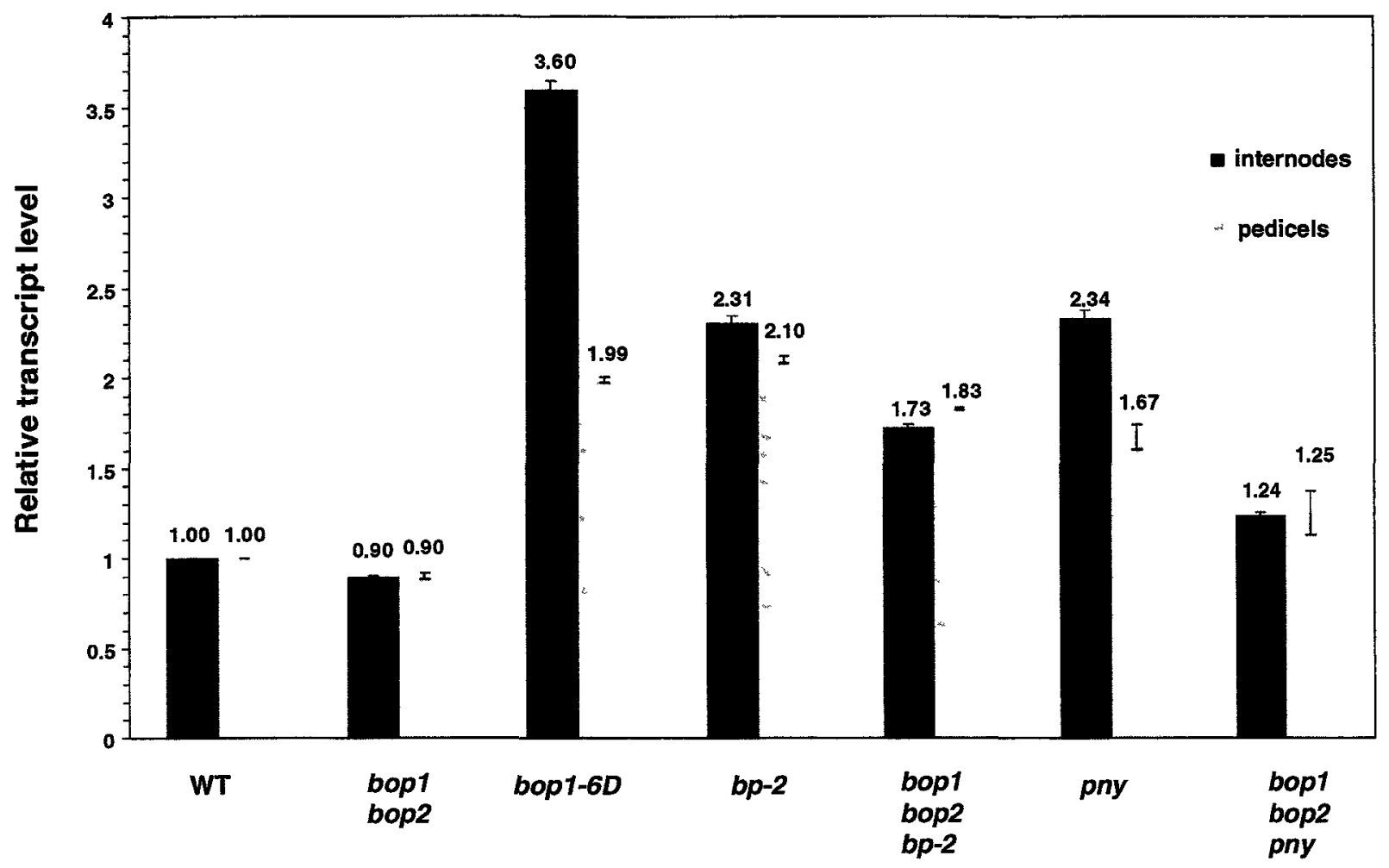

Figure 4.3 Quantitative analysis of KNAT6 transcript accumulation in WT and mutant internodes and pedicels.

RNAs were isolated from internodes or pedicels and analyzed by qPCR for KNAT6 expression. Genotypes are as indicated. KNAT6 transcript level is high in bop1-6D, bp-2 and pny, tissues compared to WT, and lower in bopl bop 2 bp-2 and bopl bop 2 pny tissues compared to bp-2 and pny, respectively. Values shown are mean. Error bars indicate s.e.m. 


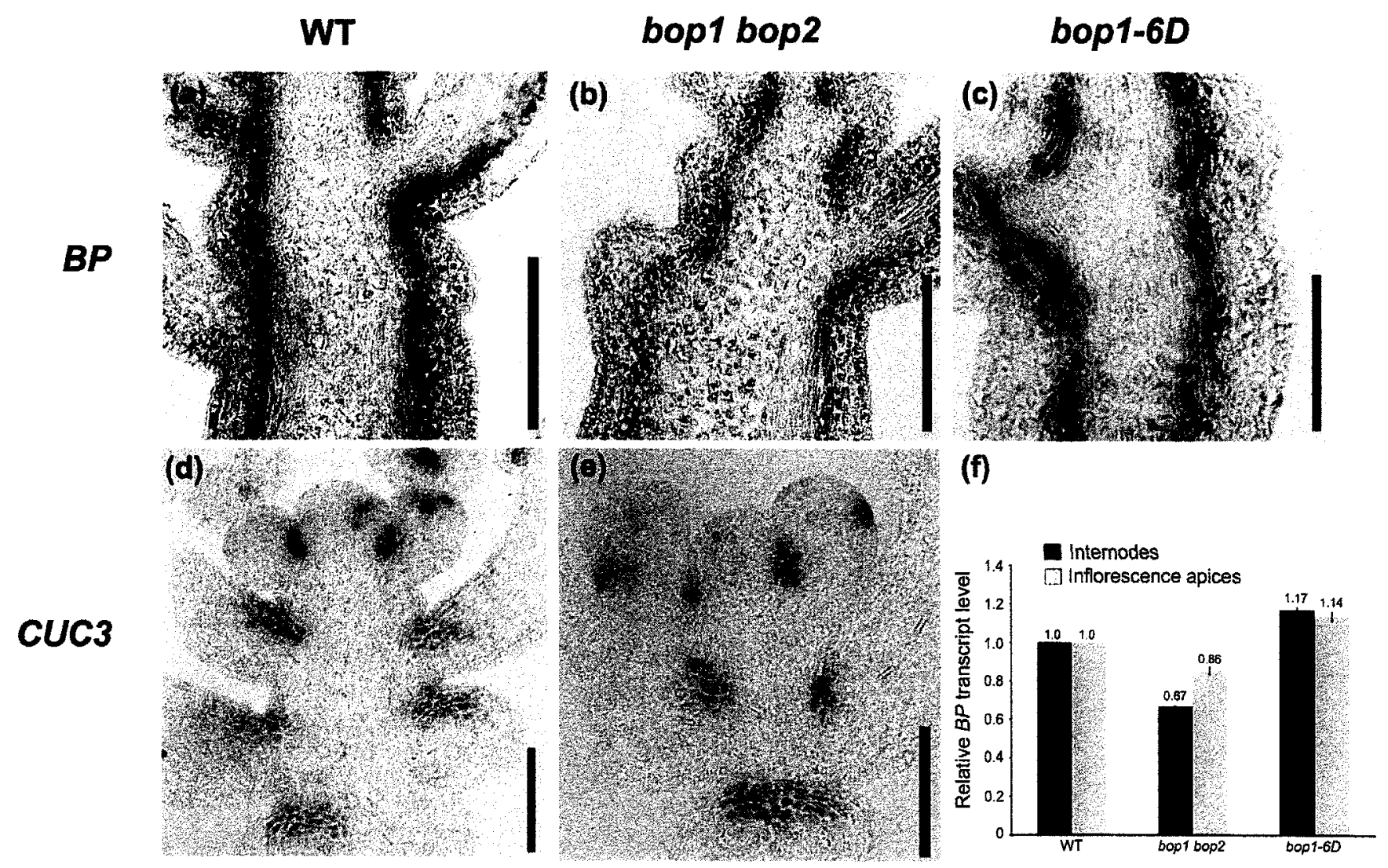

Figure 4.4 Spatial and quantitative analysis of $B P$ and $C U C 3$ in different genotypes.

(a) to (c) In situ hybridization analysis BP transcript accumulation in stems of:

(a) wild-type control; high levels of $B P$ transcript accumulation in stems and pedicels.

(b) bopl bop2.

(c) bop1-6D. No significant change of $B P$ transcript accumulation was detected.

(d) and (e) In situ hybridization analysis CUC3 transcript accumulation in apices of:

(d) wild-type control and;

(e) bop1 bop2. CUC3 transcripts are accumulated at the boundaries of organs. The expression pattern was the same in wild-type as in bop1 bop2. Scale bars, $100 \mu \mathrm{m}$.

(f) Quantitative RT-PCR analysis of relative $B P$ transcript levels in the internodes and apices. $B P$ transcript levels are similar for all genotypes. Values shown are mean. Error bars indicate s.e.m. 


\section{Chapter 5}

Summary and future directions 


\subsection{Summary}

This thesis examined the roles of the BLADE-ON-PETIOLE (BOP1) and BOP2 genes in regulating Arabidopsis flower, leaf, and inflorescence architecture. I will summarize my findings and give directions for future studies in this Chapter.

My studies in the model plant species Arabidopsis thaliana have provided insight into the role that BOP1/2 plays in controlling floral meristem identity, leaf determinacy, and internode length.

In floral meristems, I show that BOP1 and BOP2 function redundantly with LFY, a master regulator of floral-meristem identity, to promote the expression of $A P 1$, a key marker of commitment to floral fate. All three activities then converge in the floral meristem during stages 2-3 to down-regulate the expression of inflorescence identity genes including $A G L 24, S O C 1$, and FUL to ensure the floral determinacy. After stage 3, BOP1/2 function redundantly with LFY and AP1 to promote A-class floral patterning.

In leaves, I show that BOP1 and BOP2 in the petiole function redundantly with the leaf identity factors AS1 and AS2 to maintain the stable repression of $K N O X 1$ meristematic genes to facilitate the formation of a simple leaf.

In inflorescences, I show that expression domains of boundary genes BOP1/2 and KNAT6 are confined to pedicel axils by the overlapping activities of the KNOX1 family member BP and its interacting BELL partner PNY. My data show that BOP2 and KNAT6 expression domains are differentially enlarged in $b p$ and pny mutants, corresponding to the distinctive patterns of short internodes, clustered or downward-oriented siliques, and defects in cell differentiation typical of 
these mutants. My data further suggest that $\mathrm{BOP} 1 / 2$ are positive regulators of $K N A T 6$ expression and that this regulation may be direct.

Collectively, this work illustrates how modulation of meristematic activity and cell fate by $\mathrm{BOP} 1 / 2$ dramatically alters plant architecture. It will be interesting to determine whether BOP1/2 activity or expression patterns vary between plants species and form a platform for creating architectural diversity.

\subsection{Future directions}

\subsubsection{What contributes to the blade growth on the petiole?}

Blade growth on the petiole is a major defect in bopl bop 2 mutants. Ha and coworkers (2007; 2010) interpret that the blade-on-petiole phenotype is caused by the combined misexpression of $K N O X I$ and $Y A B$ abaxial identity genes and provide genetic evidence in support of this model. Our interpretation of the phenotype is that it reflects a partial conversion from simple to compound leaves. It will be very interesting to further investigate this simple to compound leaf transformation hypothesis by looking at genes that control simple-compound leaf deviation in WT and bopl bop2.

\subsubsection{Regulation of $K N O X 1$ genes in seedlings}

I have shown in Chapter 3 that KNAT2 and KNAT6 are up-regulated in the SAM of bop1 bop2 (Figure 3). However, the KNAT2::GUS and KNAT6::GUS lines that were used in this experiment were not homozygous lines. To confirm that KNAT2 and KNAT6 are up regulated in the SAM of bop1 bop 2 mutants, qPCR and in situ hybridization should be performed to re-examine KNAT2 and KNAT6 expression. 


\subsubsection{Examine polar auxin transport and auxin localization in the petiole}

Local auxin maxima are present on the Cardamine rachis, and inhibition of polar auxin transport on the rachis can reduce the complexity of leaflets on Cadarmine petioles (Barkoulas et al., 2008), indicating that local auxin maxima facilitated by polar auxin transport triggers organ (including leaflet) formation. Lama Musa from our lab has shown that the broad petiole of bop1 bop2 resembles the phenotype of plants treated with the auxin influx inhibitor NOA. Therefore, it is very likely that $\mathrm{BOP} 1 / 2$ regulate auxin abundance and that mistransport of auxin in the petiole triggers the formation of blade-on-petiole. To investigate this, DR5::VENUS and PIN1::GFP lines could be used to study their localization in WT and bop1 bop2 in petioles.

\subsubsection{Examine $C U C$ exp ression and genetic interaction of $B O P 1 / 2$ with $C U C$ genes}

Although $B O P 1 / 2$ are expressed at the boundary between the leaf primordia and the SAM in stage 2 leaves (Figure $3.1 \mathrm{~b}$ ) their role at the boundary is not clear. Recently it was shown that misexpression of the organ boundary gene CUP-SHAPED COTYLEDON2 (CUC2) in Arabidopsis causes transformation of simple leaves into compound leaves (Hasson et al., 2011). Based on the fact that both $B O P 1 / 2$ and $C U C$ are expressed at the boundary and both play roles in simple leaf formation, we hypothesized that there may be some interaction between them.

bopl bop 2 cuc1 and bop1 bop 2 cuc 3 triple mutants have been constructed and these triple mutants do not show enhanced organ separation phenotypes. Since there is redundancy between CUC genes (CUC1, CUC2, and CUC3), bopl bop2 quadruple mutants with $c u c$ genes will be constructed to confirm their mode of interaction. $C U C$ and BOP $1 / 2$ genes are both required for KNOXI repression in leaves (this study; Hasson et al., 2011) suggesting that BOP1/2 and CUC genes may function in separate or converging pathways to repress $K N O X 1$ genes. The phenotype of the quadruple mutants would tell if they function synergistically or not. To examine if BOP1/2 
function upstream of CUC genes, the expression pattern of $\mathrm{CUC1} / 2 / 3$ could be examined in wild-type and bopl bop2. If there is misexpression or down regulation of CUC genes in bop1 bop2 mutant, it suggests that $B O P 1 / 2$ function upstream of $C U C$ genes. Similarly, the expression of $B O P 1 / 2$ in $c u c$ double mutants would tell us if $C U C$ genes function upstream of $B O P 1 / 2$.

\subsubsection{Bract formation}

My work in Chapter 2 has suggested that BOP1/2 are required to repress bract outgrowth in collaboration with LFY and AGL24. Liu et al (2009b) showed that the AGL24 in conjunction with $S O C 1$ and $S V P$ suppress bract formation. However, the network regulating bract formation is not clear, which could be potentially interesting to study. The expression domains of $L F Y$, $B O P 1 / 2, A G L 24, S O C 1$ and $S V P$ define three distinct zones in late stage 2 floral primordia. $L F Y$, $B O P 1 / 2$ and $S V P$ share the floral meristem zone but partition differently. $L F Y$ is expressed in the adaxial part of the floral meristem with $B O P 1 / 2$ and $S V P$ expression marking the boundary between the floral meristem and the cryptic bract zones (Weigel et al., 1992; Hartmann et al., 2000; Karim et al., 2009; Xu et al., 2010). AGL24 and SOCl are only expressed in the cryptic bract zone (Yu et al., 2004; Xu et al., 2010); It should be investigated whether the activities from the floral meristem zone, cryptic bract zone and the boundary converge to facilitate growth of the flower and suppression of the bract.

The following triple or quadruple mutants bop1 bop2 svp, bop1 bop2 soc1, bop1 bop2 agl24 svp, bop1 bop2 agl24 socl, bop1 bop2 svp soc1, lfy svp agl24, and bop bop2 lfy agl24 could be generated see if floral development is suppressed and bract development is enhanced in

these triple or quadruple mutants. RNA accumulation of STM in the stage 2 floral primordia of these triple or quadruple mutants would be examined to see if the meristematic cells have 
changed. Also the cell division activity would be examined in the floral meristem zone and bract zone using the cell division marker histone $\mathrm{H} 4$ to see if the pattern of cell division has changed in the stage 2 floral primordia.

\subsubsection{Direct target of BOP1/2 in the stem}

My data suggested that KNAT6 is a major transcriptional target of BOP $1 / 2$ in stems; however, we need to find out if KNAT6 is a direct target of BOP1/2. To test this, we can make use of protein fusions to the rat glutacorticoid receptor (GR) and the yeast-one-hybrid system together with BiFC.

\subsubsection{Identification of direct targets of BOP1 by 35S::BOP1-GR}

The introduction of a GR fusion protein into plants in combination with application of the steroid hormone dexamethasone (Dex) and cycloheximide (CHX) is an essential tool for identifying direct target genes in plants (Wagner et al., 1999; Yu et al., 2004; Jun et al. 2010). Dex is an inducer for the nuclear localization of the fusion protein and $\mathrm{CHX}$ is a protein synthesis inhibitor, which inhibits the synthesis of proteins allowing the identification of a direct target (Sablowski and Meyerowitz, 1998). If the transcript levels of a target gene show that is activated or repressed shortly after Dex treatment, and this activation or repression remains constant with CHX treatment, then the gene is likely a direct target of the GR fusion protein. I have constructed a $35 S:: B O P 1-G R$ fusion gene and transformed it into plants. We will treat the transgenic plants with Dex and harvest tissues $1 \mathrm{~h}, 4 \mathrm{~h}$, and $24 \mathrm{~h}$ after Dex treatment to see if KNAT6 is up-regulated linearly. We will also treat the plants with Mock, Dex, CHX, and Dex + CHX regimes to see if $K N A T 6$ expression levels remain the same in Dex and Dex + CHX treated tissues. 


\subsubsection{Identification of the genomic region of the target that BOP-TGA complex binds}

The fact that BOP $1 / 2$ proteins do not contain a DNA binding domain, they associates with TGA transcription factors in yeast (Hepworth et al., 2005; Jun et al., 2010; Xu et al., 2010) and that BOP1 binds to its direct targets $\mathrm{AS} 1$ and $\mathrm{AP} 1$ at sites that TGA transcription factors potentially recognize and bind leads us to hypothesis that BOP1/2 may be recruited to the promoters of the genes it regulates through association with TGA factors. BOP1/2 activate KNAT6 (Chapter4), and this activation could be direct. In addition, the KNAT6 regulatory region contains potential TGA binding sites (http://www.athamap.de/), prompting us to examine if BOP1/2 function together with any of the TGA transcription factors to directly regulate KNAT6 expression. The yeast-one-hybrid system could be employed to study if any of the ten TGA factors in Arabidopsis binds directly to the KNAT6 promoter. If one or more TGAs bind to the KNAT6 promoter, further deletion series on the KNAT6 promoter could be created to test where the TGAs bind. Further, the BiFC analysis could be performed to study if the TGAs associate with BOP1/2 in plant cells. Alternatively, chromatin immunoprecipitation (ChIP) assay could be employed to examine the BOP-TGA binding sites on the regulatory region of the target gene (here refers to KNAT6). 


\section{References}

Abe, M., Kobayashi, Y., Yamamoto S., Daimon, Y., Yamaguchi, A., Ikeda, Y., Ichinoki, H., Notaguchi, M., Goto, K., and Araki, T. (2005) FD, a bZIP protein mediating signals from the floral pathway integrator FT at the shoot apex. Science 309, 1052-1056.

Achard, P., and Genschik, P. (2009) Releasing the brakes of plant growth: how GAs shutdown DELLA proteins. J. Exp. Bot. 60, 1085-1092.

Aida, M., and Tasaka, M. (2006a) Morphogenesis and patterning at the organ boundaries in the higher plant shoot apex. Plant Mol. Biol. 60, 915-928.

Aida, M., and Tasaka, M. (2006b) Genetic control of shoot organ boundaries. Curr. Opin. Plant Biol. 9, 72-77.

Aida, M., Ishida, T., and Tasaka, M. (1999) Shoot apical meristem and cotyledon formation during Arabidopsis embryogenesis: interaction among the CUP-SHAPED COTYLEDON and SHOOT MERISTEMLESS genes. Development 126, 1563-1570.

Aida, M., Ishida, T., Fukaki, H., Fujisawa, H., and Tasaka, M. (1997) Genes involved in organ separation in Arabidopsis: an analysis of the cup-shaped cotyledon mutant. Plant Cell 9, 841-857.

Aida, M., Vernoux, T., Furutani, M., Traas, J., and Tasaka M. (2002) Roles of PINFORMED1 and MONOPTEROS in pattern formation of the apical region of the Arabidopsis embryo. Development 129, 3965-3974.

Avery L., and Wasserman, S. (1992) Ordering gene function: the interpretation of epistasis in regulatory hierarchies. Trends Genet. 8, 312-316.

Bao, X., Franks, R.G., Levin, J.Z., and Liu, Z. (2004) Repression of AGAMOUS by BELLRINGER in floral and inflorescence meristems. Plant Cell 16, 1478-1489.

Barkoulas, M., Hay, A., Kougioumoutzi, E., and Tsiantis, M. (2008) A developmental framework for dissected leaf formation in the Arabidopsis relative Cardamine hirsuta. Nat. Genet. 40, 1136-1141.

Barton, M.K. (2010) Twenty years on: the inner workings of the shoot apical meristem: a developmental dynamo. Dev. Biol. 341, 95-113.

Bellaoui, M., Pidkowich, M.S., Samach, A., Kushalappa, K., Kohalmi, S.E., Modrusan, Z., Crosby, W.L., and Haughn, G.W. (2001) The Arabidopsis BELL1 and KNOX TALE homeodomain proteins interact through a domain conserved between plants and animals. Plant Cell 13, 2455-2470.

Belles-Boix, E., Hamant, O., Witiak, S. M., Morin, H., Traas, J., and Pautot, V. (2006) KNAT6: an Arabidopsis homeobox gene involved in meristem activity and organ separation. Plant Cell 18, 1900-1907.

Benková, E., Michneiwicz, M., Suaer, M., Teichmann, T., Seifertova, D., Jürgens, G., and Friml, J. (2003) Local efflux-dependent auxin gradients as a common module for plant organ formation. Cell 115, 591-602. 
Blázquez, M.A., Ferrándiz, C., Madueno, F., and Parcy, F. (2006) How floral meristems are built. Plant Mol. Biol. 60, 855-870.

Blázquez, M.A., Soowal, L.N., Lee, I., and Weigel, D. (1997) LFY expression and flower initiation in Arabidopsis. Development 124, 3835-3844.

Borghi, L., Bureau, M., and Simon, R. (2007) Arabidopsis JAGGED LATERAL ORGANS is expressed in boundaries and coordinates KNOX and PIN activity. Plant Cell 19, 1795-1808.

Bowman, J.L., and Eshed, Y. (2000) Formation and maintenance of the shoot apical meristem. Trends Plant Sci. 5, 110-115.

Bowman, J.L., Alvarez, J., Weigel, D., Meyerowitz, E.M., and Smyth, D.R. (1993) Control of flower development in Arabidopsis thaliana by APETALA1 and interacting genes. Development 119, 721-743.

Bowman, J.L., Smyth, D.R., and Meyerowitz, E.M. (1989) Genes directing flower development in Arabidopsis. Plant Cell 1, 37-52.

Bowman, J.L., Smyth, D.R., and Meyerowitz, E.M. (1991) Genetic interactions among floral homeotic genes of Arabidopsis. Development 112, 1-20.

Boyle, P., Su, E.L., Rochon, A., Shearer, H.L., Murmu, J., Chu, J.Y., Fobert, P.R., and Després, C. (2009) The BTB/POZ domain of the Arabidopsis disease resistance protein NPR1 interacts with the repression domain of TGA2 to negate its function. Plant Cell 21, 3700-3713.

Brand, U., Fletcher, J.C., Hobe, M., Meyerowitz, E.M., and Simon, R. (2000) Dependence of stem cell fate in Arabidopsis on a feedback loop regulated by CLV3 activity. Science 289, $617-619$.

Breuil-Broyer, S., Morel, P., de Almeida-Engler, J., Coustham, V., Negrutiu, I. and Trehin, C. (2004) High-resolution boundary analysis during Arabidopsis thaliana flower development. Plant J. 38, 182-192.

Busch, M.A., Bomblies, K., and Weigel, D. (1999) Activation of a floral homeotic gene in Arabidopsis. Science 285, 585-587.

Byrne, M.E. (2006) Shoot meristem function and leaf polarity: the role of Class III HD- $\square$ ZIP genes. PLoS Genet 2, e89.

Byrne, M.E., Barley, R., Curtis, M., Arroyo, J.M., Dunham, M., Hudson, A., and Martienssen, R.A. (2000) ASYMMETRIC LEAVES1 mediates leaf patterning and stem cell function in Arabidopsis. Nature 408, 967-971.

Byrne, M.E., Groover, A.T., Fontana, J.R., and Martienssen, R.A. (2003) Phyllotactic pattern and stem cell fate are determined by the Arabidopsis homeobox gene BELLRINGER. Development 130, 3941-3950.

Byrne, M.E., Simorowski, J., and Martienssen, R.A. (2002) ASYMMETRIC LEAVESI reveals knox gene redundancy in Arabidopsis. Development 129, 1957-1965.

Cao, H., Li, X., and Dong, X. (1998) Generation of broad-spectrum disease resistance by overexpression of an essential regulatory gene in systemic acquired resistance. Proc. Natl. Acad. Sci. USA 95, 6531-6536. 
Chae, E., Tan, Q.K., Hill, T.A., and Irish, V.F. (2008) An Arabidopsis F-box protein acts as a transcriptional co-factor to regulate floral development. Development 135, 1235-1245.

Chen, X. (2004) A microRNA as a translational repressor of APETALA2 in Arabidopsis flower development. Science 303, 2022-2025.

Chuck, G., Lincoln, C., and Hake, S. (1996) KNATl induces lobed leaves with ectopic meristems when overexpressed in Arabidopsis. Plant Cell 8, 1277-1289.

Citovsky, V., Lee, L.Y., Vyas, S., Glick, E., Chen, M.H., Vainstein, A., Gafni, Y., Gelvin, S.B., and Tzfira, T. (2006) Subcellular localization of interacting proteins by bimolecular fluorescence complementation in planta. $J$. Mol. Biol. 362, 1120-31.

Clark, S.E., Running, M.P., and Meyerowitz, E.M. (1993) CLAVATA1, a regulator of meristem and flower development in Arabidopsis. Development 119, 397-418.

Clark, S.E., Running, M.P., and Meyerowitz, E.M. (1996) CLAVATA3 is a specific regulator of shoot and floral meristem development affecting the same processes as CLAVATA1. Development 121, 2057-2067.

Clough, C.F., and Bent, A.F. (1998) Floral dip: a simplified method for Agrobacteriummediated transformation of Arabidopsis thaliana. Plant $J$. 16, 735-743.

Coen, E., and Meyerowitz, E.M. (1991) The war of the whorls: genetic interactions controlling flower development. Nature 353, 31-37.

Cole, M., Nolte, C., and Werr, W. (2006) Nuclear import of the transcription factor SHOOT MERISTEMLESS depends on heterodimerization with BLH proteins expressed in discrete sub-domains of the shoot apical meristem of Arabidopsis thaliana. Nucl. Acids Res. 34, 12811292.

Das, P., Ito, T., Wellmer, F., Vernoux, T., Dedieu, A., Traas, J., and Meyerowitz, E.M. (2009) Floral stem cell termination involves the direct regulation of AGAMOUS by PERIANTHIA. Development 136, 1605-1611.

Dean, G., Casson, S., and Lindsey, K. (2004) KNAT6 gene of Arabidopsis is expressed in roots and is required for correct lateral root formation. Plant Mol. Biol. 54, 71-84.

Deshaies, R.J. (1999) SCF and Cullin/RING H2-based ubiquitin ligases. Annu. Rev. Cell Dev. Biol. 15, 435-467.

Després, C., DeLong, C., Glaze, S., Liu, E., and Fobert, P.R. (2000) The Arabidopsis NPR1/NIM1 protein enhances the DNA binding activity of a subgroup of the TGA family of bZIP transcription factors. Plant Cell 12, 279-290.

Dinneny, J.R., Yadegari, R., Fischer, R. R., Yanofsky, M.F., and Weigel, D. (2004) The role of JAGGED in shaping lateral organs. Development 131, 1101-1110.

Ditta, G., Pinyopich, A., Robles, P., Pelaz, S., and Yanofsky, M. F. (2004) The SEP4 Gene of Arabidopsis thaliana Functions in Floral Organ and Meristem Identity. Curr. Biol. 14, $1935-$ 1940.

Dong, X. (2004) NPR1, all things considered. Curr. Opin. Plant Biol. 7, 547-552.

Douglas, S.J., Chuck, G., Dengler, R.E., Pelecanda, L., and Riggs, C.D. (2002) KNAT1 and ERECTA regulate inflorescence architecture in Arabidopsis. Plant Cell 14, 547-558. 
Drews, G.N., Bowman, J.L., and Meyerowitz, E.M. (1991) Negative regulation of the Arabidopsis homeotic gene AGAMOUS by the APETALA2 product. Cell 65, 991-1002.

Emery, J.F., Floyd, S.K., Alvarez, J., Eshed, Y., Hawker, N.P., Ishaki, A., Baum, S.F., and Bowman, J.L. (2003) Radial patterning of Arabidopsis shoots by class III HD-ZIP and KANADI genes. Curr. Biol. 13, 1768-1774.

Eshed, Y., Izhaki, A., Baum, S. F., Floyd, S. K., and Bowman, J. L. (2004) Asymmetric leaf development and blade expansion in Arabidopsis are mediated by KANADI and YABBY activities. Development 131, 2997-3006.

Ferrándiz, C., Gu, Q., Martienssen, R., and Yanofsky, M.F. (2000) Redundant regulation of meristem-identity and plant architecture by FRUITFULL, APETALA1, and CAULIFLOWER. Development 127, 725-734.

Fletcher, J.C. (2002) Shoot and floral meristem maintenance in Arabidopsis. Annu. Rev. Plant Biol. 53, 45-66.

Fletcher, J.C., Brand, U., Running, M.P., Simon, R., and Meyerowitz, E.M. (1999) Signaling of cell fate decisions by CLAVATA3 in Arabidopsis shoot meristems. Science 283, 19111914.

Furutani, M., Vernoux, T., Traas, J., Kato, T., Tasaka, M., and Aida, M. (2004) PINFORMED1 and PINOID regulate boundary formation and cotyledon development in Arabidopsis embryos. Development 131, 5021-5030.

Gallois, J.L., Woodward, C., Reddy, G.V., and Sablowski, R. (2002) Combined SHOOT MERISTEMLESS and WUSCHEL trigger ectopic organogenesis in Arabidopsis. Development 129, 3207-3217.

Galuschka, C., Schindler, M., Bülow, L., and Hehl, R. (2007) AthaMap web tools for the analysis and identification of co-regulated genes. Nucl. Acids. Res. 35, D857-D862.

Gälweiler, L., Guan, C., Müller, A., Wisman E., Mendgen, K., Yephremov, A., and Palme, K. (1998) Regulation of polar auxin transport by AtPIN1 in Arabidopsis vascular tissue. Science 282, 2226-2230.

Gomez-Mena, C., and Sablowski, R. (2008) ARABIDOPSIS THALIANA HOMEOBOX GENE1 Establishes the Basal Boundaries of Shoot Organs and Controls Stem Growth. Plant Cell 20, 2059-2072.

Goto, K., and Meyerowitz, E.M. (1994) Function and regulation of the Arabidopsis floral homeotic gene PISTILLATA. Genes Dev. 8, 1548-1560.

Gregis, V., Sessa, A., Colombo, L., and Kater, M.M. (2008) AGAMOUS-LIKE24 and SHORT VEGETATIVE PHASE determine floral meristem identity in Arabidopsis. Plant $J$. 56, 891-902.

Guo, M., Thomas, J., Collins, G., and Timmermans, M.C. (2008) Direct repression of KNOX loci by the ASYMMETRIC LEAVES1 complex of Arabidopsis. Plant Cell 20, 48-58.

Ha, C.M., Jun, J.H., Nam, H.G., and Fletcher, J.C. (2007) BLADE-ON-PETIOLE1 and 2 control Arabidopsis lateral organ fate through regulation of LOB domain and adaxial-abaxial polarity genes. Plant Cell 19, 1809-1825. 
Ha, C.M., Jun, J.H., and Fletcher, J.C. (2010) Control of Arabidopsis leaf morphogenesis through regulation of the YABBY and KNOX families of transcription factors. Genetics 186, 197-206.

Ha, C.M., Jun, J.H., Nam, H.G., and Fletcher, J.C. (2004) BLADE-ON-PETIOLE1 encodes a $\mathrm{BTB} / \mathrm{POZ}$ domain protein required for leaf morphogenesis in Arabidopsis thaliana. Plant Cell Physiol. 45, 1361-1370.

Ha, C.M., Kim, G.T., Kim, B.C., Jun, J.H., Soh, M.S., Ueno, Y., Machida, Y., Tsukaya, H., and Nam, H.G. (2003) The BLADE-ON-PETIOLE1 gene controls leaf pattern formation through the modulation of meristematic activity in Arabidopsis. Development 130, 161-172.

Hackbusch, J., Richter, K., Muller, J., Salamini F., and Uhrig, J.F. (2005) A central role of Arabidopsis thaliana ovate family proteins in networking and subcellular localization of 3aaloop extension homedomain proteins. Proc. Natl. Acad. Sci. USA 102, 4908-4912.

Hamant, O., and Pauvot, V. (2010) Plant development: a TALE story. C. R. Biol. 333, 371-381.

Hartmann, U., Höhmann, S., Nettesheim, K., Wisman, E., Saedler, H., and Huijser, P. (2000) Molecular cloning of SVP: a negative regulator of the floral transition in Arabidopsis. Plant J. 21, 351-360.

Hasson, A., Blein, T., and Laufs, P. (2010) Leaving the meristem behind: The genetic and molecular control of leaf patterning and morphogenesis. C.R. Biol. 333, 350-360.

Hasson, A., Plessis, A., Blein, T., Adroher, B., Grigg, S., Tsiantis, M., Boudaoud, A., Damerval, C. and Laufs, P. (2011) Evolution and diverse roles of the CUP-SHAPED COTYLEDON genes in Arabidopsis leaf development. Plant Cell 23, 54-68.

Haughn, G.W., and Somerville, C.R. (1988) Genetic control of morphogenesis in Arabidopsis. Dev. Genet. 9, 73-89.

Hay, A., and Tsiantis, M. (2006b) The genetic basis for differences in leaf form between Arabidopsis thaliana and its wild relative Cardamine hirsuta. Nat. Genet. 38, 942-947.

Hay, A., and Tsiantis, M. (2010) KNOX genes: versatile regulators of plant development and diversity. Development 137, 3153-3165.

Hay, A., Barkoulas, M., and Tsiantis, M. (2006a) ASYMMETRIC LEAVES1 and auxin activities converge to repress BREVIPEDICELLUS expression and promote leaf development in Arabidopsis. Development 133, 3955-3961.

Hempel, F.D., Weigel, D., Mandel, M.A., Ditta, G., Zambryski, P., Feldman, L.J., and Yanofsky, M.F. (1997) Floral determination and expression of floral regulatory genes in Arabidopsis. Development 124, 3845-3853.

Hepworth, S.R., Klenz, J.E., and Haughn, G.W. (2006) UFO in the Arabidopsis inflorescence apex is required for floral-meristem identity and bract suppression. Planta 223, 769-778.

Hepworth, S.R., Valverde, F., Ravenscroft, D., Mouradov, A., and Coupland, G. (2002) Antagonistic regulation of flowering-time gene SOC 1 by CONSTANS and FLC via separate promoter motifs. EMBO J. 21, 4327-4337. 
Hepworth, S.R., Zhang, Y., McKim, S., Li, X., and Haughn, G.W. (2005) BLADE-ONPETIOLE-dependent signaling controls leaf and floral patterning in Arabidopsis. Plant Cell 17, 1434-1448.

Hibara, K. i., Karim, M., Takada, S., Taoka, K. i., Furutani, M., Aida, M., and Tasaka, M. (2006) Arabidopsis CUP-SHAPED COTYLEDON3 regulates postembryonic shoot meristem and organ boundary formation. Plant Cell 18, 2946-2957.

Hofer, J., Turner, L., Hellens, R., Ambrose, M., Matthews, P., Michael, A., and Ellis, N. (1997) UNIFOLIATA regulates leaf and flower morphogenesis in pea. Curr. Biol. 7, 581587.

Honma,T., and Goto, K. (2001) Complexes of MADS-box proteins are sufficient to convert leaves into floral organs. Nature 409, 525-529.

Huala, E., and Sussex, I.M. (1992) LEAFY interacts with floral homeotic genes to regulate Arabidopsis floral development. Plant Cell 4, 901-913.

Husbands, A., Bell, E.M., Shuai, B., Smith, H.M.S., and Springer, P. S. (2007) LATERAL ORGAN BOUNDARIES defines a new family of DNA-binding transcription factors and can interact with specific bHLH proteins. Nucl. Acid. Res. 35, 6663-6671.

Immink, R.G., Tonaco, I.A., de Folter, S., Shchennikova, A. Van Dijk, A.D., BusscherLange, J., Borst, J.W., and Angenent, G.C. (2009) SEPALLATA3: the 'glue' for MADS box transcription factor complex formation. Genome Biol. 25, R24.

Irish, V.F., and Sussex, I.M. (1990) Function of the apetalal gene during Arabidopsis floral development. Plant Cell 2, 741-753.

Iwakawa, H., Ueno, Y., Semiarti, E., Onouchi, H., Kojima, S., Tsukaya, H., Hasebe, M., Soma, T., Ikezaki, M., Machida, C., and Machida, Y. (2002) The ASYMMETRIC LEAVES2 gene of Arabidopsis thaliana, required for formation of a symmetric flat leaf lamina, encodes a member of a novel family of proteins characterized by cysteine repeats and a leucine zipper. Plant Cell Physiol. 43, 467-478.

Jack, T. (2004) Molecular and genetic mechanisms of floral control. Plant Cell 16, S1-S17.

Jasinski, S., Piazza, P., Craft, J., Hay, A., Woolley, L., Rieu, I., Phillips, A., Hedden, P., and Tsiantis, M. (2005) KNOX action in Arabidopsis is mediated by coordinate regulation of cytokinin and gibberellin activities. Curr. Biol. 15, 1560-1565.

Jun, J.H., Ha, C.M., and Fletcher, J.C. (2010) BLADE-ON-PETIOLE1 coordinates organ determinancy and axial polarity in Arabidopsis by directly activating ASYMMETRIC LEAVES2. Plant Cell 22, 62-76.

Kanrar, S., Bhattacharya, M., Arthur, B., Courtier, J., and Smith, H.M.S. (2008) Regulatory networks that function to specify flower meristems require the function of homeobox genes PENNYWISE and POUND-FOOLISH in Arabidopsis. Plant J. 54, 924937.

Kanrar, S., Onguka, O., and Smith, H.M. (2006) Arabidopsis inflorescence architecture requires the activities of KNOX-BELL homeodomain heterodimers. Planta 224, 1163-1173. 
Karim, M.R., Hirota, A., Kwaitkowska, D., Tasaka, M., and Aida, M. (2009) A role for Arabidopsis PUCHI in floral meristem identity and bract suppression. Plant Cell 21, 13601372.

Kayes, J.M., and Clark, S.E. (1998) CLAVATA2, a regulator of meristem and organ development in Arabidopsis. Development 125, 3843-3851.

Kempin, S.A., Savidge, B., and Yanofsky, M.F. (1995) Molecular basis of the cauliflower phenotype in Arabidopsis. Science 267, 522-525.

Kerstetter, R.A., Bollman, K., Taylor, R.A., Bomblies, K., and Poethig, R.S. (2001) KANADI regulates organ polarity in Arabidopsis. Nature 411, 706-709.

Kinkema, M., Fan, W., and Dong, X. (2000) Nuclear localization of NPR1 is required for activation of PR gene expression. Plant Cell 12, 2339-2350.

Kobayashi, Y., and Weigel, D. (2007) Move on up, it's time for change-mobile signals controlling photoperiod-dependent flowering. Genes Dev. 21, 2371-2384.

Kohalmi, S.E., Reader, L.J.W., Samach, A., Nowak, J., Haughn, G.W. and Crosby, W.L. (1998) Identification and characterization of protein interactions using the yeast 2-hybrid system. In Plant Molecular Biology Manual M1, S.B. Gelvin and R.A. Schilperoort, eds (Dordrecht, The Netherlands: Kluwer Academic Publishers), pp. 1-30.

Krizek, B., and Fletcher, J. (2005) Molecular mechanisms of flower development: an armchair guide. Nat. Rev. Genet. 6, 668-698.

Lamb, R.S., Hill, T.A., Tan, Q.K., and Irish, V.F. (2002) Regulation of APETALA3 floral homeotic gene expression by meristem identity genes. Development 129, 2079-2086.

Laux, T., Mayer, K.F., Berger, J., and Jurgens, G. (1996) The WUSCHEL gene is required for shoot and floral meristem integrity in Arabidopsis. Development 122, 87-96.

Lee, D.K., Geisler, M., and Springer, P.S. (2009) LATERAL ORGAN FUSION1 and LATERAL ORGAN FUSION2 function in lateral organ separation and axillary meristem formation in Arabidopsis. Development 136, 2423-2432.

Lee, J., Oh, M., Park, H., and Lee, I. (2008) SOC1 translocated to the nucleus by interaction with AGL24 directly regulates LEAFY. Plant J. 55, 832-843.

Lenhard, M., Bohnert, A., Jürgens, G., and Laux T. (2001) Termination of stem cell maintenance in Arabidopsis floral meristems by interactions between WUSCHEL and AGAMOUS. Cell 105, 805-814.

Levin, J.Z., and Meyerowitz, E.M. (1995) UFO: an Arabidopsis gene involved in both floral meristem and floral organ development. Plant Cell 7, 529-548.

Lewis, M.W., Leslie, M.E., and Liljegren, S.J. (2006) Plant separation: 50 ways to leave your mother. Curr. Opin. Plant Biol. 9, 59-65.

Leyser, H.M., Lincoln, C.A., Timpte, C., Lammer, D., Turner, J., and Estelle, M. (1993) Arabidopsis auxin-resistance gene AXR1 encodes a protein related to ubiquitin-activating enzyme E1. Nature 364, 161-164.

Leyser, O., Lincoln, C.A., Timpte, C., Lammer, D., Turner, J., and Estelle, M. (1993) Arabidopsis auxin-resistance gene AXR1 encodes a protein related to ubiquitin-activating 
enzyme E1. Nature 364, 161-164.

Li, S., Lauri, A., Ziemann, M., Busch, A., Bhave, M., and Zachgo, S. (2009) Nuclear activity of ROXY1, a glutaredoxin interacting with TGA factors, is required for petal development in Arabidopsis thaliana. Plant Cell 21, 429-441.

Liljegren, S.J., and Yanofsky, M.F. (1996) Genetic control of shoot and flower meristem behavior. Curr. Opin. Cell Biol. 8, 865-869.

Liljegren, S.J., Gustafson-Brown, C., Pinyopich, A., Ditta, G.S., and Yanofsky, M.F. (1999) Interactions among APETALA1, LEAFY, and TERMINAL FLOWER1 specify meristem fate. Plant Cell 11, 10007-1018.

Lin, W., Shuai, B., and Springer, P. (2003) The Arabidopsis LATERAL ORGAN $B O U N D A R I E S$-domain gene ASYMMETRIC LEAVES2 functions in the repression of KNOX gene expression and in adaxial-abaxial patterning. Plant Cell 15, 2241-2252.

Lincoln, C., Long, J., Yamaguchi, J., Serikawa, K. and Hake, S. (1994) A Knotted1-like homeobox gene in Arabidopsis is expressed in the vegetative meristem and dramatically alters leaf morphology when overexpressed intransgenic plants. Plant Cell 6, 1859-1876.

Liu C., Chen, H., Er, H.L., Soo, M.H., Kumar, P.P., Han, J.-H., Liou, Y.C. and Yu, H. (2008) Direct interaction of AGL24 and SOC1 integrates flowering signals in Arabidopsis. Development 135, 1481-1491.

Liu, C., Thong, Z. and Yu, H. (2009a) Coming into bloom: the specification of floral meristems. Development 136, 3379-3391.

Liu, C., Xi, W., Shen, L., Tan, C. and Yu, H. (2009b) Regulation of floral patterning by flowering time genes. Dev. Cell 16, 711-722.

Liu, C., Zhou, J., Bracha-Drori, K., Yalvosky, S., Ito, T. and Yu, H. (2007) Specification of Arabidopsis floral meristem identity by repression of flowering time genes. Development 134, 1901-1910.

Liu, Z., and Meyerowitz, E.M. (1995) LEUNIG regulates AGAMOUS expression in Arabidopsis flowers. Development 121, 975-991.

Lohmann, J.U., and Weigel, D. (2002) Building beauty: the genetic control of floral patterning. Dev. Cell 2, 135-142.

Lohmann, J.U., Hong, R.L., Hobe, M., Busch, M.A., Parcy, F., Simon, R., and Weigel, D. (2001) A molecular link between stem cell regulation and floral patterning in Arabidopsis. Cell 105, 793-803.

Long, J., and Barton, M. K. (2000) Initiation of axillary and floral meristems in Arabidopsis. Dev. Biol. 218, 341-353.

Long, J.A., and Barton, M.K. (1998) The development of apical embryonic pattern in Arabidopsis. Development 125, 3027-3035.

Long, J.A., Moan, E.I., Medford, J.I., and Barton, M.K. (1996) A member of the KNOTTED class of homeodomain proteins encoded by the STM gene of Arabidopsis. Nature 379, 66-69.

Maier, A.T., Stehling-Sun, S., Wollmann, H., Demar, M., Hong, R.L., Haubeiß, S., Weigel, D., and Lohmann, J.U. (2009) Dual roles of the bZIP transcription factor PERIANTHIA in 
the control of floral architecture and homeotic gene expression. Development 136, 16131620.

Maier, A.T., Stehling-Sun, S., Wollmann, H., Demar, M., Hong, R.L., Haubeib, S., Weigel, D., and Lohmann, J.U. (2009) Dual roles of the bZIP transcription factor PERIANTHIA in the control of floral architecture and homeotic gene expression. Development 136, 16131620 .

Mandel, A.M., Gustafson-Brown, C., Savidge, B., and Yanofsky, M.F. (1992) Molecular characterization of the Arabidopsis floral homeotic gene APETALA1. Nature 360, 273-277.

Mandel, M.A., and Yanofsky, M. (1995) The Arabidopsis AGL8 MADS box gene is expressed in inflorescence meristems and is negatively regulated by APETALA1. Plant Cell 7, 17631771.

Mayer, K.F.X., Schoof, H., Haeker, A., Lenhard, M., Jürgens, G., and Laux, T. (1998) Role of WUSCHEL in regulating stem cell fate in the Arabidopsis shoot meristem. Cell 95, 805815.

McKim, S., Stenvik, G.E., Butenko, M.A., Dristiansen, W., Cho, S.K., Hepworth, S.R., Aalen, R.B., and Haughn, G.W. (2008) The BLADE-ON-PETIOLE genes are essential for abscission zone formation in Arabidopsis. Development 135, 1537-1546.

McKim, S.M. (2009) Roles of the BLADE-ON-PETIOLE genes in Arabidopsis thaliana lateral organ development. Ph.D. thesis. University of British Columbia.

McSteen, P, and Leyser, O. (2005) Shoot branching. Annu. Rev. Plant Biol. 56, 353-374.

Mele, G., Ori, N., Sato, Y., and Hake, S. (2003) The knotted1-like homeobox gene BREVIPEDICELLUS regulates cell differentiation by modulating metabolic pathways. Genes \& Development 17, 2088-2093.

Meyerowitz, E.M. (1987) Arabidopsis thaliana. Annu Rev Genet. 21, 93-111.

Michaels, S.D. (2009) Flowering time regulation produces much fruit. Curr. Opin. Plant Biol. 12, 75-80.

Michaels, S.D., Ditta, G., Gustafson-Brown, C., Pelaz, S., Yanofsky, M., and Amasino, R.M. (2003) AGL24 acts as a promoter of flowering in Arabidopsis and is positively regulated by vernalization. Plant J. 33, 867-874.

Mou, Z., Fan, W., and Dong, X. (2003) Inducers of plant systemic acquired resistance regulate NPR1 function through redox changes. Cell 113, 934-944.

Musa, L. (2011) Analysis of BLADE-ON-PETIOLE interactions with auxin in control of organ initiation and leaf patterning in Arabidopsis thaliana. M.Sc. thesis, Carleton University.

Norberg, M., Holmlund, M., and Nilsson, O. (2005) The BLADE-ON-PETIOLE genes act redundantly to control growth and development of lateral organs. Development 132, 22032213.

Ohno, C.K., Reddy, G.V., Heisler, M.G.B., and Meyerowitz, E.M. (2004) The Arabidopsis JAGGED gene encodes a zinc finger protein that promotes leaf tissue development. Development 131, 1111-1122. 
Okada, K., Ueda, J., Komaki, M., and Bell, C.J. (1991) Requirement of the auxin polar transport system in the early stages of Arabidopsis floral bud formation. Plant Cell 3, 677684.

Ori, N., Eshed, Y., Chuck, G., Bowman, J.L., and Hake, S. (2000) Mechanisms that control knox gene expression in the Arabidopsis shoot. Development 127, 5523-5532.

Pape, S., Thurow, C. and Gatz, C. (2010) The Arabidopsis PR-1 promoter contains multiple integration sites for the coactivator NPR1 and the repressor SNI1. Plant Phsyiol. 154, 18051818.

Parcy, F. (2005) Flowering: a time for integration. Int. J. Dev. Biol. 49, 585-593.

Parcy, F., Nilsson, O., Busch, M.A., Lee, I., and Weigel, D. (1998) A genetic framework for floral patterning. Nature 395, 561-566.

Pautot, V., Dockx, J., Hamant, O., Kronenberger, J., Grandjean, O., Jublot, D., and Traas, J. (2001) KNAT2: evidence for a link between knotted-like genes and carpel development. Plant Cell 13, 1719-1734.

Pelaz, S., Gustafson-Brown, C., Kohalmi, S.E., Crosby, W.L., and Yanofsky, M.F. (2001a) APETALA1 and SEPALLATA3 interact to promote flower development. Plant J. 26, 38594.

Pelaz, S., Tapia-Lopez, R., Alvarez-Buylla, E.R., and Yanofsky, M.F. (2001b) Conversion of leaves into petals in Arabidopsis. Curr. Biol. 11, 182-184.

Pelaz, Z., Ditta, S., Baumann, E., Wisman, E., and Yanofsky, M.F. (2000) B and C floral organ identity functions require SEPALLATA MADS-box genes. Nature 405, 200-203.

Pérez-Pérez, J.M., Candela, H., and Micol, J.L. (2009) Understanding synergy in genetic interactions. Trends Genet. 25, 368-376.

Pfaffl, M.W. (2001) A new mathematical model for relative quantification in real-time RT-PCR. Nucl. Acids. Res. 29, e45.

Phelps-Durr, T.L., Thomas, J., Vahab, P., and Timmermans, M.C.P (2005) Maize rough sheath2 and its Arabidopsis orthologue ASYMMETRIC LEAVES1 interact with HIRA, a predicted histone chaperone, to maintain knox gene silencing and determinacy during organogenesis. Plant Cell 17, 2886-2898.

Pickett, F.B. and Meeks-Wagner, D.R. (1995) Seeing double: appreciating genetic redundancy. Plant Cell 7, 1347-1356.

Proveniers, M., Rutjens, B., Brand, M., and Smeekens, S. (2007) The Arabidopsis TALE homeobox gene ATH1 controls floral competency through positive regulation of FLC. Plant J. 52, 899-913.

Ragni, L., Belles-Boix, E., Gunl, M., and Pautot, V. (2008). Interactions of KNAT6 and KNAT2 with BREVIDPEDICELLUS and PENNYWISE in Arabidopsis inflorecences. Plant Cell 20, 888-900.

Rast, M.I., and Simon, R. (2008) The meristem-to-organ boundary: more than an extremity of anything. Curr. Opin. Genet. Dev. 18, 287-294. 
Ratcliffe, O.J., Amaya, I., Vincent, C.A., Rothstein, S., Carpenter, R., Coen, E.S., and Bradley, D. (1998) A common mechanism controls the life cycle and architecture of plants. Development 125, 1609-1615.

Reinhardt, D., and Kuhlemeier, C. (2002) Plant architecture. EMBO Rep. 3, 846-851.

Reinhardt, D., Pesce, E.-R., Stieger, P., Mandel, T., Baltensperger, K., Bennett, M., Traas, J., Friml, J., and Kuhlemeier, C. (2003) Regulation of phyllotaxis by polar auxin transport. Nature 426, 255-260.

Riechmann, J.L., Krizek, B.A., and Meyerowitz, E.M. (1996) Dimerization specificity of Arabidopsis MADS domain homeotic proteins APETALA1, APETALA3, PISTILLATA, and AGAMOUS. Proc. Natl. Acad. Sci. U S A. 93, 4793-4798.

Rochon, A., Boyle, P., Wignes, T., Fobert, P.R. and Despres, C. (2006) The coactivator function of Arabidopsis NPR1 requires the core of its BTB/POZ domain and the oxidation of C-terminal cysteines. Plant Cell 18, 3670-3685.

Ruiz-Garcia, L., Madueno, F., Wilkinson, M., Haughn, G., Salinas, J., and MartinezZapater, J.M. (1997) Different roles of flowering-time genes in the activation of floral initiation genes in Arabidopsis. Plant Cell 9, 1921-1934.

Rupp, H.M., Frank, M., Werner, T., Strnad, M., and Schmulling, T. (1999) Increased steady state mRNA levels of the STM and KNATI homeobox genes in cytokinin overproducing Arabidopsis thaliana indicate a role for cytokinins in the shoot apical meristem. Plant J. 18, 557-563.

Rutjens, B., Bao, D., van Eck-Stouten, E., Brand, M., Smeekens, S., and Proveniers, M. (2009) Shoot apical meristem function in Arabidopsis requires the combined activities of three BEL1-like homeodomain proteins. Plant J. 58, 641-654.

Sablowski, R. (2007) Flowering and determinacy in Arabidopsis. J. Exp. Bot. 58, 899-907.

Sablowski, R.W.M., and Meyerowitz, E.M. (1998) A homolog of NO APICAL MERISTEM is an immediate target of the floral homeotic genes APETALA3/PISTILLATA. Cell 92, 93103.

Saddic, L.A., Huvermann, B., Bezhani, S., Su, Y., Winter, C. M., Kwon, C.S., Collum, R.P., and Wagner, D. (2006) The LEAFY target LMI1 is a meristem identity regulator and acts together with LEAFY to regulate expression of CAULIFLOWER. Development 133, 16731682.

Saleh, H., Alvarez-Venegas, R., and Avramova, Z. (2008) An efficient chromatin immunoprecipitation (ChIP) protocol for studying histone modifications in Arabidopsis plants. Nat. Protoc. 3, 1018-25.

Saleh, O., Issman, N., Seumel, G.I., Stav,R., Samach, A., Reski, R., Frank, W., and Arazi, T. (2011) MicroRNA534a control of BLADE-ON-PETIOLE1 and 2 mediates juvenile-toadult gametophyte transition in Physcomitrella patens. Plant J. 65, 661-674.

Samach, A., Onouchi, H., Gold, S.E., Ditta, G.S., Schwartz-Sommer, Z., Yanofsky, M.F., and Coupland, G. (2000) Distinct roles of CONSTANS target genes in reproductive development of Arabidopsis. Science 288, 1613-1616. 
Schoof, H., Lenhard, M., Haecker, A., Mayer, K.F., Jürgens, G., and Laux, T. (2000) The stem cell population of Arabidopsis shoot apical meristems is maintained by a regulatory loop between the CLAVATA and WUSCHEL genes. Cell 100, 635-644.

Schultz, E.A., and Haughn G.W. (1991) LEAFY, a homeotic gene that regulates inflorescence development in Arabidopsis. Plant Cell 3, 771-781.

Schultz, E.A., and Haughn, G.W. (1993) Genetic analysis of the floral initiation process (FLIP) in Arabidopsis. Development 119, 745-765.

Schwechheimer, C., and Willige, B.C. (2009) Shedding light on gibberellic acid signaling. Curr. Opin. Plant Biol. 12, 57-62.

Semiarti, E., Ueno, Y., Tsukaya, H., Iwakawa, H., Machida, C., and Machida, Y. (2001) The ASYMMETRIC LEAVES2 gene of Arabidopsis thaliana regulates formation of a symmetric lamina, establishment of venation and repression of meristem-related homeobox genes in leaves. Development 128, 1771-1783.

Sessions, A., Yanofsky, M.F. and Weigel, D. (2000) Cell-cell signaling and movement by the floral transcription factors LEAFY and APETALA1. Science 289, 779-781.

Shani, E., Ben-Gera, H., Shleizer-Burko, S., Burko, Y., Weiss, D., and Ori N. (2010) Cytokinin regulates compound leaf development in tomato. Plant Cell 22, 3206-3217.

Shannon, S., and Meeks-Wagner, D.R. (1993) Genetic interactions that regulate inflorescence development in Arabidopsis. Plant Cell 5, 639-655.

Shuai, B., Reynaga-Pena, C. G., and Springer, P. S. (2002) The Lateral Organ Boundaries gene defines a novel, plant-specific gene family. Plant Physiol. 129, 747-761.

Sieburth, L.E. (1999). Auxin is required for leaf vein pattern in Arabidopsis. Plant Physiol. 121, 1179-1190.

Sieburth, L.E., and Meyerowitz, E.M. (1997) Molecular dissection of the AGAMOUS control region shows that cis elements for spatial regulation are located intragenically. Plant Cell 9 , 355-365.

Skoog, F., and Miller, C.O. (1957) Chemical regulation of growth and organ formation in plant tissue cultured in vitro. Symp. Soc. Exp. Biol XI, 118-131.

Smith, H.M.S., and Hake, S. (2003) The interaction of two homeobox genes, BREVIPEDICELLUS and PENNYWISE, regulates internode patterning in the Arabidopsis inflorescence. Plant Cell 15, 1717-1727.

Smith, H.M.S., Campbell, B.C., and Hake, S. (2004) Competence to respond to floral inductive signals requires the homeobox genes PENNYWISE and POUND-FOOLISH. Curr. Biol. 14, 812-817.

Smyth, D.R., Bowman, J.L., and Meyerowitz, E.M. (1990) Early flower development in Arabidopsis. Plant Cell 2, 755-767.

Song, Y.H., Song, N.Y., Shin, S.Y., Kim, H.J., Yun, D.J., Lim, C.O., Lee, S.Y., Kang, K.Y., and Hong, J.C. (2008) Isolation of CONSTANS as a TGA4/OBF4 interacting protein. Mol. Cells 25, 559-656. 
Spoel, S.H., Mou, Z., Tada, Y., Spivey, N,W., Genschik, P., and Dong, X. (2009) Proteasomemediated turnover of the transcription coactivator NPR1 plays dual roles in regulating plant immunity. Cell 137, 860-872.

Spoel, S.H., Tada, Y., and Loake, G.J. (2010) Post-translational protein modification as a tool for transcription reprogramming. New Phytol. 186, 333-339.

Sundstrom, J.F., Nakayama, N., Glimelius, K., and Irish, V.F. (2006) Direct regulation of the floral homeotic APETALA1 gene by APETALA3 and PISTILLATA in Arabidopsis. Plant $J$. 46, 593-600.

Sussex, I.M. (1989). Developmental programming of the shoot meristem. Cell 56, 225-229.

Tada, Y., Spoel, S. H., Pajerowska-Mukhtar, K., Mou, Z., Song, J., Wang, C., Zuo, J., and Dong, X. (2008) Plant immunity requires conformational charges of NPR1 via Snitrosylation and thioredoxins. Science 321, 952-956.

Takada, S., Hibara, K.-I., Ishida, T., and Tasaka, M. (2001) The CUP-SHAPED COTYLEDON1 gene of Arabidopsis regulates shoot apical meristem formation. Development 128, 1127-1135.

Taylor, S., Hofer, J., and Murfet, I. (2001) Stamina pistilloida, the pea ortholog of Fim and $\mathrm{UFO}$, is required for normal development of flowers, inflorescences, and leaves. Plant Cell 13, 31-46.

Turck, F., Fornara, F., and Coupland, G. (2008) Regulation and identity of florigen: FLOWERING LOCUS T moves to center stage. Annu. Rev. Plant Biol. 59, 573-594.

Uchida, N., Kimura, S., Koenig, D., and Sinha, N. (2010) Coordination of leaf development via regulation of KNOX1 genes. J. Plant Res. 123, 7-14.

Ung, N., Lal, S., and Smith, H. (2011) The role of PENNYWISE and POUND-FOOLISH in the maintenance of the shoot apical meristem in Arabidopsis. Plant Physiol. 156, 605-614.

Venglat, S.P., Dumonceaux, T., Rozwadowski, K., Parnell, L., Babic, V., Keller, W., Martienssen, R., Selvaraj, G., and Datla, R. (2002) The homeobox gene BREVIPEDICELLUS is a key regulator of inflorescence architecture in Arabidopsis. Proc. Natl. Acad. Sci. USA 99, 4730-4735.

Vernoux, T., Besnard, F., and Traas, J. (2010) Auxin at the shoot apical meristem. Cold Spring Harb. Perspect. Biol. 2, a001287.

Vernoux, T., Kronenberger, J., Grandjean, O., Laufs, P., and Traas, J. (2000) PINFORMED1 regulates cell fate at the periphery of the shoot apical meristem. Development 127, 5157-5165.

Vroemen, C. W., Mordhorst, A.P., Albrecht, C., Kwaaitaal, M.A.C.J., and de Vries, S.C. (2003) The CUP-SHAPED COTYLEDON3 gene is required for boundary and shoot meristem formation in Arabidopsis. Plant Cell 15, 1563-1577.

Wagner, D., Sablowski, R.W.M., and Meyerowitz, E.M. (1999) Transcriptional activation of APETALA1 by LEAFY. Science 285, 582-584.

Wang, J.W., Czech, B., and Weigel, D. (2009) miR156-regulated SPL transcription factors define an endogenous flowering pathway in Arabidopsis thaliana. Cell 138, 738-749. 
Weigel, D. (1995) The genetics of flower development: from floral induction to ovule morphogenesis. Annu. Rev. Genet. 29, 19-39.

Weigel, D. (1998) From floral induction to floral shape. Curr. Opin. Plant Biol. 1, 55-59.

Weigel, D., and Meyerowitz, E.M. (1993) Activation of floral homeotic genes in Arabidopsis. Science 261, 1723-1726.

Weigel, D., and Nilsson, O. (1995) A developmental switch sufficient for flower initiation in diverse plants. Nature 377, 495-500.

Weigel, D., Alvarez, J., Smyth, D.R., Yanofsky, M.F., and Meyerowitz, E.M. (1992) LEAFY controls floral meristem identity in Arabidopsis. Cell 69, 843-859.

Wigge, P.A., Kim, M.C., Jaegar, K.E., Busch, W., Schmid, M., Lohmann, J.U., and Weigel, D. (2005) Integration of spatial and temporal information during floral induction in Arabidopsis. Science 309, 1056-1059.

Wilkinson, M.D., and Haughn, G.W. (1995) UNUSUAL FLORAL ORGANS controls meristem identity and organ primordia fate in Arabidopsis. Plant Cell 7, 1485-1499.

William, D.A., Su, Y., Smith, M.R., Lu, M., Baldwin, D.A., and Wagner, D. (2004) Genomic identification of direct targets genes of LEAFY. Proc. Natl. Acad. Sci. USA 101, 1775-1780.

William, L., and Fletcher, J.C. (2005) Stem cell regulation in the Arabidopsis shoot apical meristem. Curr.Opion. Plant Biol. 8, 582-586.

Wollmann, H., Mica, E., Todesco, M., Long, J.A. and Weigel, D. (2010) On reconciling the interactions between APETALA2, miR172 and AGAMOUS with the ABC model of flower development. Development 137, 3633-3642.

Wu, G., Park, M.K., Conway, S.R., Wang, J.W., Weigel, D., and Poethig, R.S. (2009) The sequential action of miR156 and miR172 regulates developmental timing in Arabidopsis. Cell 138, 750-759.

Xing, S., Rosso, M.G., and Zachgo, S. (2005) ROXY1, a member of the plant glutaredoxin family, is required for petal development in Arabidopsis thaliana. Development 132, 15551565.

Xu, B., Li, Z., Zhu, Y., Wang, H., Ma, H., Dong, A., and Huang, H. (2008) Arabidopsis genes AS1, AS2, and JAG negatively regulate boundary-specifying genes to promote sepal and petal development. Plant Physiol. 146, 566-575.

Xu, L., Xu, Y., Dong, A., Sun, Y., Pi, L., Xu, Y., and Huang, H. (2003) Novel as1 and as2 defects in leaf adaxial-abaxial polarity reveal the requirement for ASYMMETRIC LEAVESI and 2 and ERECTA functions in specifying leaf adaxial identity. Development 130, 40974107.

Xu, M., Hu, T., McKim, S., Murmu, J., Haughn, G.W., and Hepworth, S.R. (2010) Arabidopsis BLADE-ON-PETIOLE1 and 2 promote floral meristem fate and determinacy in a previously undefined pathway targeting APETALA1 and AGAMOUS-LIKE24. Plant $J$. 63, 974-989. 
Xu, Y.Y., Wang, X.M., Li, J., Li, J.H., Wu, J.S., Walker, J.C., Xu, Z.H., and Chong, K. (2005) Activation of the WUS gene induces ectopic initiation of floral meristems on mature stem surface in Arabidopsis thaliana. Plant Mol. Biol. 58, 773-784.

Yamaguchi, A., Wu, M.-F., Yang, L., Wu, G., Poethig, R.S., and Wagner, D. (2009) The microRNA-regulated SBP-box transcription factor SPL3 is a direct upstream activator of LEAFY, FRUITFULL, and APETALA1. Dev. Cell 17, 268-278.

Yanai, O., Shani, E., Dolezal, K., Tarkowski, P., Sablowski, R., Sandberg, G., Samach, A. ,and Ori, N. (2005) Arabidopsis KNOX1 proteins activate cytokinin biosynthesis. Curr. Biol. 15, 1566-1571.

Yoo, S.D., Cho, Y.H., and Sheen, J. (2007) Arabidopsis mesophyll protoplasts: a versatile cell system for transient gene expression analysis. Nat. Protoc. 2, 1565-72.

Yu, H., Ito, T., Wellmer, F., and Meyerowitz, E.M. (2004) Repression of AGAMOUS-LIKE 24 is a crucial step in promoting flower development. Nat. Genet. 36, 157-161.

Yu, H., Xu, F., Tan, E.L., and Kumar, P.P. (2002) AGAMOUS-LIKE 24, a dosage-dependent mediator of the flowering signals. Proc. Natl. Acad. Sci. USA 99, 16336-16341.

Yu, L., Patibanda, V., and Smith, H.M. (2009) A novel role of BELL1-like homeobox genes, PENNYWISE and POUND-FOOLISH, in floral patterning. Planta 229, 693-707.

Zhang, Y., Fan, W., Kinkema, M., Li, X., and Dong, X. (1999) Interaction of NPR1 with basic leucine zipper protein transcription factors that bind sequences required for salicylic acid induction of the PR-1 gene. Proc. Natl. Acad. Sci. USA 96, 6523-6528. 


\section{Appendix A. Summary of regulatory genes related to this thesis}

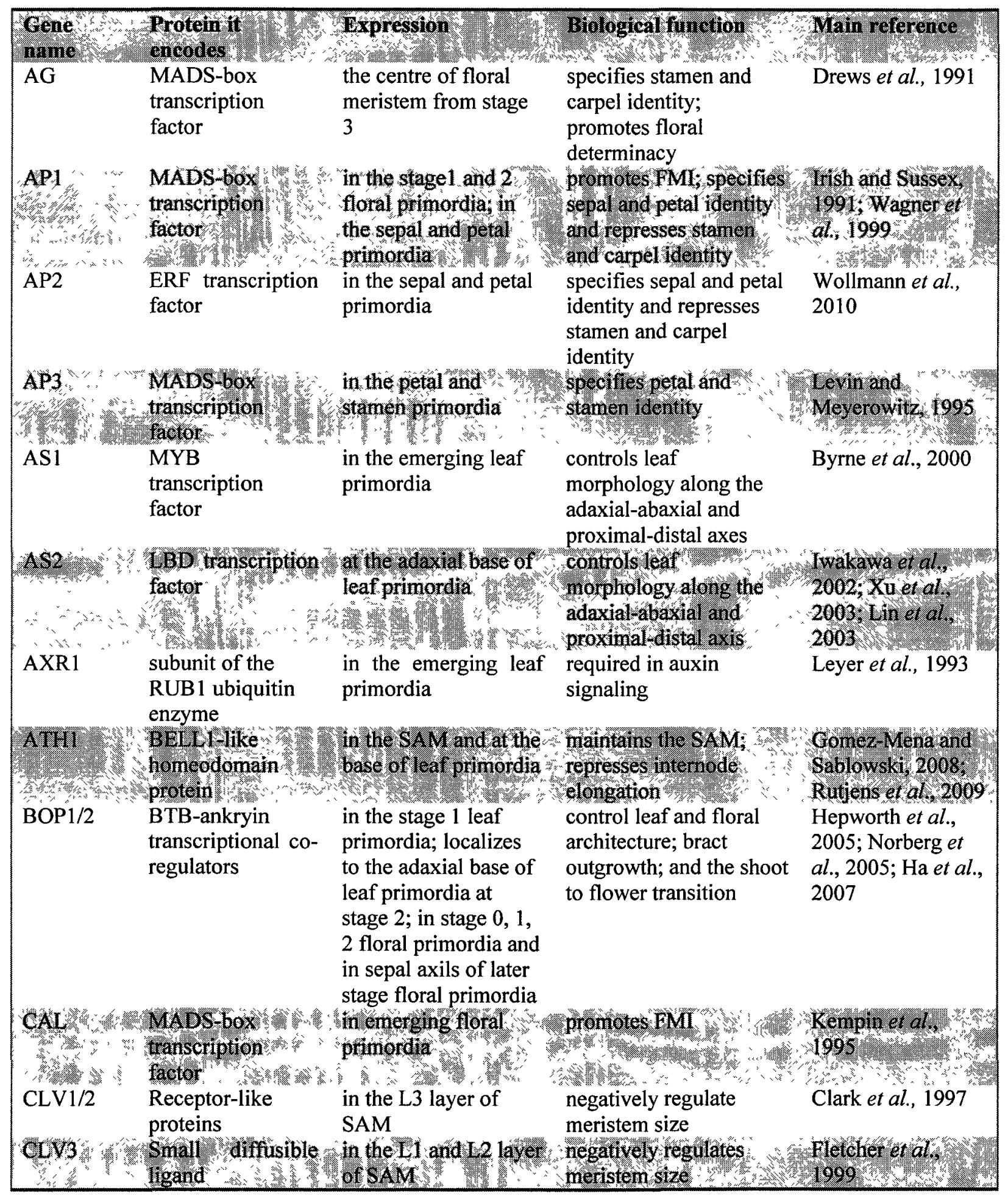




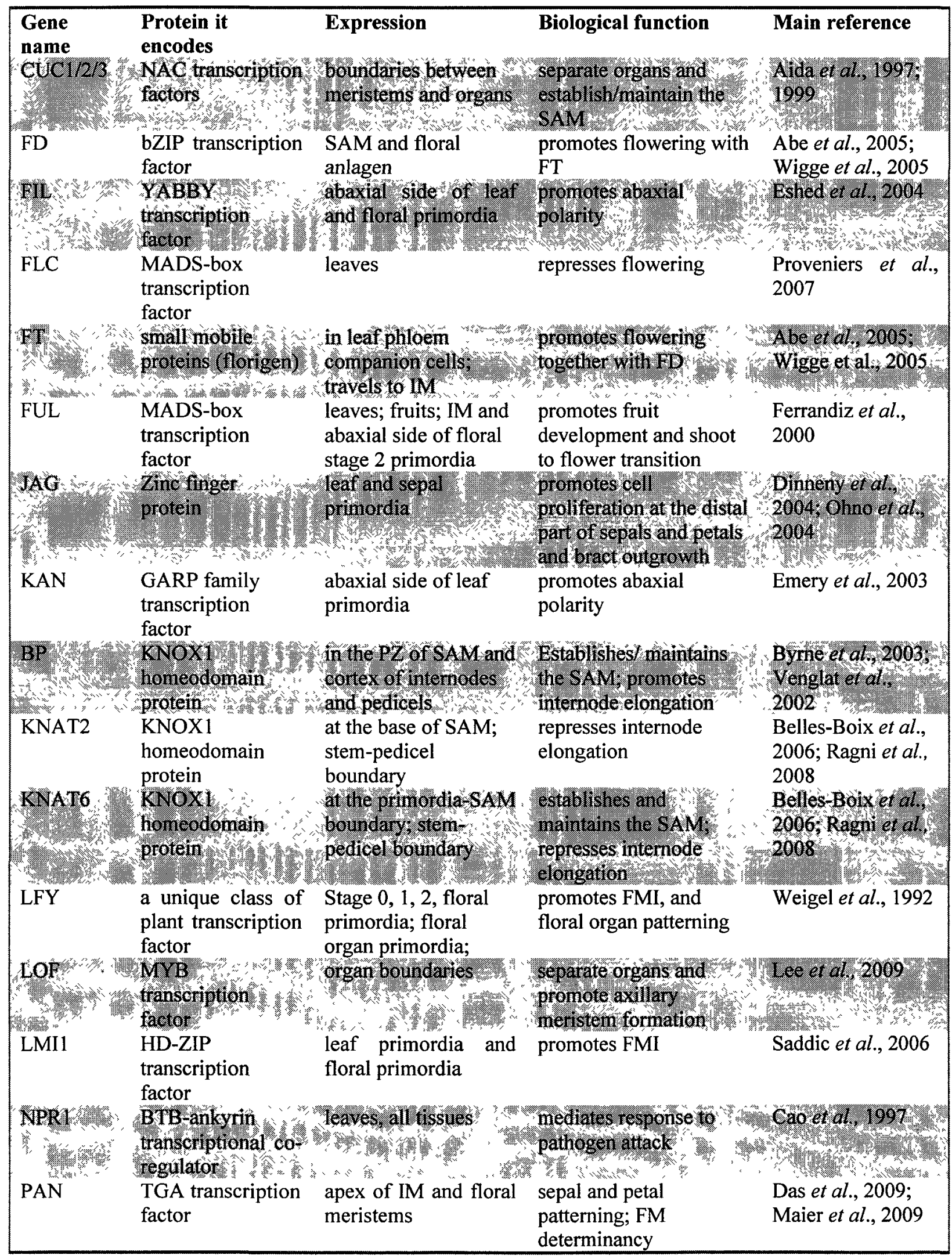




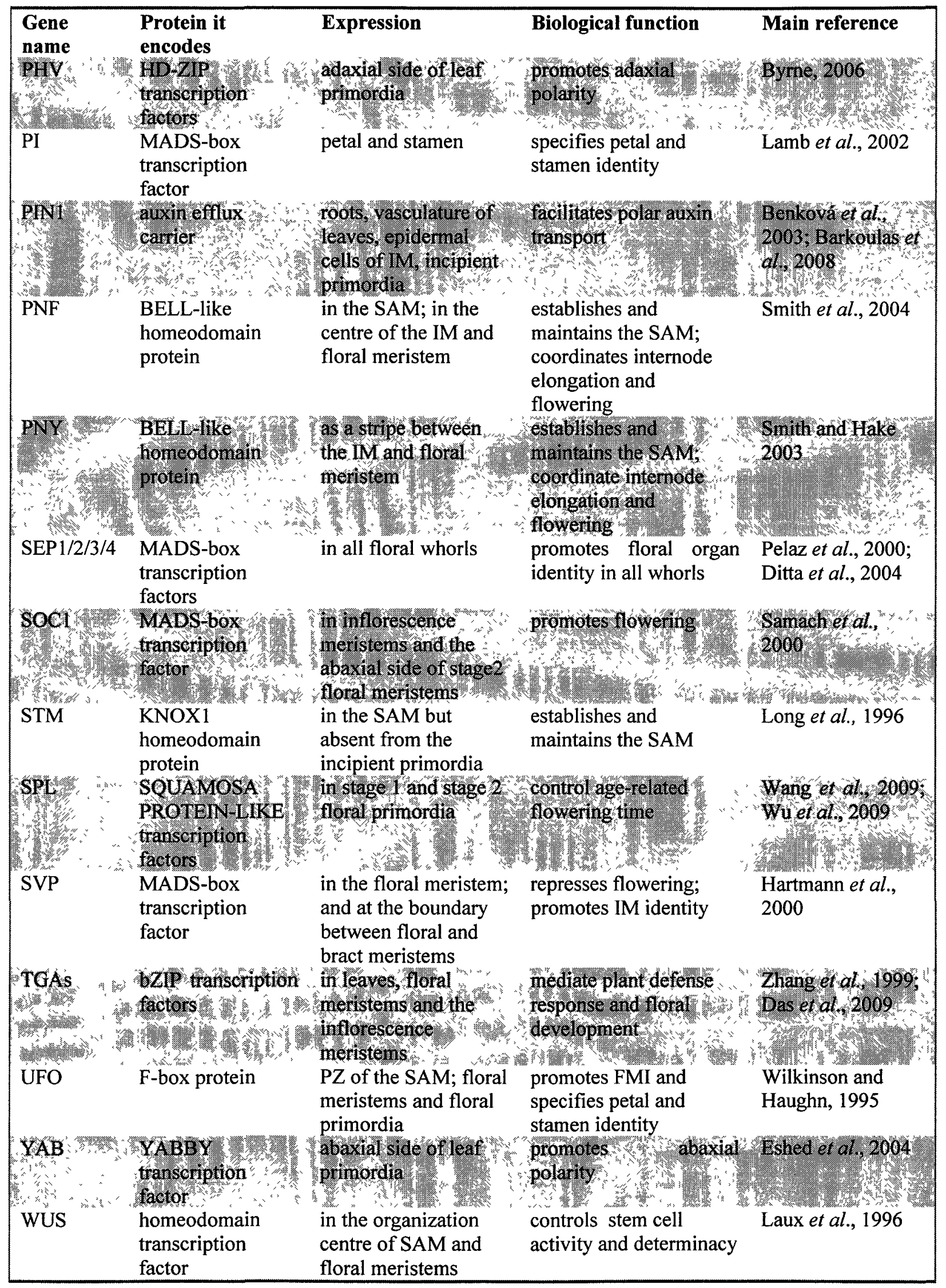




\section{Appendix B. Genetic interactions in double mutant analysis and what they mean}

Analysis of double mutant phenotypes is employed extensively as a major guide line to determine the potential mode of interaction between two genes (for example A and B). This method is especially powerful because it can give answers without knowledge of all of the genes in a pathway or the products that they encode. Interactions may be described as additive, synergistic, antagonistic, or epistatic. Criteria for these interactions are described below.

If the phenotype of the double mutant is additive (a plus b), it indicates that the two genes being analyzed function in non-interacting pathways. However, if the phenotype of the double mutant is synergistic (more than a plus b, a new phenotype may occur), it indicates that the two genes function in redundant pathways. This redundancy may be partial or complete. If A can completely compensate for $\mathrm{B}$ then gene function is completely redundant. In many cases however, two genes are only partially redundant since they may share a subset of developmental or cellular functions but they also each have unique functions in other processes. Closely related members of a gene family often have partial redundancy in which a shared set of functions are preserved and independent functions might also be involved (Pickett and Meeks-Wagner, 1995). When synergy occurs between closely related members of a gene family, it is often the case that the genes function together in one pathway, sharing the same task in a homologous fashion. Functional redundancy can also occur between non-homologous genes or pathways. This is often true of developmental processes, such as flowering, which are governed by multiple regulatory pathways. In this case, synergy arises when two pathways that converge at a node are disrupted (Pérez-Pérez et al. 2009).

When a double mutant is made, phenotypes can be enhanced (worsened) or suppressed (improved). If mutation of gene $\mathrm{B}$ enhances the phenotype of $\mathrm{A}$, then both are functioning redundantly to control a phenotypic trait as discussed above. If on the other hand, mutation of gene $\mathrm{B}$ suppresses the phenotype of $\mathrm{A}$, then genes $\mathrm{A}$ and $\mathrm{B}$ function antagonistically. Antagonistic interactions may be observed for example if gene $\mathrm{B}$ is misexpressed in mutant $\mathrm{A}$, or when genes $\mathrm{A}$ and $\mathrm{B}$ might have opposite effects on the transcription of gene $\mathrm{C}$, which is a master regulator of the phenotypic trait in question. The reason for the antagonistic genetic interaction must be evaluated empirically. 
Double mutant analysis may also give information about the order of gene function in a linear pathway. This is most easily assayed when single mutations ( $a$ and $b$ ) produce different phenotypes from the wild-type and from each other, and the double mutant phenotype looks like one of the phenotypes produced by a single mutation ( $a$ or $b$ ). This mutation is said to be epistatic to the other. The epistatic mutation may be in either the upstream or downstream gene, depending on the nature of the two mutations and the type of regulation. In a simple linear pathway in which the regulatory interactions are all positive, the upstream mutation will be epistatic to the downstream mutation. (Avery and Wasserman, 1992). 